\title{
ASSESSMENT OF ASYMMETRIC FLIGHT ON SOLAR UAS
}

\author{
A Thesis \\ presented to \\ the Faculty of California Polytechnic State University, \\ San Luis Obispo
}

\author{
In Partial Fulfillment \\ of the Requirements for the Degree \\ Master of Science in Aerospace Engineering
}

by

Eric Belfield

December 2016 
(C) 2016

Eric Belfield

ALL RIGHTS RESERVED 


\section{COMMITTEE MEMBERSHIP}

TITLE:

Assessment of Asymmetric Flight on Solar UAS

AUTHOR:

Eric Belfield

DATE SUBMITTED: December 2016

COMMITTEE CHAIR: Aaron Drake, Ph.D.

Associate Professor

Aerospace Engineering Department

COMMITTEE MEMBER: Graham Doig, Ph.D.

Assistant Professor

Aerospace Engineering Department

COMMITTEE MEMBER: Robert McDonald, Ph.D.

Professor

Aerospace Engineering Department

COMMITTEE MEMBER: Marc Horney, Ph.D.

Assistant Professor

Animal Science Department 


\section{ABSTRACT \\ Assessment of Asymmetric Flight on Solar UAS}

Eric Belfield

An investigation was conducted into the feasibility of using an unconventional flight technique, asymmetric flight, to improve overall efficiency of solar aircraft. In this study, asymmetric flight is defined as steady level flight in a non-wings-level state intended to improve solar incidence angle. By manipulating aircraft orientation through roll angle, solar energy collection is improved but aerodynamic efficiency is worsened due to the introduction of additional trim drag. A point performance model was developed to investigate the trade-off between improvement in solar energy collection and additional drag associated with asymmetric flight. A mission model with a focus on aircraft orbits was then developed via integration of the point performance model over a set of discrete points.

It is shown that there is a non-zero bank angle where optimal net power is achieved for a given aircraft orientation, flight condition, and sun position. The study also shows that there is improvement in overall efficiency over conventional flight for various orbit shapes and winds aloft. This indicates that there is potential value in not only flight path planning, but also in orientation planning for solar aircraft. 


\section{ACKNOWLEDGMENTS}

Thanks to my parents for their encouragement and support in letting me set my own goals. My grandfather, for the inspiration to go for a master's degree. To Dr. Aaron Drake, for his guidance and, at times, wisdom. Finally, to Andrew, Mike, and

the rest of the Autonomous Flight Laboratory for their support during my studies at Cal Poly. 


\section{TABLE OF CONTENTS}

Page

LIST OF TABLES . . . . . . . . . . . . . . . . . viii

LIST OF FIGURES . . . . . . . . . . . . . . . . . . CHAPTER

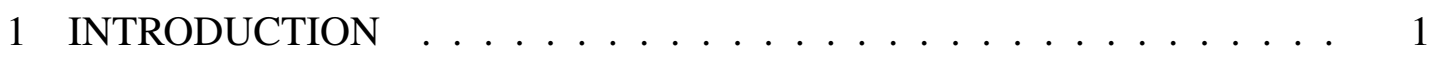

1.1 Background .......................... 1

1.2 Focus on Small UAS . . . . . . . . . . . . . . . 2

1.3 Solar-Photovoltaic Energy Collection . . . . . . . . . . 4

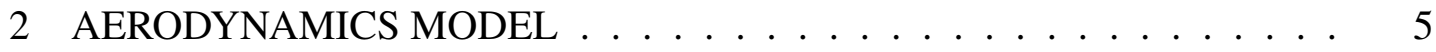

2.1 Balance of Forces . . . . . . . . . . . . . . 5

2.2 Modeling Lift and Longitudinal Stability . . . . . . . . . . . . . 7

2.2.1 Vortex Lattice for the Main Wing and Stabilator . . . . . . . 8

2.2.2 The Cross-Flow Principle . . . . . . . . . . . . . 10

2.2.3 Calculation of Longitudinal Trim . . . . . . . . . . . 11

2.3 Modeling Side Force and Directional Stability . . . . . . . . . . . . 13

2.3.1 Calculation of Forces in the Yaw Axis . . . . . . . . . . 14

2.3.2 Trim in the Yaw Axis . . . . . . . . . . . . 15

2.4 Correlating Bank Angle and Sideslip Angle . . . . . . . . . . . . . 18

2.5 Parasite Drag . . . . . . . . . . . . . . . . 18

2.6 Asymmetric Flight Drag Polar . . . . . . . . . . . . . . 20

2.7 Aerodynamics Model Validation . . . . . . . . . . . . . 20

2.8 Calculation of Power Required . . . . . . . . . . . . . . . 25

3 SOLAR-PHOTOVOLTAIC MODEL . . . . . . . . . . . . . 27

3.1 Solar-Electric Propulsion Model . . . . . . . . . . . . . . . . 27

3.2 Sun Angles . . . . . . . . . . . . . . . . . . . 28

3.3 Solar Irradiance . . . . . . . . . . . . . . . . . . . 30

3.3.1 Alternate Model for Atmospheric Transmittance . . . . . . 32

3.4 Incidence Angle . . . . . . . . . . . . . . . . . . . 34 
3.5 Solar-Photovoltaic Model Validation . . . . . . . . . . . . . 38

4 POINT PERFORMANCE RESULTS . . . . . . . . . . . . . . . . . . 41

4.1 Constant-Heading Results . . . . . . . . . . . . . . . . . 41

4.2 Steady Turn Results . . . . . . . . . . . . . . . . . . 50

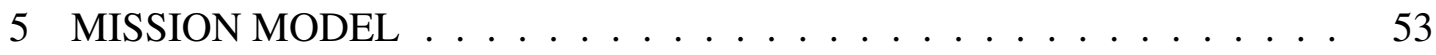

5.1 Definition of Aircraft Track . . . . . . . . . . . . . . . . 53

5.2 Adding Winds Aloft . . . . . . . . . . . . . . . 57

6 MISSION RESULTS . . . . . . . . . . . . . . . . . . . . 62

6.1 Circular Orbit Performance . . . . . . . . . . . . . . . . . 63

6.1 .1 Winds on Circular Orbits . . . . . . . . . . . . . 68

6.2 Elliptical Orbit Performance . . . . . . . . . . . . . . 70

6.2 .1 Winds on Elliptical Orbits _ . . . . . . . . . . . 73

6.3 Grid Flight Performance . . . . . . . . . . . . . . . 76

6.3.1 Winds on Grid Flight . . . . . . . . . . . . . . 80

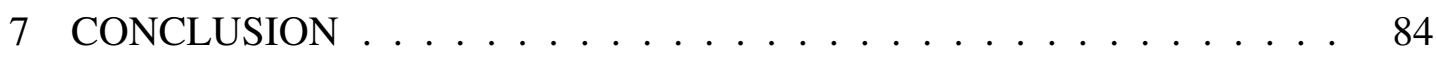

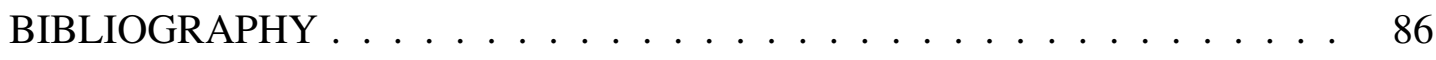
APPENDICES

A TIME HISTORIES OF SELECTED MISSIONS $\ldots \ldots \ldots \ldots$

B MATLAB CODE ..................... 95 


\section{LIST OF TABLES}

Table

Page

2.1 Calculated Parasite Drag Coefficient for the Puma AE. . . . . . . . 19

2.2 Comparison of lift values for several CFD mesh resolutions. . . . . 23

2.3 Comparison of lift and drag coefficients between analytical and CFD models. ........................ 24

2.4 Comparison of pitching moment coefficient between analytical and CFD models. . . . . . . . . . . . . . . . . . . 25

3.1 Assumed Puma AE energy conversion efficiencies . . . . . . . . 28

4.1 Net power for asymmetric and conventional flight techniques flying north on the summer solstice in San Luis Obispo, CA. . . . . . . .

6.1 Comparison of Net Energy for two different orbit radii throughout the summer solstice in San Luis Obispo, CA. . . . . . . . . . . .

6.2 Comparison of net energy for a circular orbit of radius $400 \mathrm{ft}$, with varying wind directions at $5 \mathrm{kt} . \ldots \ldots \ldots$

6.3 Comparison of Net Energy for different orbit offsets throughout the summer solstice in San Luis Obispo, CA with the sun placed in the east. . . . . . . . . . . . . . . .

6.4 Comparison of net energy for an elliptical orbit, varying orbit offset, with wind at $360^{\circ}, 5 \mathrm{kt}$ and the sun in the east at elevation 45 degrees. 74

6.5 Comparison of net energy for an elliptical orbit, varying orbit offset, with wind at $180^{\circ}, 5 \mathrm{kt}$ and the sun in the east at elevation 45 degrees. 75

6.6 Comparison of net energy for an elliptical orbit, varying orbit offset, with wind at $045^{\circ}, 5 \mathrm{kt}$ and the sun in the east at elevation 45 degrees. 76

6.7 Comparison of Net Energy for different grid offsets throughout the summer solstice in San Luis Obispo, CA with the sun placed in the east. . . . . . . . . . . . . . . . .

6.8 Comparison of net energy for a grid pattern, varying grid offset, with wind at $360^{\circ}, 5 \mathrm{kt}$ and the sun in the east at elevation 45 degrees. . .

6.9 Comparison of net energy for a grid pattern, varying grid offset, with wind at $090^{\circ}, 5 \mathrm{kt}$ and the sun in the east at elevation 45 degrees. . . 
6.10 Comparison of net energy for a grid pattern, varying grid offset, with wind at $090^{\circ}, 5 \mathrm{kt}$ and the sun in the west at elevation 45 degrees. 


\section{LIST OF FIGURES}

Figure Page

1.13 -view of AeroVironment Puma AE. . . . . . . . . . . 3

1.2 Characteristics of a photovoltaic solar cell in 2-D. . . . . . . . . 4

$2.1 \quad$ Forces acting on an aircraft in asymmetric flight. . . . . . . 6

$2.2 \quad$ Forces considered for longitudinal stability. . . . . . . . . . . 8

2.3 Lift curve for the Puma's main wing only. . . . . . . . . . . . 9

2.4 The cross-flow principle applied to the fuselage of the Puma AE. . . 10

2.5 Free-body-diagram of longitudinal trim analysis. . . . . . . . 13

2.6 Trimmed lift curve. . . . . . . . . . . . . . . . . . . . . . . 14

$2.7 \quad$ Free-body-diagram of directional trim analysis. . . . . . . . . . 15

2.8 Free-body-diagram of directional trim analysis. . . . . . . . . . 16

2.9 Trimmed side force curve. . . . . . . . . . . . . . 17

2.10 Simplified AeroVironment RQ-20 Puma AE geometry model. . . . 19

2.11 Asymmetric flight drag polar. . . . . . . . . . . . 21

2.12 Locations of fluid boundaries in CFD simulation. . . . . . . . . 22

2.13 Contours of static pressure in Puma AE CFD simulation. . . . . . . 24

3.1 The Earth-fixed NED and aircraft body coordinate system. . . . . . 37

3.2 Experimental apparatus for the solar-photovoltaic model ground test. 38

3.3 Irradiance vs. Time of Day for the solar sensor ground test performed 2 Nov. 2016, comparing experimental and alternate model data. . . . . . . . . . . . . . . . . 39

3.4 Irradiance vs. Time of Day, comparing primary and alternate model data. . . . . . . . . . . . . . . . . .

4.1 Net power for the Puma AE flying in San Luis Obispo, CA on the summer solstice at 8:00 AM (left) and 12:00 PM (right). . . . . .

4.2 Net power for the Puma AE flying in San Luis Obispo, CA on the summer solstice at 12:00 PM (left) and 4:00 PM (right). . . . . . 
4.3 Bank angle for the Puma AE flying north asymmetrically in San Luis Obispo, CA through daytime on the summer solstice. . . . . . . . . 44

4.4 Net power for the Puma AE flying asymmetrically in San Luis Obispo, CA throughout an entire year for 3 different times of day. . . . . . .

4.5 Net power for the Puma AE flying north in San Luis Obispo, CA on the summer solstice, both conventionally and asymmetrically. . . .

4.6 Net power for the Puma AE flying asymmetrically at San Luis Obispo's longitude on the northern hemisphere's summer solstice at varying latitudes. . . . . . . . . . . . . . . . . .

4.7 Geographic locations of the test points shown in Fig. 4.6. The selected longitude is that of San Luis Obispo, CA: $120.6^{\circ} \mathrm{W}$. . . . . .

4.8 Net and required power for the Puma AE flying asymmetrically in San Luis Obispo, CA at noon on the summer solstice for various turn radii. . . . . . . . . . . . . . .

4.9 Net and required power for the Puma AE flying asymmetrically in San Luis Obispo, CA at 4:00 PM on the summer solstice for various turn radii. . . . . . . . . . . . . . . . .

5.1 Visualization of bearing and distance for a sample mission leg between points 1 and $2 \ldots \ldots \ldots \ldots \ldots \ldots$. . . . . . . . 54

5.2 Mission case where simple subtraction of heading angles is insuffi-

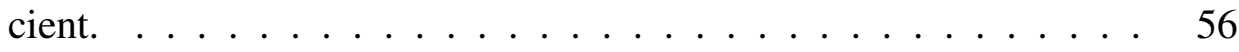

5.3 Example case showing vectors used to model effects of winds aloft. 58

5.4 Effects of a 5-knot wind from 270 degrees on ground speed for a circular orbit. . . . . . . . . . . . . . .

5.5 Effects of a 10-knot wind from 180 degrees on ground speed for a circular orbit. . . . . . . . . . . . . .

6.1 Net Energy vs. Orbit Radius for various times of day on the summer solstice in San Luis Obispo, CA. . . . . . . . . . . . . . . .

6.2 Net Energy vs. Orbit Radius for various times of day on the winter solstice in San Luis Obispo, CA. . . . . . . . . . . . . . . . . 64

6.3 Average Power vs. Orbit Radius for asymmetric flight over various solar elevation angles on the summer solstice in San Luis Obispo, CA. 65

6.4 Average Power vs. Orbit Radius for asymmetric flight over various solar elevation angles on the winter solstice in San Luis Obispo, CA. 66 
6.5 Average Power vs. Orbit Radius for asymmetric flight over various solar elevation angles on the summer equinox in San Luis Obispo,

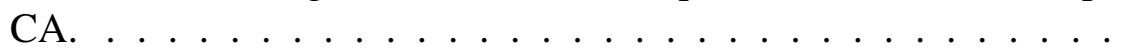

6.6 Example of path aircraft will fly through the air in order to maintain a circular ground track in the presence of winds as shown. . . . . 69

6.7 Illustration of terminology used to describe elliptical orbits. . . . . 70

6.8 Average Power for asymmetric flight with varying orbit offset throughout the day on the summer solstice in San Luis Obispo, CA. Azimuth angle is set to 90 degrees for all cases. . . . . . . . . . . . . . . 71

6.9 Average Power for asymmetric flight with varying orbit offset throughout the day on the winter solstice in San Luis Obispo, CA. Azimuth angle is set to 90 degrees for all cases. . . . . . . . . . . . . .

6.10 Example of path aircraft will fly through the air in order to maintain an elliptical ground track in the presence of winds as shown. . . . . 74

6.11 Illustration of the calculation of sensor swath width based on field of view angle. . . . . . . . . . . . . . 76

6.12 The first two segments of a land survey grid, used to assess grid mission performance. . . . . . . . . . . . . .

6.13 Average Power for asymmetric flight with varying grid offset throughout the day on the summer solstice in San Luis Obispo, CA. Azimuth angle is set to 90 degrees for all cases. . . . . . . . . . . .

6.14 Average Power for asymmetric flight with varying grid offset throughout the day on the winter solstice in San Luis Obispo, CA. Azimuth angle is set to 90 degrees for all cases. . . . . . . . . . .

6.15 Grid offset of $090^{\circ}$ under the effects of $5 \mathrm{kt}$ winds at $360^{\circ}$ with the sun in the east. . . . . . . . . . . . . . . . . .

A.1 Time history of bank angles for a sample circular orbit on the summer solstice in San Luis Obispo, CA. . . . . . . . . . . . . .

A.2 Time history of solar incidence angles for a sample circular orbit on the summer solstice in San Luis Obispo, CA. . . . . . . . . . . .

A.3 Time history of net power for a sample circular orbit on the summer solstice in San Luis Obispo, CA. . . . . . . . . . . . . . . . 91

A.4 Time history of bank angles for a sample elliptical orbit on the summer solstice in San Luis Obispo, CA. . . . . . . . . . . . . . 92

A.5 Time history of solar incidence angles for a sample elliptical orbit on the summer solstice in San Luis Obispo, CA. . . . . . . . . . 
A.6 Time history of net power for a sample elliptical orbit on the summer solstice in San Luis Obispo, CA. . . . . . . . . . . . . . . . . 93

A.7 Time history of bank angle for a sample grid flight on the summer solstice in San Luis Obispo, CA. . . . . . . . . . . . . . . . . 93

A.8 Time history of incidence angle for a sample grid flight on the summer solstice in San Luis Obispo, CA. . . . . . . . . . . . . . . . 94

A.9 Time history of net power for a sample grid flight on the summer solstice in San Luis Obispo, CA. . . . . . . . . . . . . . . . . . 94 


\section{Chapter 1}

\section{INTRODUCTION}

\subsection{Background}

With recent and continuing advancements in related technology, solar-powered aircraft show increased promise and potential. Solar power can be used with the goal of achieving permanent endurance through energy balance, or to simply augment the performance of more conventional electric aircraft. Much effort has already been expended, and efficiency gained, in research and development of solar-electric platforms and powertrains. As a result, opportunity now exists to take advantage of these unique platforms, and potentially gain efficiency, by using new mission planning techniques.

A large portion of the upward-facing surface area of the aircraft must be dedicated to solar cell area, and this typically means that a majority of the aircraft's aerodynamic surfaces will be covered. Solar energy collection is dependent largely upon the incidence angle of solar radiation on those solar cells, with best efficiency achieved at small incidence angles. This leads to a potential net benefit in performance to be achieved through a flight profile selected to improve solar incidence angle. Energyoptimal path planning for solar aircraft has been covered in literature, with most focus on steady, level, coordinated flight. Indeed, virtually all aircraft are conventionally trimmed for wings-level flight. In this condition, steady level flight can be achieved in a simple and aerodynamically efficient manner, and passenger comfort is highest. However, development and preponderance of the unmanned aerial system (UAS) leads to the possibility of not only flight path planning, but also aircraft orientation planning.

In this case, asymmetric flight is steady level flight in a non-wings-level state in- 
tended to improve solar incidence angle. While the aircraft's velocity vector still points in the desired flight direction, control surfaces are used to point a wing-mounted solar array towards the sun. While it is conceivable to use any and all of the aircraft's three axes to manipulate solar incidence angle, the primary parameter of interest in this study is roll angle. An aircraft can be trimmed for a non-zero roll angle by sideslipping, and any conventional aircraft can do so to different degrees. Additional aerodynamic drag is introduced when trimming the aircraft to maintain this uncoordinated flight condition, and will trade against any improvement in solar energy collection. While significant, the additional drag introduced via sideslip is likely to be lower than, for example, trying to increase angle of attack as a means of improving solar incidence angle. Because of this trade-off between increased drag and increased solar energy, a high-level assessment must be performed to determine if a net benefit can be achieved. Initial feasibility of the technique can be examined with a point performance analysis.

Unmanned aerial systems are commonly used as platforms for observing ground targets, an application in which the aircraft would orbit a notionally stationary point on the ground. Work in [1] has shown that solar aircraft can benefit by strategically selecting an orbit radius and corresponding bank angle. In this study, it is desired to develop an understanding of the potential of the asymmetric flight technique in a point performance sense, and then extend that understanding to determine if an asymmetric flight strategy can be used to further improve orbit or mission performance.

\subsection{Focus on Small UAS}

This work is focused on the small UAS (sUAS) class of aircraft, selected for their accessibility at the university level, common use of electric powertrains, which lends relevancy to their application as solar aircraft, and typical use as reconnaissance plat- 
forms where endurance is critical. The AeroVironment RQ-20 Puma AE was selected as a representative vehicle of the sUAS category. The Puma is a fixed-wing, 12-lb electric UAS with a 9.2-ft wingspan and capacity for a small modular payload. It is commonly used for target observation and reconnaissance missions, and cruises in the range of 20 to 40 knots at altitudes up to $10,500 \mathrm{ft}$ MSL. While the Puma is not a solar aircraft, its geometry and other parameters allow the modeling approach taken in this examination to be grounded by a real aircraft.

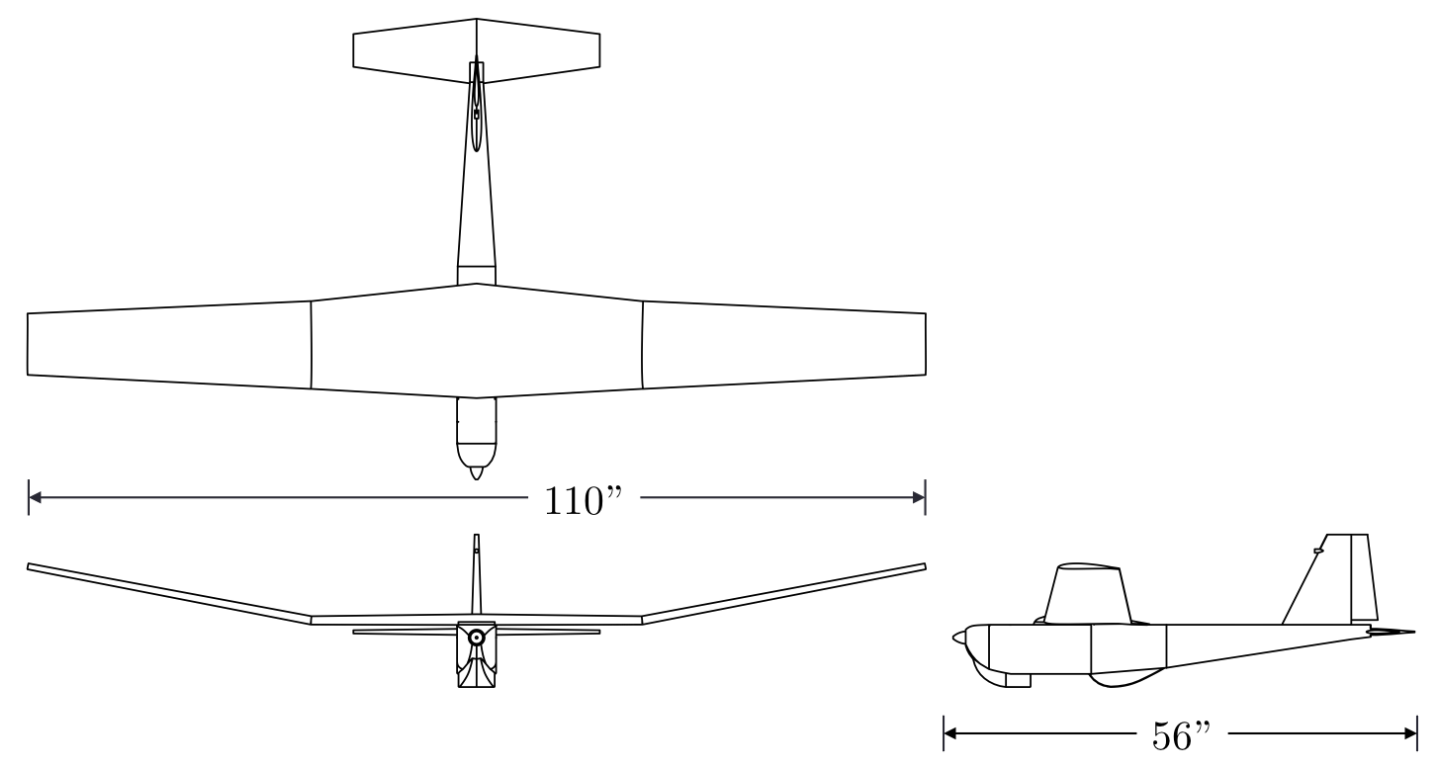

Figure 1.1: 3-view of AeroVironment Puma AE.

AeroVironment Inc.'s generous donation of an RQ-20A Puma AE system to the Cal Poly Aerospace Engineering Department's Autonomous Flight Laboratory allowed the Puma to be used as direct source geometry for this research. The Autonomous Flight Lab also obtained an FAA Certificate of Authorization (COA) allowing operation of the vehicle as a research platform in the national airspace. 


\subsection{Solar-Photovoltaic Energy Collection}

The behavior of photovoltaic solar cells with solar incidence angle is the driving principle behind this thesis. A photovoltaic cell only collects energy from radiation normal to its surfaces, and thus power output will vary as a cosine function.

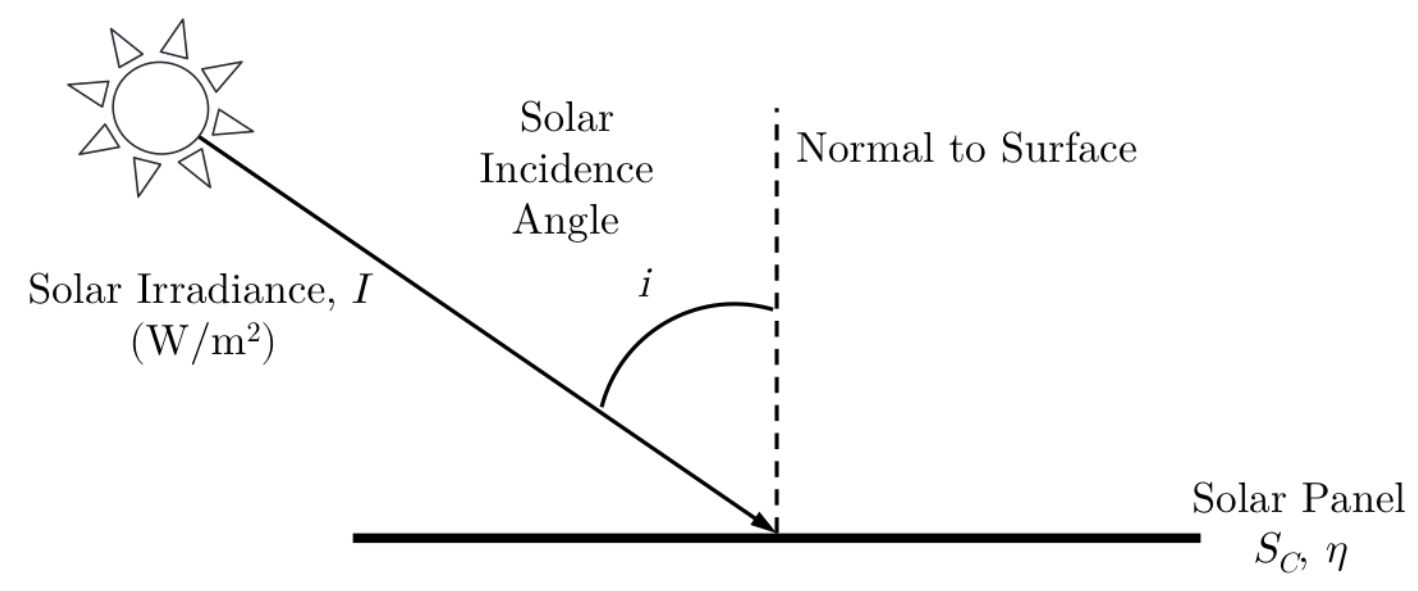

Figure 1.2: Characteristics of a photovoltaic solar cell in 2-D.

Since maximum energy collection is obtained at an incidence angle of zero, there is an incentive to use aircraft orientation to manipulate the incidence angle towards lesser values. On typical solar arrays, orientation is fixed and so collection efficiency is heavily dependent on sun position, which varies with time, day of year, geographic location, air temperature, and relative humidity. While these factors are still of critical importance to an accurate model of the general situation and will affect model results, aircraft show a unique ability to manipulate orientation of a solar array. 


\section{Chapter 2}

\section{AERODYNAMICS MODEL}

In this study, the approach taken to modeling asymmetric flight is to start from a known flight condition and aircraft geometry and work from there. This means that the aircraft's wind axis (heading, pitch, and bank angles) is set as an input. From there, the aircraft's body axis (angle of attack and angle of sideslip) is allowed to vary for trim purposes. As such, this is a point performance model that outputs total aircraft drag as well as the required rudder and stabilator deflections for trim. As it is desired to model different orbit shapes in this study, the aircraft must be allowed to fly asymmetrically both in a turn or at constant heading.

\subsection{Balance of Forces}

In order to maintain a selected heading in steady level flight despite an input bank angle, sideslip angle must be selected to produce a side force necessary to counteract the lateral lift component that arises when rolling the aircraft towards the sun. If the aircraft is allowed to turn in addition to flying asymmetrically, a centripetal force arises and is purely a function of the selected turn radius. The modeling approach taken here is to first determine the side force and lift vectors required for level flight, and then select the sideslip angle required to produce that side force and balance the aircraft. Simultaneously, angle of attack must be selected in order to produce the required lift force determined from the same force balance.

Determination of required lift and side force begins with an examination of a freebody-diagram of the forces at play on an aircraft flying asymmetrically at a given bank 
angle $\phi$, shown in Fig. 2.1.

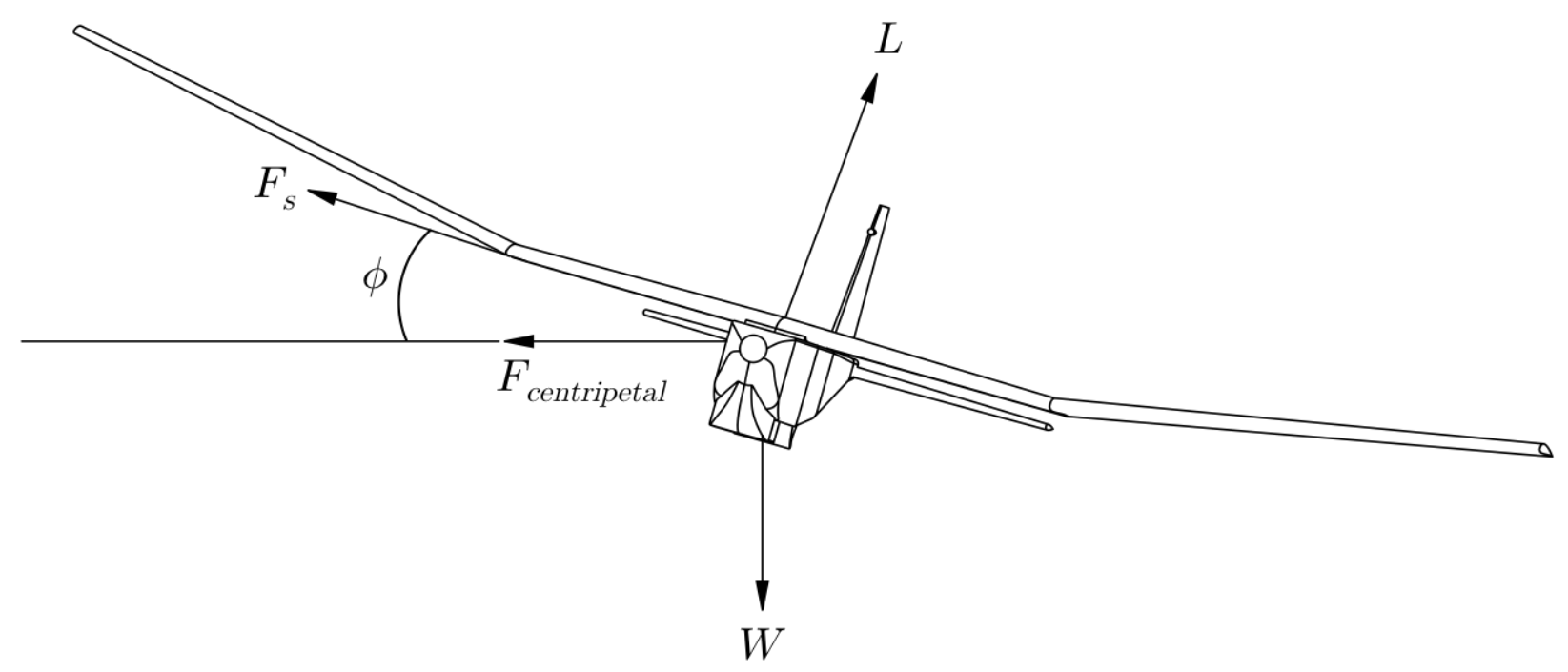

Figure 2.1: Forces acting on an aircraft in asymmetric flight.

Since centripetal force is treated as an input based on selected turn radius, the two unknowns in this problem are Lift $L$ and side force $F_{s}$. Using basic summation of forces, two equations can be generated:

$$
\begin{gathered}
F_{s}=L \tan (\phi) \\
L=\frac{W-F_{s} \sin (\phi)}{\cos (\phi)}
\end{gathered}
$$

Centripetal force can be calculated, for a steady level turn, from an input turn radius $R$ as follows:

$$
F_{\text {centripetal }}=\frac{m V_{\infty}^{2}}{R}
$$


Where $m$ is the mass of the aircraft and $V_{\infty}$ is the freestream velocity. For constantheading flight, turn radius is infinite and there is no centripetal force. Since the approach taken here requires an input flight condition and aircraft geometry, lift and side

force can both be customarily non-dimensionalized by dynamic pressure $q$ and planform reference area $S$ :

$$
\begin{gathered}
C_{L}=\frac{L}{q S} \\
C_{F_{s}}=\frac{F_{s}}{q S}
\end{gathered}
$$

With both lift and side force determined in terms of input bank angle, modeling of those forces can proceed in order to determine required angle of attack, sideslip angle, and corresponding drag increments.

\subsection{Modeling Lift and Longitudinal Stability}

Lift can be modeled purely as a function of angle of attack $\alpha$, but the production of lift from various sources on the aircraft must be considered in tandem with aircraft longitudinal stability. To maintain controlled asymmetric flight, moments in all three aircraft axes must be summed to zero by trimming the aircraft. This is accomplished in the longitudinal axis via elevator deflection. On the Puma, the horizontal stabilizer is an all-moving control surface and therefore considered a stabilator. Lift is produced by the main wing, fuselage, and horizontal stabilator as a function of angle of attack, and each source must be modeled. The rotated component of thrust due to angle of attack was treated as a contribution to total lift force, and modeled as an outside variable that was given an initial guess, and then iterated on for thrust equals drag. A basic representation of the Puma's geometry in the longitudinal axis can be seen in Fig. 2.2. 


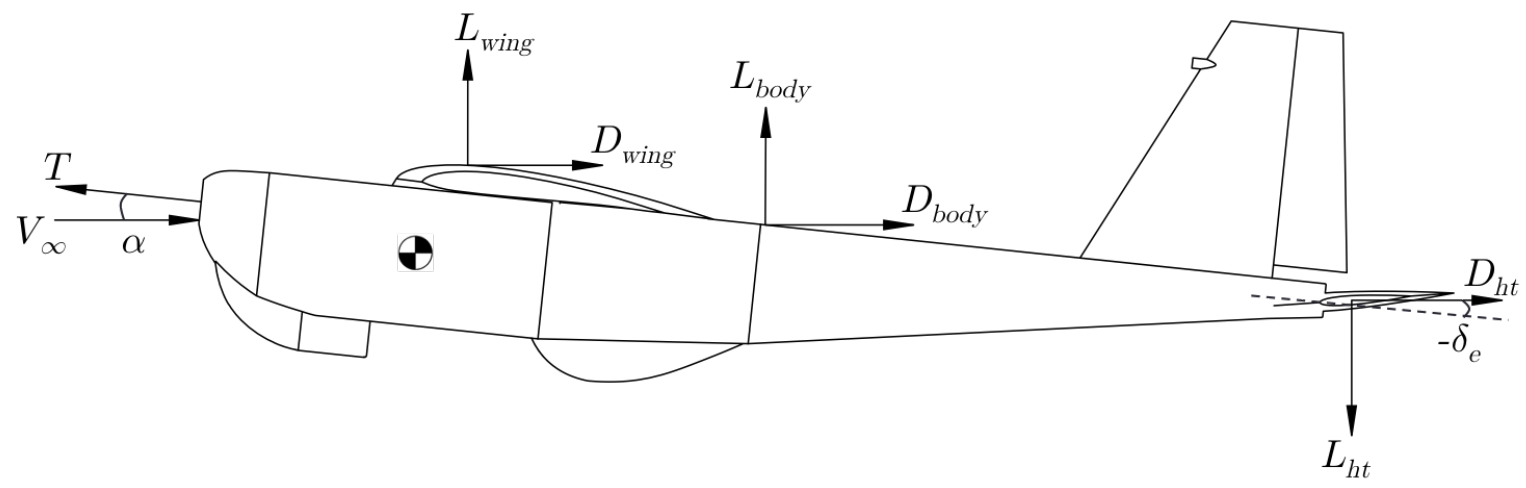

Figure 2.2: Forces considered for longitudinal stability.

\subsubsection{Vortex Lattice for the Main Wing and Stabilator}

To determine lift force, drag, and residual pitching moment on the main wing and stabilator, Tornado Vortex Lattice [4] was used. Tornado is a 3-D vortex lattice program implemented in Matlab and can output forces, aerodynamic coefficients, and stability derivatives [5]. While Tornado has the capability to perform trim calculations on its own, in this work it was used only to produce datasets of force and moment coefficients for each aerodynamic surface considered separately. With knowledge of where those forces and moments were computed on the input geometries, and of where those geometries are located in the Puma's configuration, trim calculations could be performed manually later on.

Vortex lattice methods model aerodynamic surfaces as infinitely thin sheets, or lattices, of discrete vortices and allow computation of pressure forces. These methods therefore neglect viscous effects such as skin friction and flow separation which necessitates restriction of input angles to those below which flow separation could be reasonably expected. Despite this, vortex lattice methods offer relatively fast computations and are widely used in early design phases or conceptual studies such as this one. 
With these limitations in mind, Tornado was used to build a database of values for lift, induced drag, and pitching moment for the Puma's main wing and stabilator. Input angle of attack was varied from -10 to 20 degrees for the main wing, and -20 to 20 degrees for the stabilator. While flow separation could be expected to occur somewhere above 10 degrees, this angle range was deliberately selected and for level flight at typical bank angles, angle of attack typically stays around 5 degrees, well within the linear range. Since the stabilator is all-moving, it was not necessary to define a control surface, but instead simply build a database of forces with angle of attack and later take care to interrogate that database for the appropriate stabilator deflection. A sample lift curve for the Puma's main wing is shown in Fig. 2.3.

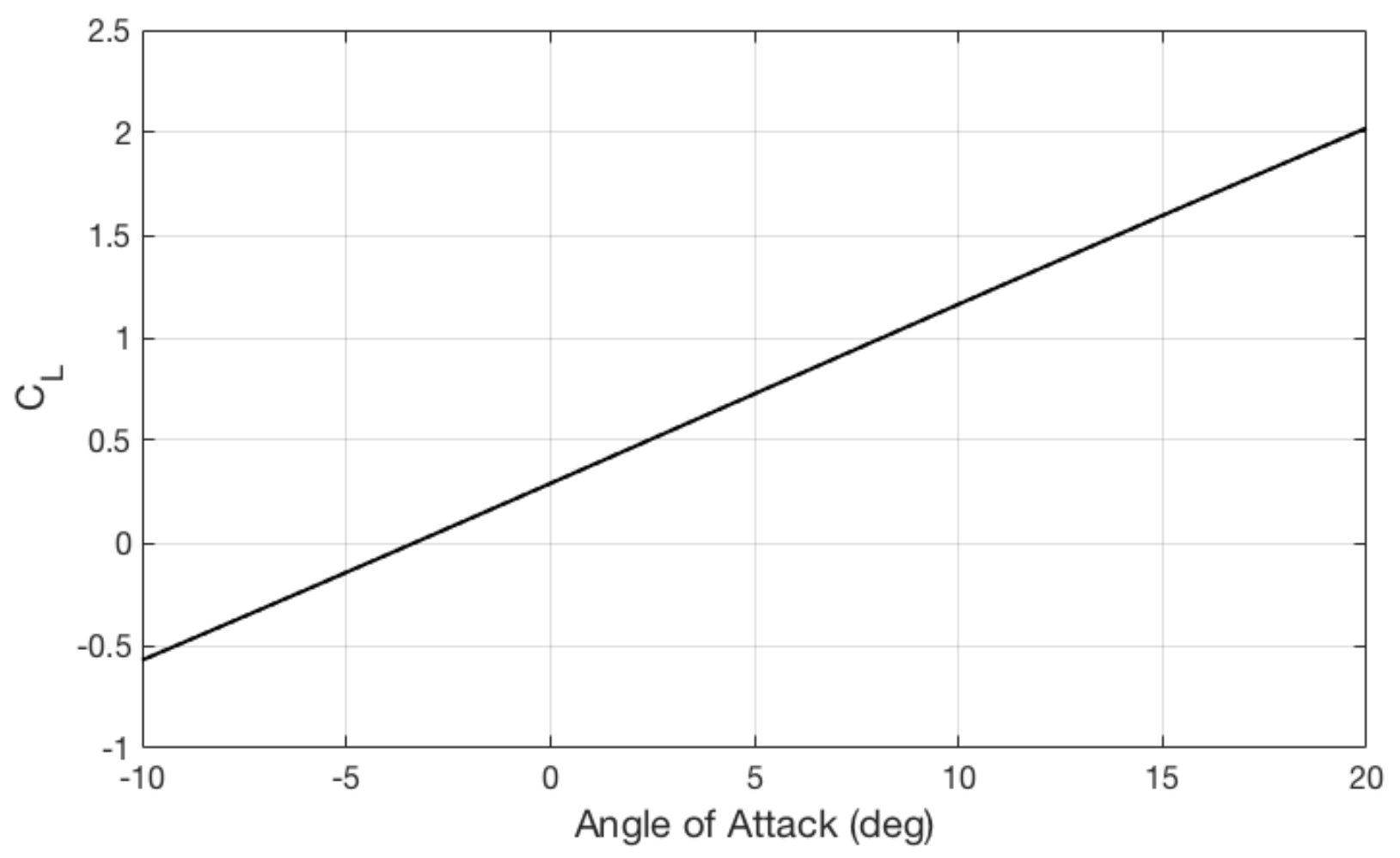

Figure 2.3: Lift curve for the Puma's main wing only. 


\subsubsection{The Cross-Flow Principle}

A critical component of this thesis is the determination of side force on a fuselage in sideslip. There are very few analytical methods that allow simple calculation of forces on an inclined fuselage-like body. However, one such method is the cross-flow principle as shown in [3]. This principle develops the relationship for the forces on a cylinder inclined with respect to oncoming flow and stipulates that at an angle of attack, pressure forces on cylindrical bodies depend only on the flow velocity component normal to their longitudinal axis. In this work, because of assumed symmetry, the cross-flow principle will be utilized for both calculation of lift on the fuselage due to angle of attack and side force due to sideslip angle. The cross-flow principle applied to the fuselage of the Puma AE in sideslip can be visualized in Fig. 2.4.

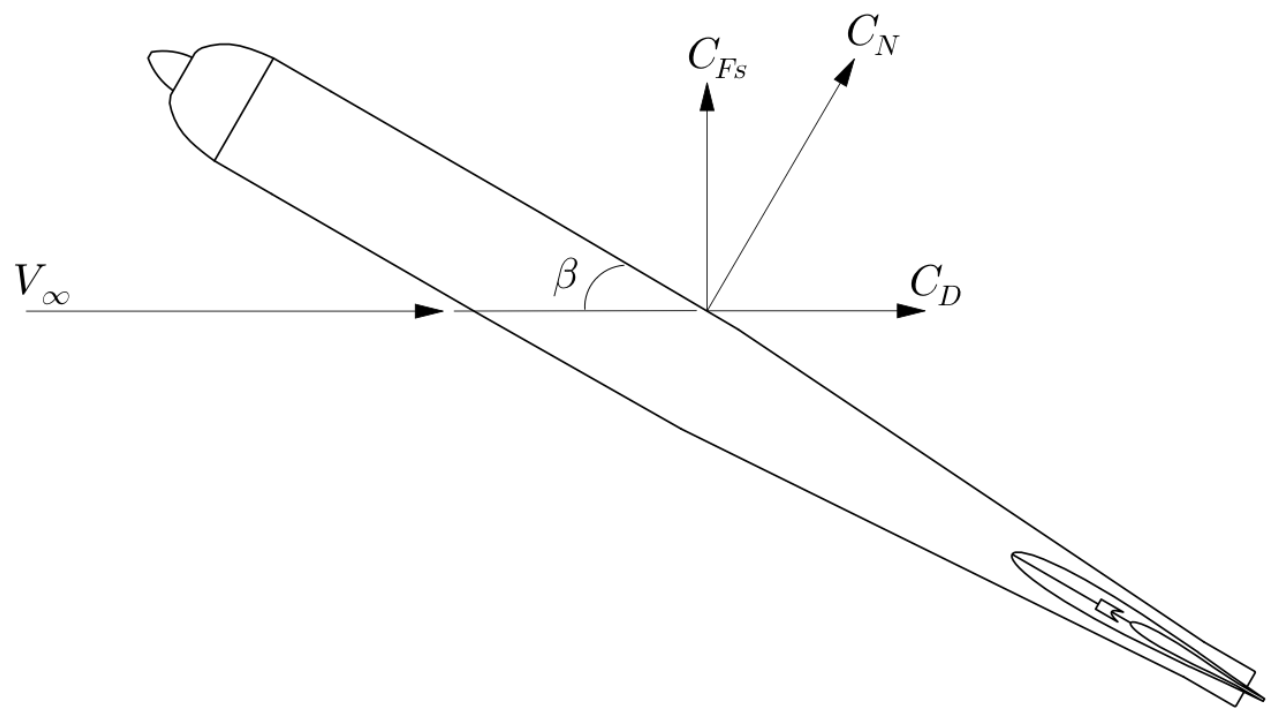

Figure 2.4: The cross-flow principle applied to the fuselage of the Puma AE.

The mathematical relationship developed for the normal force on the given inclined body in sideslip is as follows: 


$$
C_{N}=C_{D, \text { basic }} \sin ^{2}(\beta)
$$

Or, for calculation of lift on the inclined body due to angle of attack:

$$
C_{N}=C_{D, \text { basic }} \sin ^{2}(\alpha)
$$

$C_{D \text {,basic }}$ is based on the broadside area, not the frontal area, of the inclined shape upon which the cross-flow calculation is performed. In this case, the broadside area is a large rectangle. The cross-section of the Puma resembles a rectangular prism more than a cylinder, and so a $C_{D \text {,basic }}$ value of 2.05 was selected from [3].

The normal force can then be resolved into the side force (or, if using $\alpha$, lift) component using trigonometry:

$$
\begin{gathered}
C_{F_{s}, \text { fuse }}=C_{D, \text { basic }} \sin ^{2}(\beta) \cos (\beta) \\
F_{s, \text { fuse }}=C_{F_{s}, \text { fuse }} q S_{\text {broadside }}
\end{gathered}
$$

where $S_{\text {broadside }}$ represents an approximation of the Puma's broadside area as the length of its fuselage times its height.

\subsubsection{Calculation of Longitudinal Trim}

With all relevant forces in the longitudinal axis determined, moment about the aircraft's center of gravity can be calculated and trimmed to zero. For a given flight condition, center of gravity location, and angle of attack there is one stabilator deflection angle that will generate the necessary force to produce zero moment about the CG. 
For typical conventional configurations, the horizontal tail is down-loaded at positive angles of attack.

Longitudinal trim analysis begins in this approach by setting CG location in terms of static margin (SM), which is defined as follows in [6]:

$$
\mathrm{SM}=\frac{X_{\mathrm{np}}-X_{\mathrm{cg}}}{\bar{c}}
$$

where $X_{\mathrm{np}}$ and $X_{\mathrm{cg}}$ are the locations of the aircraft neutral point and center of gravity, respectively. $\bar{c}$ is the mean aerodynamic chord of the aircraft's wing. For a conventional, non-stability-augmented system, a positive static margin is typical, meaning that the aircraft is statically stable. In this thesis, a value of $10 \%$ was selected, and the CG was placed accordingly. Additionally, it was assumed that the aircraft neutral point and wing-only aerodynamic center were co-located; in reality an aft-tail configuration like the Puma would have an aircraft neutral point somewhat aft of its wing-body aerodynamic center.

With the CG location set, moments can be calculated about that CG as long as one knows the reference locations of each aircraft force with respect to the CG location. Typically, aerodynamicists use the aerodynamic center of a shape as its reference point about which moments and force are calculated, as the moment about that point is constant with angle of attack. However, in this case it is enough to know the force and moment about any reference point (as a function of angle of attack) as long as its location is known. In this analysis, it was assumed that all reference points were co-located vertically on the aircraft's body. A free-body-diagram of the longitudinal trim analysis is shown in Fig. 2.5.

Since lift and drag are functions of angle of attack, they must first be resolved to their component normal to the body axis, and then multiplied by the applicable 


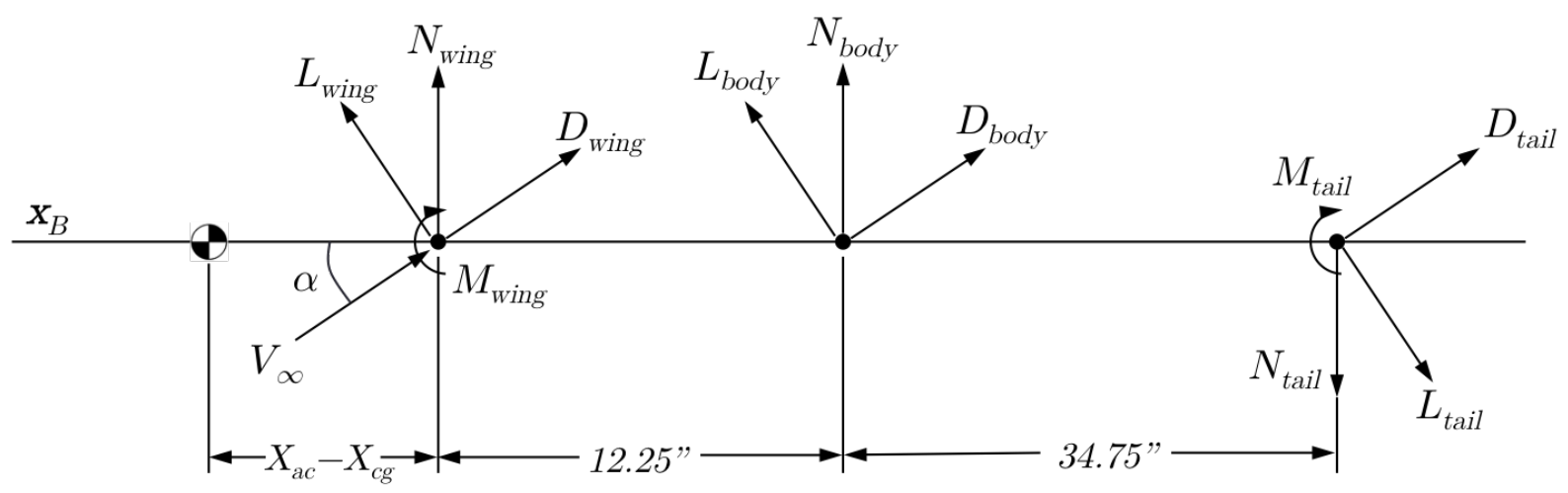

Figure 2.5: Free-body-diagram of longitudinal trim analysis.

distance from the center of gravity. The incidence angle of the horizontal stabilator is manipulated until it produces a force suitable for zero moment about the CG. Once this is accomplished for a sweep of angles of attack, a trimmed lift curve can be produced. Because it is a trimmed curve, each angle of attack and corresponding total aircraft lift coefficient has a stabilator deflection associated with it.

Since this method has no means of modeling non-linear effects such as flow separation and stall, the allowable angle of attack range in this study is limited as shown in Fig. 2.6. Generally, the model calls for angles of attack less than 5 degrees, which is well within the linear range of any typical aircraft's lift curve.

\subsection{Modeling Side Force and Directional Stability}

Side force and directional (yaw axis) trim must be considered as a function of sideslip angle. This process is somewhat analogous to the previous section, where lift and longitudinal stability was treated as a function of angle of attack. In this case, the relevant control surface is the rudder, which is used to generate the side force necessary off of the vertical tail to trim the moments in this axis to zero. A depiction of the forces considered in the yaw axis can be seen in Fig. 2.7 


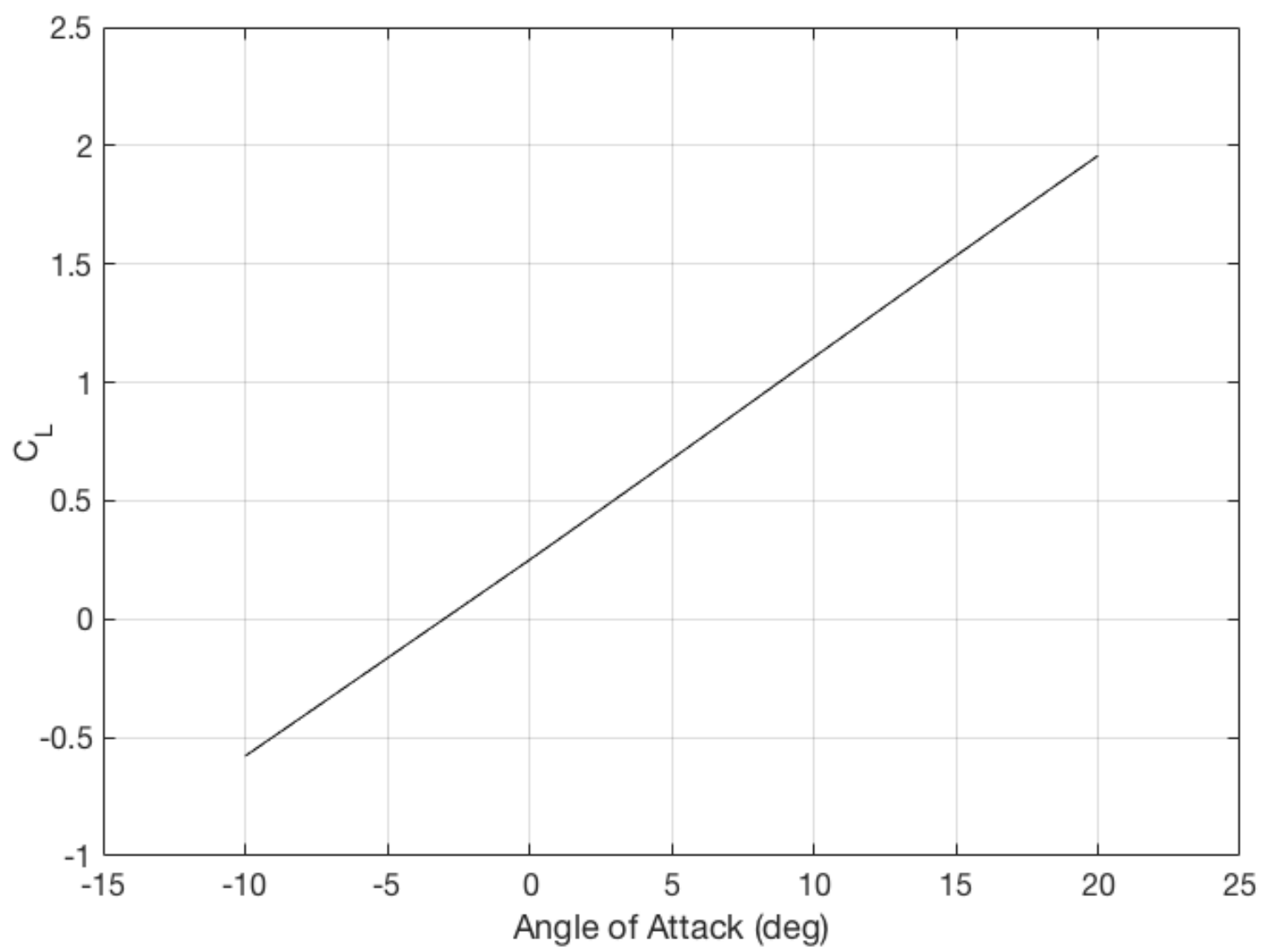

Figure 2.6: Trimmed lift curve.

\subsubsection{Calculation of Forces in the Yaw Axis}

Forces on the aircraft fuselage as a result of sideslip were again calculated using the cross-flow principle as described in Sec. 2.2.2. Care must be taken to resolve forces to the coordinate system relevant to the problem to be solved - forces resolved to the body axis must be used for trim calculations, while forces referenced to the wind axis should be used for force balance calculations.

For force and moment on the vertical tail, Tornado Vortex Lattice [4] was again used, as in Sec. 2.2.1. Tornado has the capability to define a control surface as a 


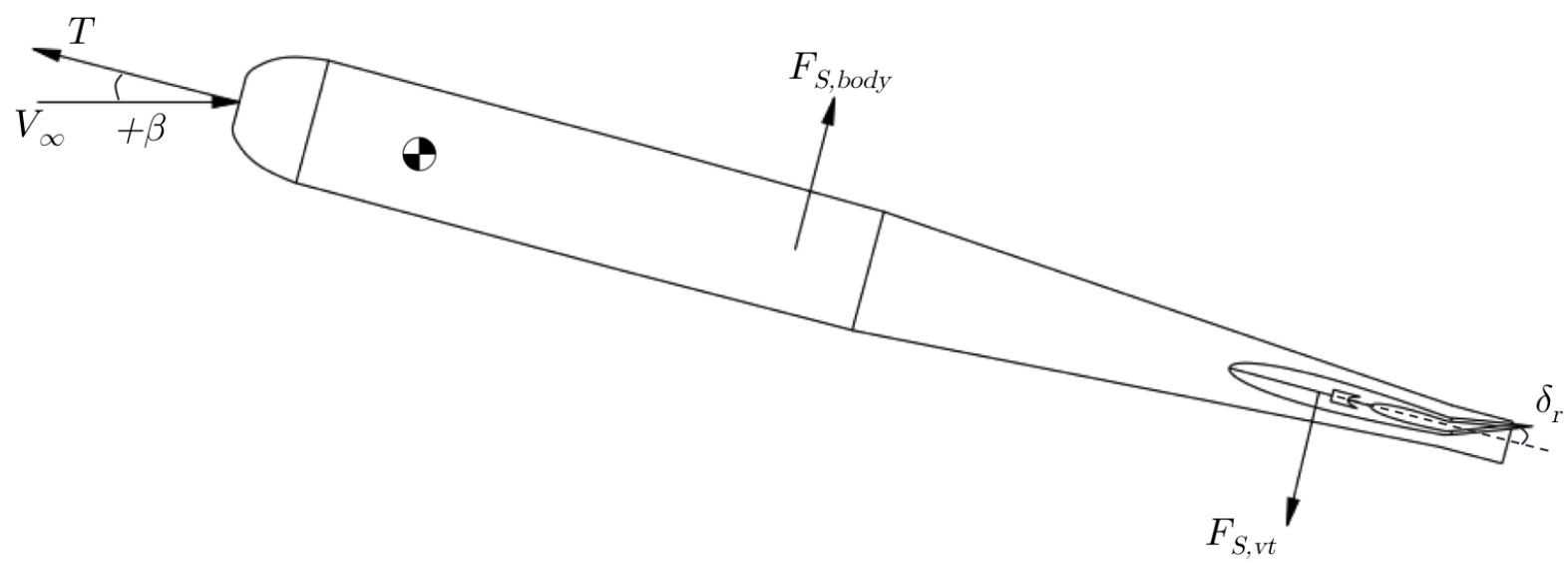

Figure 2.7: Free-body-diagram of directional trim analysis.

component of an existing aerodynamic surface. The vertical tail of the Puma consists of a NACA 0010 airfoil, and source measurements were used to define the rudder geometry in the model. A database of values for side force, yawing moment, and induced drag on the vertical tail was then built up for a sweep of both sideslip angle and rudder deflection. Rudder deflection angle was allowed to vary \pm 25 degrees, and sideslip angle varied \pm 15 degrees. Rudder deflection was computed in increments of 5 degrees, and sideslip angle in increments of 0.5 degrees. Linear interpolation was used to interrogate the model for values in between those increments.

Once again, the rotated component of the thrust vector due to sideslip angle was considered in the production of side force and treated as an outside variable of the entire aerodynamics model and iterated on for thrust equals drag.

\subsubsection{Trim in the Yaw Axis}

Similar to the calculations performed in Sec. 2.2.3, moments about the center of gravity in the yaw axis must also be summed to zero. In this case, for a given flight 
condition, CG location, and sideslip angle, there is one rudder deflection that will trim the aircraft. After trim analysis is performed, a trimmed side force vs. sideslip angle curve can be produced and is analogous to the trimmed lift curve from Sec. 2.2.3. It should be noted that in this work, positive rudder deflection is defined as trailing-edgeright as shown in Fig. 2.7. This rudder deflection produces a positive yawing moment in a nose-right wing-down coordinate system.

With the CG location already set from Sec. 2.2.3, the forces had to be located on the aircraft's body with appropriate dimensions. Once again, it was assumed that all reference points were co-located vertically on the aircraft's body. As in the longitudinal analysis, the center of pressure of the fuselage was assumed to be at the centroid of the rectangular prism it was modeled as. The reference point for the force and moment on the vertical tail was pulled from the vortex lattice code as the leading edge of the root chord of the vertical tail. A depiction of this analysis can be seen in Fig. 2.8.

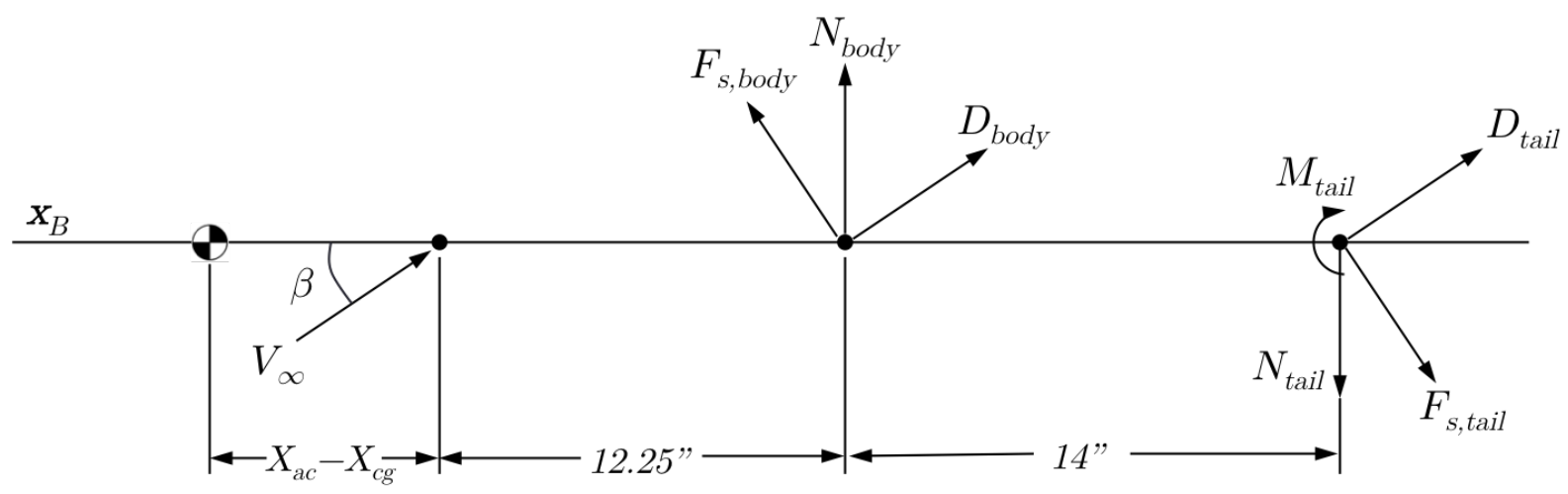

Figure 2.8: Free-body-diagram of directional trim analysis.

Side force and drag on each component must again be resolved to that component normal to the aircraft body axis in order to be computed as moment about the CG. Rudder deflection is manipulated in order to produce the normal force necessary to counter that of the body in sideslip. With this analysis completed for a sweep of sideslip angles, a trimmed side force coefficient vs. sideslip angle curve can be gen- 
erated as shown in Fig. 2.9. Since this is a trimmed curve, each sideslip angle and corresponding side force has one rudder deflection for trim.

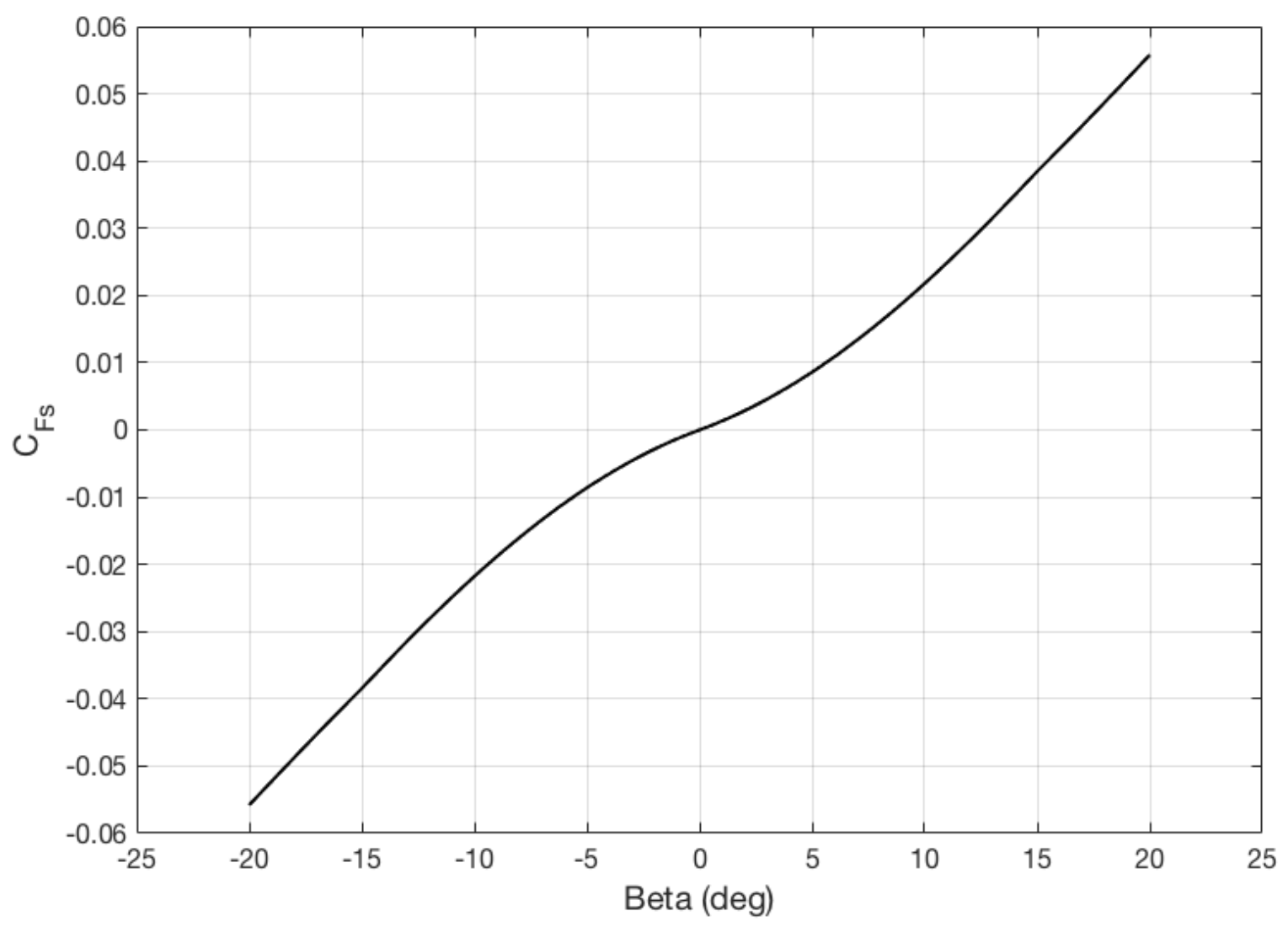

Figure 2.9: Trimmed side force curve.

The influence of the $\sin ^{2}$ function in the cross-flow principle can be seen in the center of the side force curve, as it is the only real source of side force apart from the influence of the vertical tail and rudder for trim. It is important to note that for this aircraft configuration and the 25 degrees of rudder deflection allowed, approximately 20 degrees of sideslip can be trimmed. Outside of that 20 degree range, the vertical tail and rudder do not have enough control power to trim the aircraft. This shows one of the key limits of the conventional configuration for an asymmetric flight profile.

For high sideslip angles, one should question the influence of viscous effects and eventual flow separation. While a limit of 20 degrees is placed here by trim constraints, 
Hoerner does show good agreement in [3] of the cross-flow principle with experimental data up to 90 degrees of body inclination angle.

\subsection{Correlating Bank Angle and Sideslip Angle}

At this point, it is important to develop an understanding of what range of input bank angles can be trimmed for steady level flight, since the goal of asymmetric flight is to use bank angle to point the aircraft toward the sun. The required forces developed in Sec. 2.1 depend only on bank angle, while the forces actually produced by the aircraft strongly depend on the selected flight condition, ultimately dynamic pressure. This suggests that flight condition will influence the efficacy of the asymmetric flight technique due to the fact that for a higher flight velocity or lower altitude, the range of bank angles that can be trimmed increases due to higher dynamic pressure.

For a typical Puma flight condition of 24 knots at $200 \mathrm{ft}$ MSL, it was found that the allowable range of 20 degree sideslip angle developed in this section corresponds with a bank angle range of approximately 5 degrees, meaning that this aircraft geometry can be "asymmetrically banked" at most 5 degrees towards the sun at the given flight condition. Later on in this study, the bank angle for best net power at a given flight condition and sun orientation will be determined.

\subsection{Parasite Drag}

With induced drag and drag due to sideslip determined in the previous sections, parasite drag on the aircraft must still be determined. Parasite drag is drag not due to lift, such as skin friction and form drag, and is primarily a function of flight condition and aircraft geometry. Classic drag buildup methods were used to develop a parasite drag model of the Puma AE, and a CAD model of the Puma, shown in Fig. 2.10, was 
Table 2.1: Calculated Parasite Drag Coefficient for the Puma AE.

\begin{tabular}{|l|c|}
\hline $\mathbf{C}_{\mathbf{D} 0}$ & Value \\
\hline Fuselage & 0.0155 \\
Wing & 0.0147 \\
Tail & 0.0035 \\
Total & 0.0337 \\
\hline
\end{tabular}

created in order to facilitate determination of wetted areas and other aircraft geometry parameters that this model relies on.

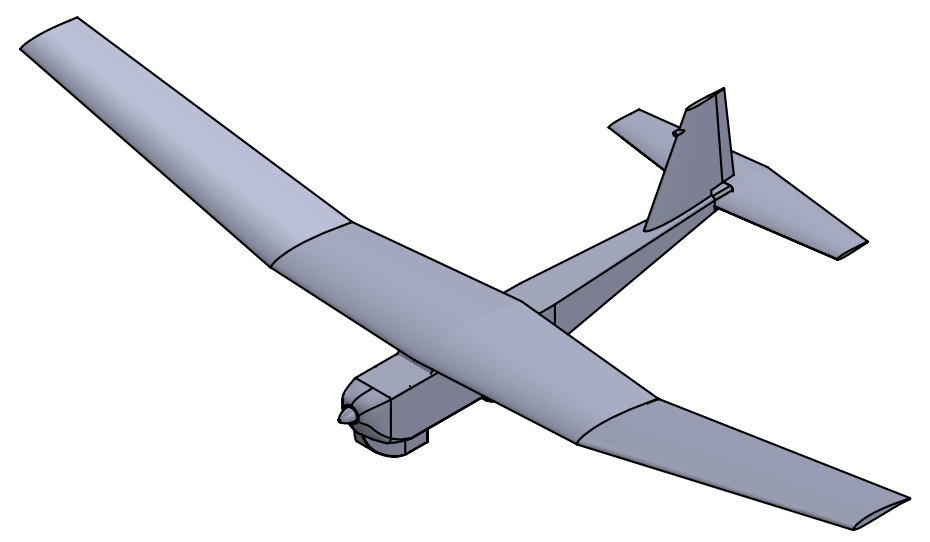

Figure 2.10: Simplified AeroVironment RQ-20 Puma AE geometry model.

The total aircraft parasite drag is determined by adding together approximations of drag for the body, wing, and tail, all weighted by the same reference planform area:

$$
C_{D 0, \text { total }}=C_{D 0, \text { body }}+C_{D 0, \text { wing }}+C_{D 0, \text { tail }}
$$

Individual calculation of parasite drag coefficients for the wing, body, and tail can be found in references such as [6]. Calculated values of $C_{D 0}$ for the Puma are shown in Table 2.1. 


\subsection{Asymmetric Flight Drag Polar}

With flight condition selected and required angle of attack, sideslip angle, and stabilator and rudder deflections determined from the previous sections, total aircraft drag can be calculated. All contributions to total drag have already been discussed, and now can be summed as shown:

$$
C_{D}=C_{D 0}+C_{D i}+C_{D, \text { sideslip }}
$$

Total induced drag, as shown previously, includes contributions from the fuselage, main wing, and horizontal stabilator. Total drag due to sideslip includes contributions from the fuselage and vertical tail with rudder deflection.

Conventionally, aircraft drag polars are built for zero sideslip angle. However, in this case it was key to develop a drag polar with sensitivity to sideslip as shown in Fig. 2.11 .

The non-linearity in drag increase with sideslip angle should be noted, as the 0, 10, and 15 degree cases were plotted. It is also shown that a fairly heavy penalty is paid in terms of drag for flying in sideslip, which will strongly penalize the asymmetric flight technique.

\subsection{Aerodynamics Model Validation}

A computational fluid dynamics (CFD) simulation was built in order to produce aerodynamic data for comparison with the analytical model presented here. There are a number of CFD software packages available, and STAR-CCM+ by CD-adapco [13] was used in this study. STAR-CCM+ is a cell-centered comprehensive CFD package 


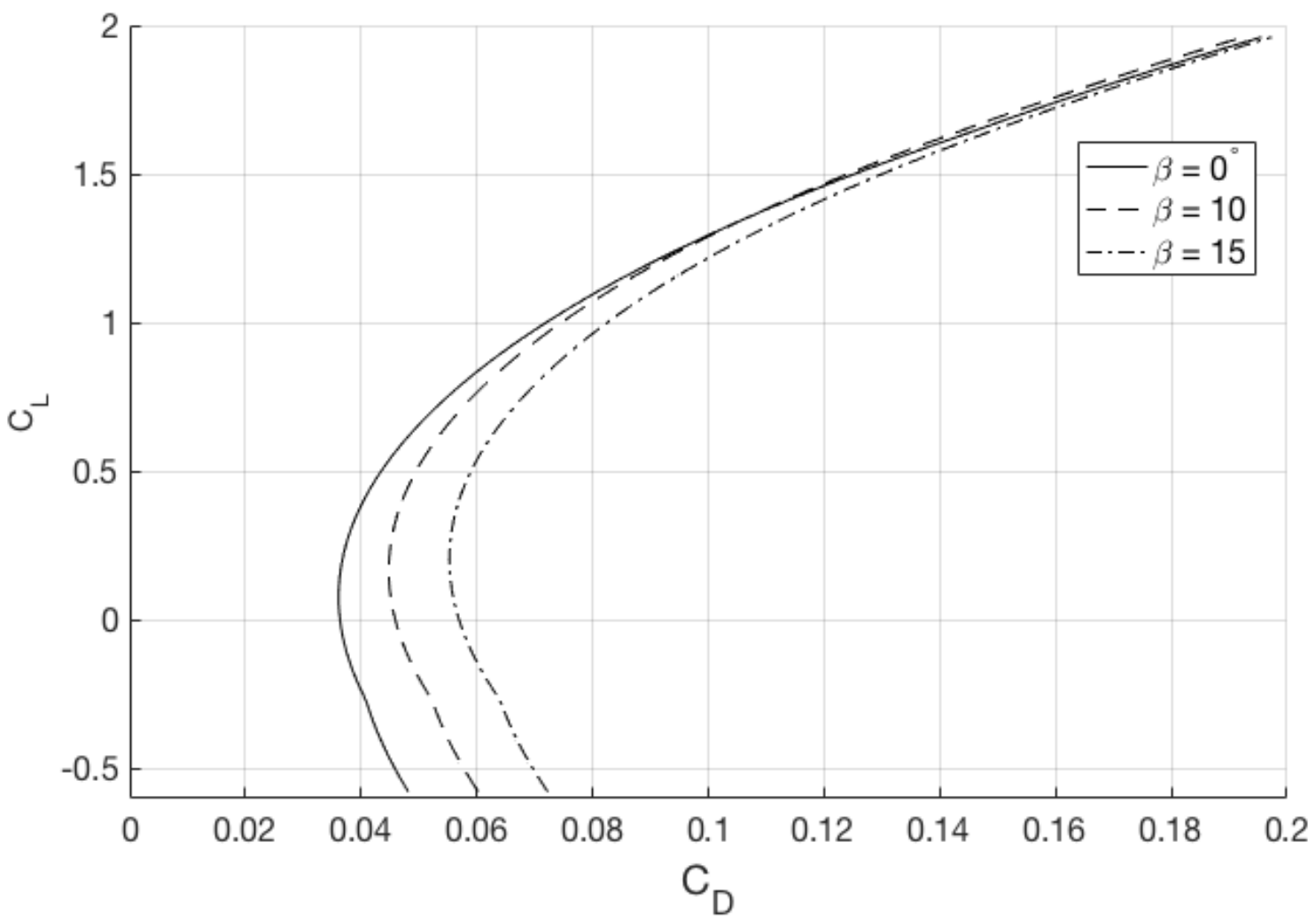

Figure 2.11: Asymmetric flight drag polar.

with both structured and unstructured mesh options. It is not the intention of this study to build full validation and agreement between the CFD simulation and analytical model, but instead to use CFD to provide a quantitative and qualitative insight into the accuracy of the aerodynamics model.

In this simulation, the Reynolds-Averaged Navier Stokes (RANS) solver was used with a hybrid unstructured mesh. The typical flight condition of the Puma is well within the incompressible flow regime, so constant-density flow was used along with an assumption of fully-developed turbulent flow throughout the entire flow field.

Initially, the goal of this study was to generate a simulation of the full aircraft such that for an input angle of attack and sideslip angle, model-calculated rudder and 
stabilator deflections could be applied in the CFD simulation to check for a trimmed aircraft. After a long process of trial-and-error, it was determined that limitations in cell count due to computational resources available precluded a full aircraft simulation. Instead, a symmetry plane through the longitudinal axis of the aircraft was used in order to improve resolution and achieve convergence, meaning only half of the aircraft was simulated in CFD; because of this symmetry assumption, analysis of the directional axis is impossible. However, angle of attack and elevator deflection can still be input to the CFD simulation for comparison with analytical model predictions.

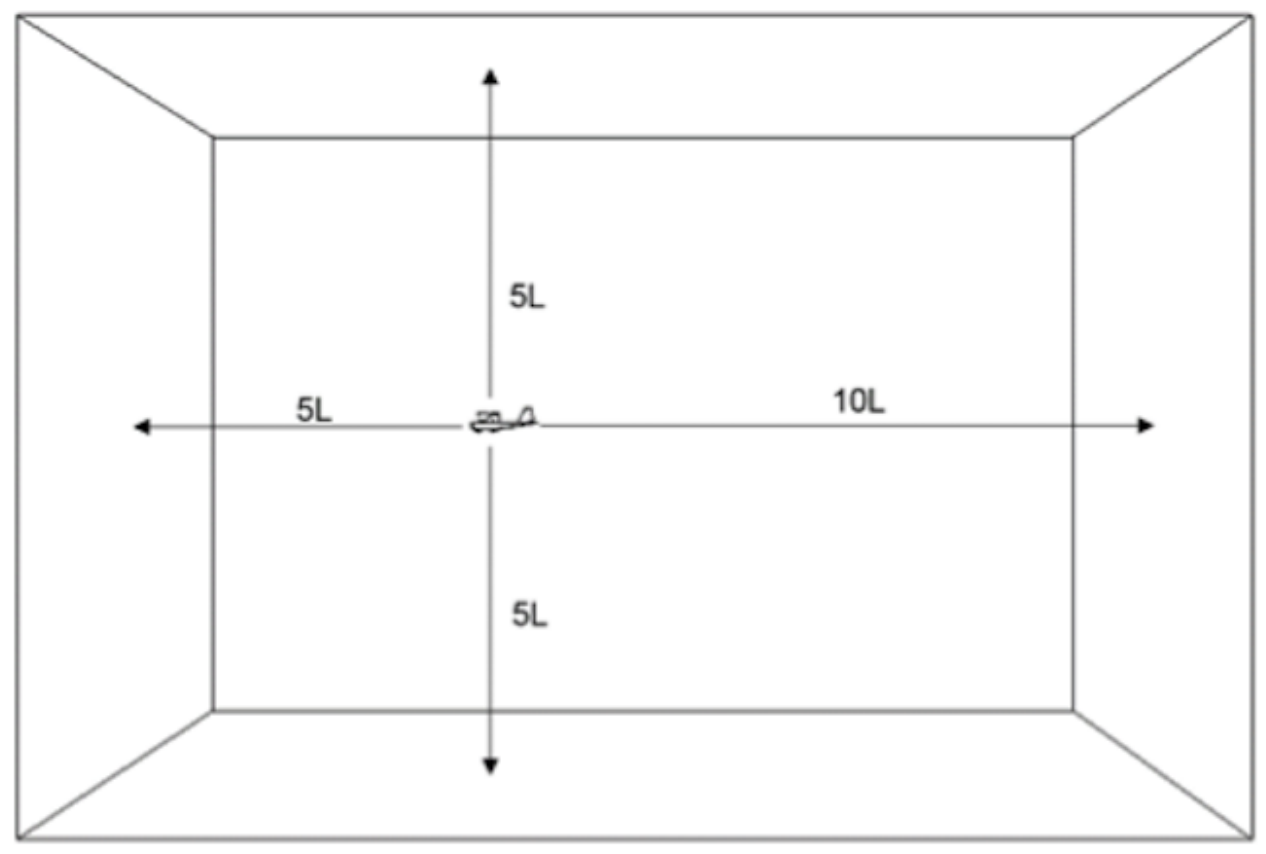

Figure 2.12: Locations of fluid boundaries in CFD simulation.

Fluid boundaries were set up as shown in Fig. 2.12, where $L$ indicates one vehicle length. The inlet was set as a velocity inlet of 24 knots, with a pressure outlet at freestream static pressure. Since it was desired to model the aircraft in a freestream condition, each boundary wall was placed far away from the aircraft and set as a slip wall. A symmetry plane was placed down the longitudinal axis of the aircraft. 
Table 2.2: Comparison of lift values for several CFD mesh resolutions.

\begin{tabular}{|l|c|c|}
\hline Mesh Cell Count & Lift (lbf) & $\Delta \mathbf{L}$ \\
\hline 15.7 million & 10.90 & $\mathrm{n} / \mathrm{a}$ \\
18.9 million & 10.87 & $-0.28 \%$ \\
23.5 million & 10.85 & $-0.18 \%$ \\
\hline
\end{tabular}

The trimmed mesh unique to STAR-CCM+ was used for this simulation, as it provides fast mesh times as well as cells that are nicely aligned away from the body. A volume control was placed around the aircraft, with surface and curvature controls on the aircraft surface itself. Once a satisfactory set of mesh settings had been determined, mesh convergence was examined by comparing lift values over three different mesh resolutions. Each mesh was considered converged when the lift value no longer varied within the hundredths digit, which corresponds with approximately a $\pm 0.05 \%$ difference between iterations. Typically, this level of convergence required around 3,000 iterations and took 3-4 days with the computational resources at hand for this study. One case was selected for inclusion in this thesis: an angle of attack of 5 degrees with the corresponding model-calculated stabilator deflection of -10.18 degrees (trailingedge up) for trim. The results of this mesh convergence study can be seen in Table 2.2 .

A few key flow features can be shown to illustrate the CFD solution. Negative pressure coefficients on the upper surface of the wing indicate lift creation in Fig. 2.13. Positive pressure coefficients on the upper surface of the stabilator show negative lift on the stabilator, as expected for trim of a conventional aircraft with positive angle of attack.

Comparison between values predicted by the aerodynamics model and values predicted by the CFD simulation can now be made. Of interest in this case are $C_{L}, C_{D}$, and pitching moment coefficient $C_{M}$. Lift and drag coefficients are shown in Table 


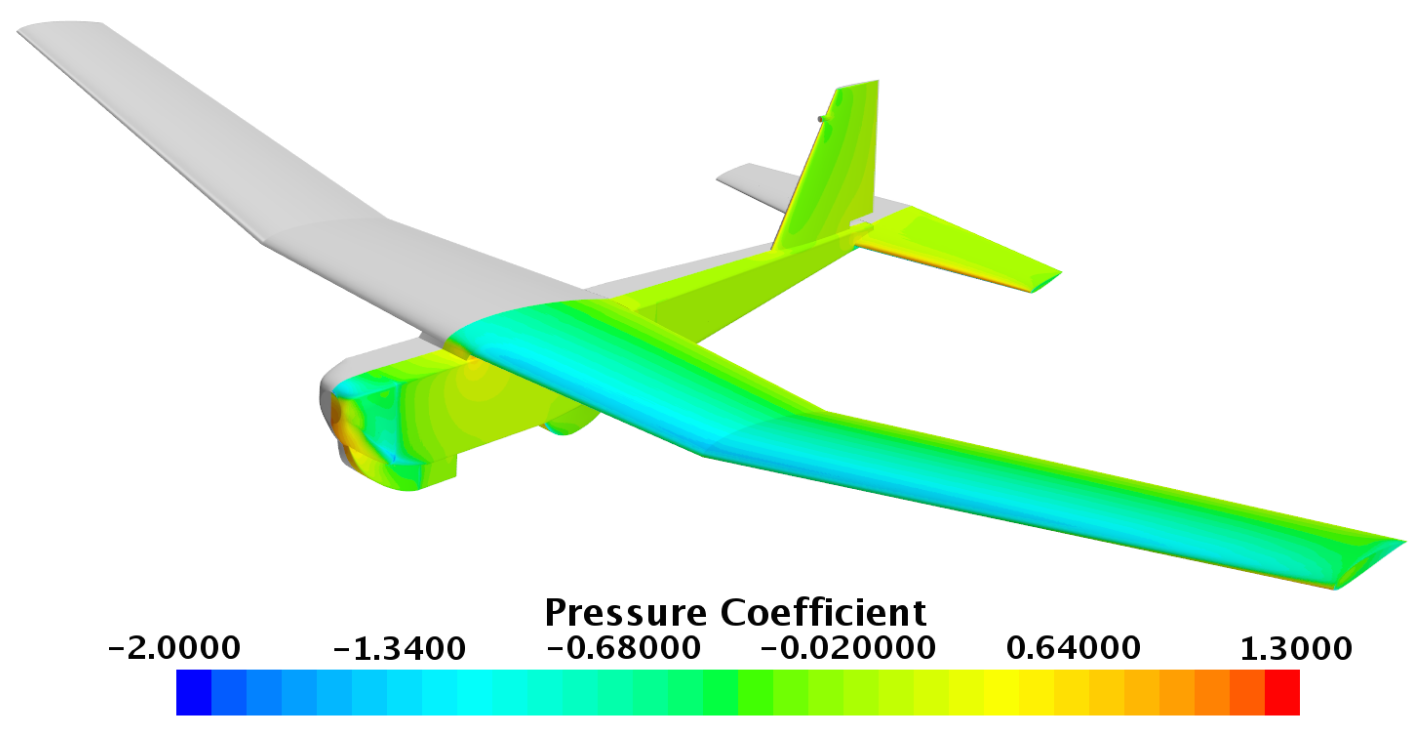

Figure 2.13: Contours of static pressure in Puma AE CFD simulation.

\section{3 .}

Table 2.3: Comparison of lift and drag coefficients between analytical and CFD models.

\begin{tabular}{|l|c|c|c|c|}
\hline Model & $\mathbf{C}_{\mathbf{L}}$ & $\Delta$ & $\mathbf{C}_{\mathbf{D}}$ & $\Delta$ \\
\hline Analytical & 0.671 & $\mathrm{n} / \mathrm{a}$ & 0.0509 & $\mathrm{n} / \mathrm{a}$ \\
CFD & 0.714 & $6.2 \%$ & 0.0652 & $24.6 \%$ \\
\hline
\end{tabular}

Calculation of pitching moment coefficient requires a reference location and is conventionally calculated about the aircraft CG. In the analytical model, it was assumed that the CG, and all other force reference locations, were vertically in-line with each other. Since the CFD simulation is fully 3-dimensional, this assumption breaks down. Instead, the pitching moment coefficient was calculated in the CFD model at a few different vertical (y-axis in this case) locations. Longitudinal location was still set at the same value as in the analytical model, and span-wise the CG is assumed to be directly on the centerline of the aircraft. By definition, the analytical model outputs a value of 0 pitching moment since stabilator deflection was calculated by the analytical model 
for zero pitching moment. Results for $C_{M}$ are shown in Table 2.4. Note that a y-value of 0 indicates a point at the top of the fuselage, $y$ increases negatively downwards, and that the Puma's fuselage is 6 inches tall at the assumed longitudinal location of the CG.

Table 2.4: Comparison of pitching moment coefficient between analytical and CFD models.

\begin{tabular}{|l|c|}
\hline Model & $\mathbf{C}_{\mathbf{M}}$ \\
\hline Analytical & 0 \\
CFD, y $=0$ & 0.035 \\
CFD, y $=-3 "$ & 0.042 \\
CFD, y $=-6 "$ & 0.049 \\
\hline
\end{tabular}

While the $C_{M}$ values extracted from CFD are non-zero, they are relatively close to zero and give at least some confidence in the outputs of the analytical model. Total lift and drag results are less encouraging, showing fairly high differences between the analytical and CFD models. However, the fact that numbers are even comparable between relatively high-fidelity CFD and the relatively low-fidelity techniques used in the analytical model, combined with the variety of simplifying assumptions made in that model, indicates that the modeling approach is sound.

\subsection{Calculation of Power Required}

With total aircraft drag determined, power required can be calculated simply by assuming steady level flight where thrust equals drag:

$$
P_{\text {req }}=T V=D V=C_{D} q S_{\text {ref }} V
$$

where $S_{\text {ref }}$ is the wing planform area and $V$ is flight velocity. While it is conventional to apply a propulsive efficiency factor to $P_{\text {req }}$, in this study the efficiency factors will be consolidated together and applied to power available in Sec. 3.1. With the 
calculation of power required, the aerodynamics model is complete, and attention can move to the calculation of power available. 


\section{Chapter 3}

\section{SOLAR-PHOTOVOLTAIC MODEL}

Solar energy collection is highly dependent on the incidence angle between the solar array normal and the vector of the sun's incident radiation. As the sun moves through the sky, both azimuth and elevation angle vary dependent on day of year, time of day, and latitude. Additionally, the roll, pitch, and yaw angles of an aircraft can be varied as desired, subject to trim and performance constraints. Therefore, in order to determine the energy collected by the aircraft, the energy output and motion of the sun along with solar cell performance must be modeled, and solar incidence angle calculated.

\subsection{Solar-Electric Propulsion Model}

In order to determine the power that is available to be used by the aircraft, the following equation is used:

$$
P_{\text {available }}=I \cos (i) S_{c} \eta_{\text {total }}
$$

where $I$ is the solar irradiance in $\mathrm{W} / \mathrm{m}^{2}, S_{c}$ is solar cell area, $i$ is the angle between the solar array normal and the line of sight to the sun, and $\eta_{\text {total }}$ is an efficiency value describing the total efficiency of the energy collection, storage, and propulsion systems. With a wing planform area of approximately $0.72 \mathrm{~m}^{2}, S_{C}$ for a notional solar Puma AE was selected to be $0.5 \mathrm{~m}^{2}$ which is $70 \%$ of available planform area. Using values from [7] shown in Table 3.1, $\eta_{\text {total }}$ is assumed to be $20 \%$. 


\section{Table 3.1: Assumed Puma AE energy conversion efficiencies}

\begin{tabular}{|l|c|}
\hline Component & Efficiency, \% \\
\hline Solar array & 35 \\
Maximum power point tracker & 97 \\
dc-dc converter & 95 \\
Battery charge & 95 \\
Battery discharge & 95 \\
Motor Controller & 95 \\
Motor & 85 \\
Propeller & 85 \\
$\eta_{\text {total }}$ & 20 \\
\hline
\end{tabular}

\subsection{Sun Angles}

Classical sun modeling equations shown in [8] can be used to describe the motion of the sun through the sky, culminating in the determination of the azimuth and elevation angles for any given combination of day of year, time of day, and location on Earth. First, calculate the year fraction, $\gamma$, in radians:

$$
\gamma=(n-1) \frac{360}{365} \cdot \frac{\pi}{180}
$$

where $n$ is the day of year, counting up from the beginning of the year. Declination angle $\delta$ is the angular position of the sun at solar noon, which can be calculated using an empirical approximation in radians:

$$
\begin{aligned}
\delta= & 0.006918-0.399912 \cos (\gamma)+0.070257 \sin (\gamma)-0.006758 \cos (2 \gamma) \\
& +0.000907 \sin (2 \gamma)-0.002697 \cos (3 \gamma)+0.00148 \sin (3 \gamma)
\end{aligned}
$$

Solar time (given here in hours) is used in all sun-angle relationships, and so standard time must be converted to solar time by first applying a constant correction of 4 minutes per degree for the difference in longitude (in degrees, negative to the west) 
between the observer's meridian and the prime meridian:

$$
t_{\mathrm{solar}}=t_{\mathrm{utc}}+4\left(\frac{\text { lon }}{60}\right)+\frac{\mathrm{EOT}}{60}
$$

where $t_{\mathrm{utc}}$ is the time in hours in coordinated universal time (UTC), and the Equation of Time (EOT), in minutes, is a second correction determined empirically and applied to account for the perturbations in Earth's rate of rotation:

$$
\begin{aligned}
E O T=229.2[0.000075+ & 0.001868 \cos (\gamma)-0.032077 \sin (\gamma) \\
& -0.014615 \cos (2 \gamma)-0.04089 \sin (2 \gamma)]
\end{aligned}
$$

When based on the prime meridian, the hour angle $\omega$ is the sun's angle in the sky (in radians), measured from the prime meridian. At solar noon, the hour angle is zero. Before solar noon, the hour angle is negative, and after solar noon, the hour angle is positive.

$$
\omega=\left(t_{\text {solar }}-12\right) \cdot 15 \frac{\mathrm{deg}}{\mathrm{hr}} \cdot \frac{\pi}{180}
$$

The zenith, elevation, and azimuth angles can then be calculated with the following:

$$
\begin{gathered}
z=\cos ^{-1}(\cos (\text { lat }) \cos (\delta) \cos (\omega)+\sin (\text { lat }) \sin (\delta)) \\
e=\sin ^{-1}(\cos (\text { lat }) \cos (\delta) \cos (\omega)+\sin (\text { lat }) \sin (\delta))=90^{\circ}-z \\
a=\frac{\omega}{|\omega|} \cdot \frac{\mid \cos ^{-1}(\cos (z) \sin (\text { lat })-\sin (\delta)) \mid}{\sin (z) \cos (\text { lat })}+\pi
\end{gathered}
$$


where zenith angle $z$ is calculated positive off vertical, elevation angle $e$ is calculated positive up from the horizon, and azimuth angle $a$ is calculated positive from North towards East.

\subsection{Solar Irradiance}

Solar irradiance describes the sun's raw power output that is incident on a unit area of surface. There are two main types of solar radiation: beam, sometimes called direct, and diffuse radiation. Beam radiation is that received from the sun without having been first scattered by the atmosphere, while diffuse radiation is received after its direction has been changed by atmospheric scattering. Total solar radiation can then be determined by summing both the beam and diffuse radiation.[8]

Calculation of the solar beam irradiance present on the Earth's surface must begin with calculation of the extraterrestrial beam irradiance, which varies with time of year due to the Earth-Sun distance. Given by Spencer, as cited in [8], extraterrestrial normal beam irradiance can be determined by:

$$
\begin{aligned}
I_{\mathrm{on}}=I_{s c}[1.000110+0.034221 \cos (\gamma)+0.00128 \sin (\gamma) & +0.000719 \cos (2 \gamma) \\
& +0.000077 \sin (2 \gamma)]
\end{aligned}
$$

where $I_{s c}$ is the solar constant, $1367 \mathrm{~W} / \mathrm{m}^{2}$. The normal beam irradiance on Earth's surface, $I_{\text {beam }}$ is then:

$$
I_{\text {beam }}=I_{\text {on }} \tau_{\text {beam }}
$$

where the beam atmospheric transmittance $\tau_{\text {beam }}$ is given by 


$$
\tau_{\text {beam }}=\frac{I_{\text {beam }}}{I_{\text {on }}}=r_{0} a_{0}^{*}+r_{1} a_{1}^{*} \exp \left(-r_{k} k^{*} m\right)
$$

Calculations on Earth's surface must account for losses due to the air mass, $m$, of the atmosphere, which is defined as the ratio of the mass of atmosphere through which beam radiation passes to the mass it would pass through if the sun were at the zenith directly overhead. As shown in [8], for zenith angles up to $70^{\circ}$, the following is a close approximation:

$$
m=\frac{1}{\cos (z)}
$$

However, for validity in zenith angles approaching $90^{\circ}$, losses due to curvature of the Earth can become significant. An empirical relation from Kasten and Young (1989), as cited in [8], can then be used:

$$
m=\frac{\exp (-0.0001184 h)}{\cos (z)+0.5057(96.080-z)^{-1.634}}
$$

where $h$ is aircraft altitude.

Returning to Eq. (3.12), the constants $r_{0}, r_{1}$, and $r_{k}$ are correction factors for different types of climates and seasons. As shown in [8], for midlatitude summer, $r_{0}=0.97, r_{1}=0.99$, and $r_{k}=1.02$. The constants $a_{0}^{*}, a_{1}^{*}$, and $k^{*}$ are derived by Hottel in [9] and are valid for altitudes less than $2.5 \mathrm{~km}$ and visibility greater than 23 $\mathrm{km}$ :

$$
\begin{aligned}
& a_{0}^{*}=0.4237-0.00821(6.0-h)^{2} \\
& a_{1}^{*}=0.5055+0.00595(6.5-h)^{2}
\end{aligned}
$$




$$
k^{*}=0.2711+0.01858(2.5-h)^{2}
$$

Diffuse irradiance can be calculated using

$$
I_{\text {diffuse }}=I_{\text {on }} \tau_{\text {diffuse }}
$$

where diffuse transmittance $\tau_{\text {diffuse }}$ can be determined from the empirical expression in [9]:

$$
\tau_{\text {diffuse }}=0.271-0.294 \tau_{\text {beam }}
$$

Finally, total solar irradiance can be calculated by adding the beam and diffuse components together:

$$
I=I_{\text {beam }}+I_{\text {diffuse }}
$$

\subsubsection{Alternate Model for Atmospheric Transmittance}

Another method for calculating the atmospheric transmittance exists, as developed in [12], and is sensitive to local temperature and humidity. Since these quantities are only available if specific weather reporting information exists for the selected location, the method presented earlier is still of value as location on Earth is the only required input. However, this alternate method was found of use in comparing model results with experimentally determined results, which are shown later in Sec. 3.5. The Apogee Instruments SP-422 solar pyranometer used to measure solar irradiance outputs results which agree to within 5\% of the "clear sky model" developed in [12]. In this thesis, this alternate model was used for comparisons with experimental data, and the previously 
developed model was used for all other point performance and mission calculations. In general, the method that relies only on location agrees to approximately $10 \%$ with experimental results, and those of the clear sky model.

The only difference in these two models is the method used to calculate atmospheric transmittance. The alternate model takes into account air temperature and relative humidity in determining the relative air mass that solar radiation must pass through before reaching the Earth's surface.

Required inputs for this model are relative humidity and air temperature and pressure measured at the location of interest. The equation for beam atmospheric transmittance is given as follows:

$$
\tau_{\text {beam }}=0.98 \exp \left[\frac{-0.00146 P}{K_{t} \sin (e)}-0.075\left(\frac{W}{\sin (e)}\right)^{0.4}\right]
$$

where $K_{t}$, the turbidity coefficient, is equal to 1.0 in clean air and $P$ is the atmospheric pressure in $\mathrm{kPa}$ at the selected point of interest. The precipitable water in the atmosphere, $W$, is calculated in $\mathrm{mm}$ :

$$
W=0.14 e_{a} P+2.1
$$

$e_{a}$ is the actual vapor pressure of the air in $\mathrm{kPa}$ and be calculated from input relative humidity $\mathrm{RH}$ and air saturation pressure $P_{\text {sat }}$ :

$$
e_{a}=\mathrm{RH} \cdot P_{\mathrm{sat}}
$$

With $\tau_{\text {beam }}$ determined from (3.21), diffuse transmittance is computed as follows: 


$$
\tau_{\text {diffuse }}= \begin{cases}0.35-0.36 \tau_{\text {beam }} & \text { for } \tau_{\text {beam }} \geq 0.15 \\ 0.18+0.82 \tau_{\text {beam }} & \text { for } \tau_{\text {beam }}<0.15\end{cases}
$$

These alternate definitions of beam and diffuse atmospheric transmittances can then be substituted as before into Eqs. (3.11) and (3.18) to obtain total solar irradiance.

\subsection{Incidence Angle}

Incidence angle of solar radiation on an aircraft's solar array is derived in this section. The method presented here was primarily developed by Edwards et. al. in [1]. For the purposes of this study, a flat solar array mounted to the top of the aircraft is assumed. The general approach is to use the dot product to find the cosine of the angle between a vector pointing from the Earth's surface to the sun and a vector normal to the notional solar array. This angle is the solar incidence angle.

The Earth surface coordinate frame is expressed in terms of North, East, and down:

$$
E \equiv\left[\begin{array}{c}
\text { North } \\
\text { East } \\
\text { Down }
\end{array}\right]
$$

The vector $\hat{s}^{E}$ is a unit vector in the Earth frame pointing towards the sun, expressed in terms of the solar azimuth and elevation angles. Azimuth angle is defined positive from North towards East, and elevation positive up from the horizon.

$\hat{s}^{E}$ can then be determined by a negative rotation of the 1-axis first by the elevation angle, then by the azimuth angle: 


$$
\hat{s}^{E}=R_{3}^{T}(a) R_{2}^{T}(e)\left[\begin{array}{l}
1 \\
0 \\
0
\end{array}\right]=\left[\begin{array}{c}
\cos (a) \cos (e) \\
\sin (a) \cos (e) \\
-\sin (e)
\end{array}\right]
$$

where the following rotation matrices are used and define a counterclockwise rotation about the selected axis, consistent with the right hand rule in [10]:

$$
\begin{aligned}
& R_{3}(a)=\left[\begin{array}{ccc}
\cos (a) & \sin (a) & 0 \\
-\sin (a) & \cos (a) & 0 \\
0 & 0 & 1
\end{array}\right] \\
& R_{2}(e)=\left[\begin{array}{ccc}
\cos (e) & 0 & -\sin (e) \\
0 & 1 & 0 \\
\sin (e) & 0 & \cos (e)
\end{array}\right]
\end{aligned}
$$

The aircraft body frame is now expressed out of the nose, out of the right wing, and down through the floor:

$$
B \equiv\left[\begin{array}{c}
\text { Nose } \\
\text { Right wing } \\
\text { Down }
\end{array}\right]
$$

The vector $\hat{n}^{E}$ is a unit vector in the Earth frame pointing opposite the aircraft's down direction, and is defined via a negative rotation of $\hat{n}^{B}$, as shown in Fig. 3.1, through the Euler angles roll $\phi$, pitch $\theta$, and yaw $\psi$ : 


$$
\hat{n}^{E}=R_{3}^{T}(\psi) R_{2}^{T}(\theta) R_{1}^{T}(\phi)\left[\begin{array}{c}
0 \\
0 \\
-1
\end{array}\right]=\left[\begin{array}{c}
-\sin (\psi) \sin (\phi)-\cos (\psi) \sin (\theta) \cos (\phi) \\
\cos (\psi) \sin (\phi)-\sin (\psi) \sin (\theta) \cos (\phi) \\
-\cos (\theta) \cos (\phi)
\end{array}\right]
$$

where the following rotation matrices are used, again defining a positive rotation consistent with the right hand rule as shown in [10]:

$$
\begin{aligned}
& R_{3}(\psi)=\left[\begin{array}{ccc}
\cos (\psi) & \sin (\psi) & 0 \\
-\sin (\psi) & \cos (\psi) & 0 \\
0 & 0 & 1
\end{array}\right] \\
& R_{2}(\theta)=\left[\begin{array}{ccc}
\cos (\theta) & 0 & -\sin (\theta) \\
0 & 1 & 0 \\
\sin (\theta) & 0 & \cos (\theta)
\end{array}\right] \\
& R_{1}(\phi)=\left[\begin{array}{ccc}
1 & 0 & 0 \\
0 & \cos (\phi) & \sin (\phi) \\
0 & -\sin (\phi) & \cos (\phi)
\end{array}\right]
\end{aligned}
$$

With definitions of both $\hat{s}^{E}$ and $\hat{n}^{E}$ in terms of appropriate input parameters, the solar incidence angle can be calculated using the dot product:

$$
\cos (i)=\hat{s}^{E} \cdot \hat{n}^{E}
$$

Which can be expanded using Eqs. (3.26) and (3.30) to

$$
\begin{aligned}
\cos (i)= & -\cos (a) \cos (e) \sin (\psi) \sin (\phi)-\cos (a) \cos (e) \cos (\psi) \sin (\theta) \cos (\phi) \\
& +\sin (a) \cos (e) \cos (\psi) \sin (\phi) \\
& -\sin (a) \cos (e) \sin (\psi) \sin (\theta) \cos (\phi)+\sin (e) \cos (\theta) \cos (\phi)
\end{aligned}
$$




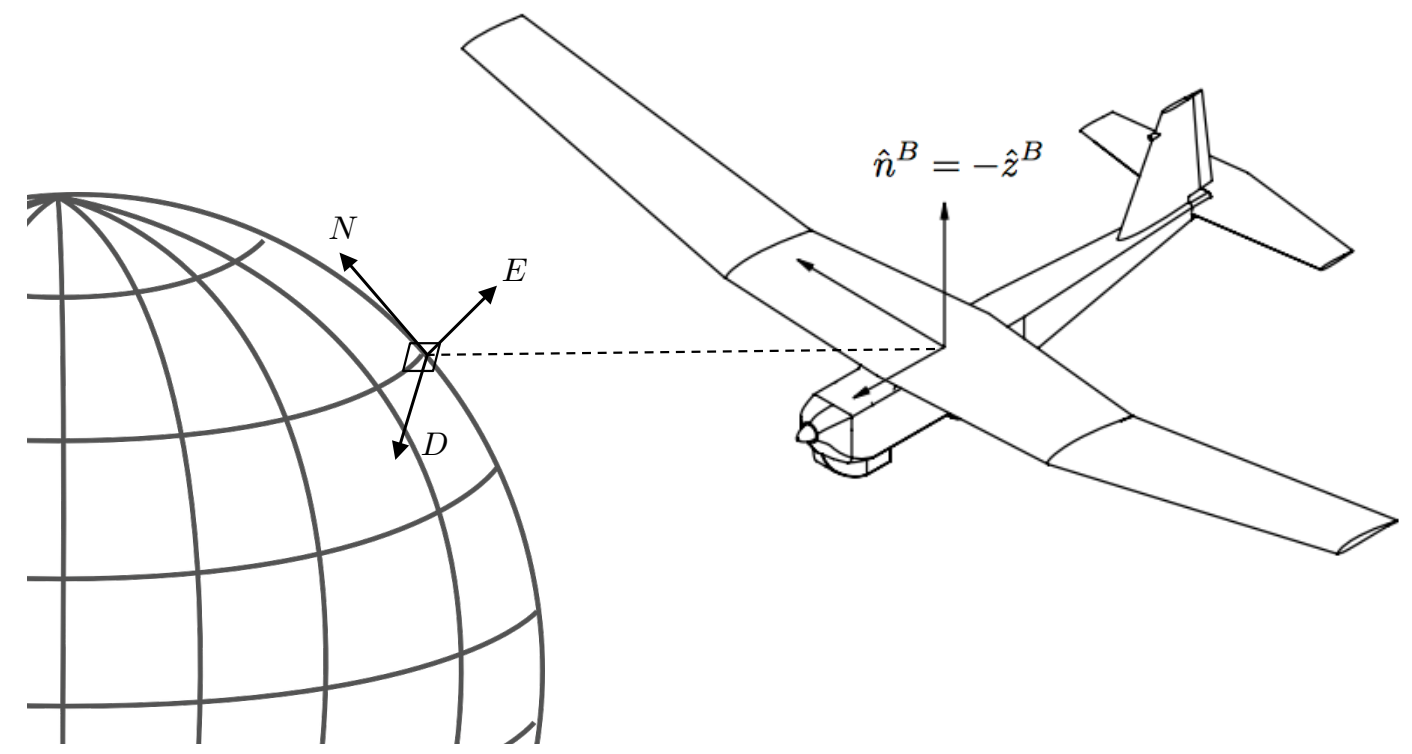

Figure 3.1: The Earth-fixed NED and aircraft body coordinate system.

There are two special cases where this value of $\cos (i)$ must be overridden. In sunset or nighttime conditions, elevation angle is negative. In this case, $\cos (i)$ is set to zero and no solar energy is collected. Similarly, if $\cos (i)$ is negative, the sun is shining on the bottom of the solar array, blocked by the aircraft structure, and no energy can be collected. While further shadowing analysis is neglected in this study, resources such as [11] exist which could be used to improve model fidelity.

With these modifications, $\cos (i)$ can be used for comparison between different combinations of aircraft orientation and sun position, independent of specific air vehicle (or solar array) characteristics. This term can therefore be thought of as a measure of solar collection efficiency relevant only to the maneuvers of the aircraft. It can also be used in Eq. (3.1) to obtain units of power. 


\subsection{Solar-Photovoltaic Model Validation}

In an effort to improve confidence in the function of the solar-photovoltaic model, a ground experiment was designed and executed in order to generate experimental solar irradiance data for correlation with model output values. A solar sensor called a pyranometer is an instrument for measuring total solar irradiance (beam and diffuse) on a planar surface, and works by producing a voltage from an internal detector that is a function of incident radiation [8].

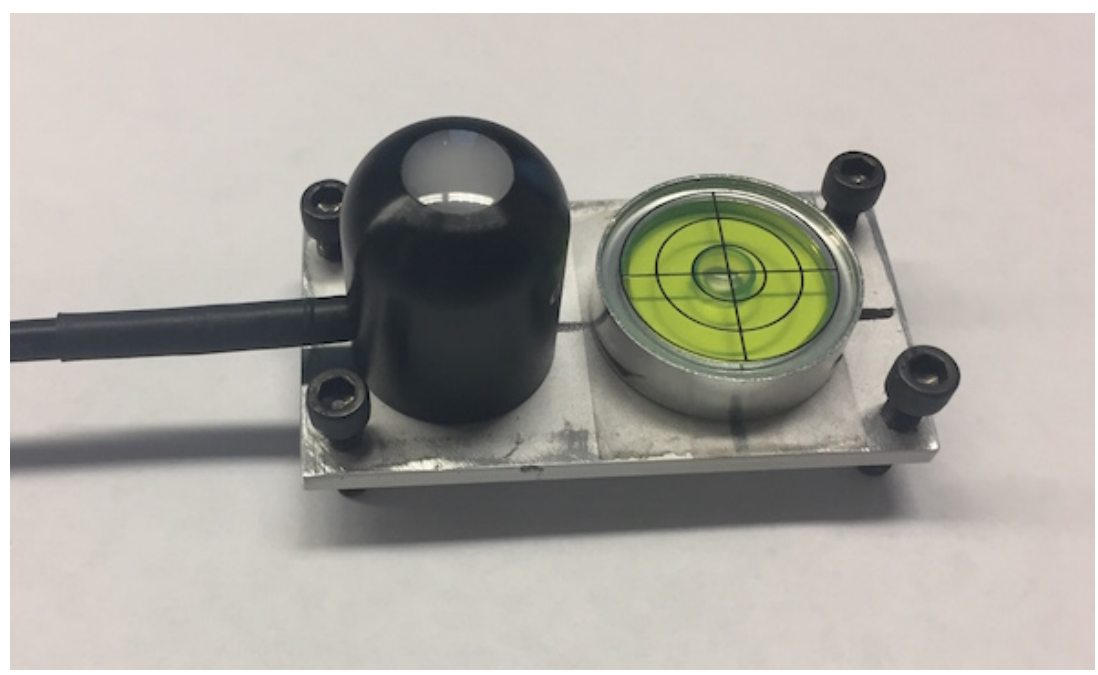

Figure 3.2: Experimental apparatus for the solar-photovoltaic model ground test.

In this study, the Apogee Instruments SP-422 smart pyranometer [16] was selected for its cost, accuracy, and integrated sensor/data logger solution. The sensor is calibrated to maintain a $\pm 5 \%$ accuracy from the Clear-Sky Calculator [17], a website developed and maintained by Apogee Instruments with similar outputs to the model developed in this study. When the alternate atmospheric transmittance model of Sec. 3.3.1 is used, irradiance results show very close agreement with the Clear-Sky Calculator and also the experimental results obtained with the SP-422.

Before conducting an experiment, effort must be taken to ensure that the sensor is 
placed level with the horizon, or in some known orientation. For this purpose, a simple leveling plate was constructed where the sensor was mounted along with a bubble level. Four screws placed in the corners of the plate provide adjustable-height feet used to level the plate on whatever surface it was placed on. The apparatus is shown in Fig. 3.2 , with the SP-422 on the left and the bubble level on the right.

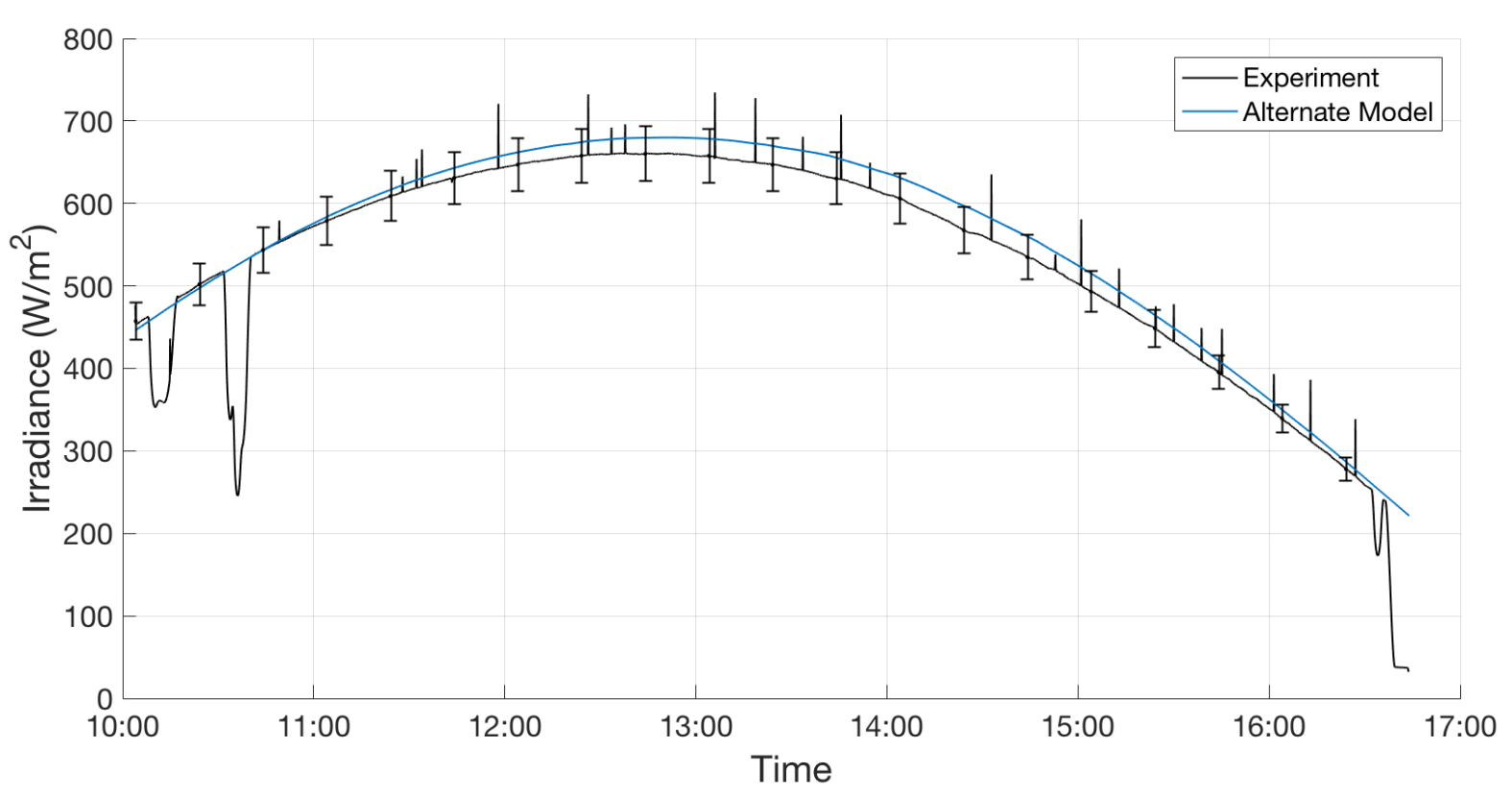

Figure 3.3: Irradiance vs. Time of Day for the solar sensor ground test performed 2 Nov. 2016, comparing experimental and alternate model data.

The sensor was placed outside on 2 November 2016 from approximately 10:00 to 17:00 in a location where shading did not occur until just before the experiment was ended. The drop in irradiance values for the experimental data may be attributed to momentary cloud cover; on the whole the day selected showed very clear skies. Figure 3.3 shows good agreement, within the manufacturer's stated error bounds as shown, between the experimental data and the alternate atmospheric transmittance model of Sec. 3.3.1.

The primary model, where atmospheric transmittance is based on season and geographic location alone, agrees with the experimental data to approximately $10 \%$, as 
shown in Fig. 3.4. While the alternate atmospheric transmittance model shows superior accuracy when compared to experimental data, it requires knowledge of both temperature and relative humidity at the location of interest, which are time-varying throughout the day. The primary model is more easily used to produce general results, which is in line with the scope and goals of this study, while still showing acceptable accuracy. The primary atmospheric transmittance model, in combination with the rest of the solar-photovoltaic model, is used for all of the results shown in this paper.

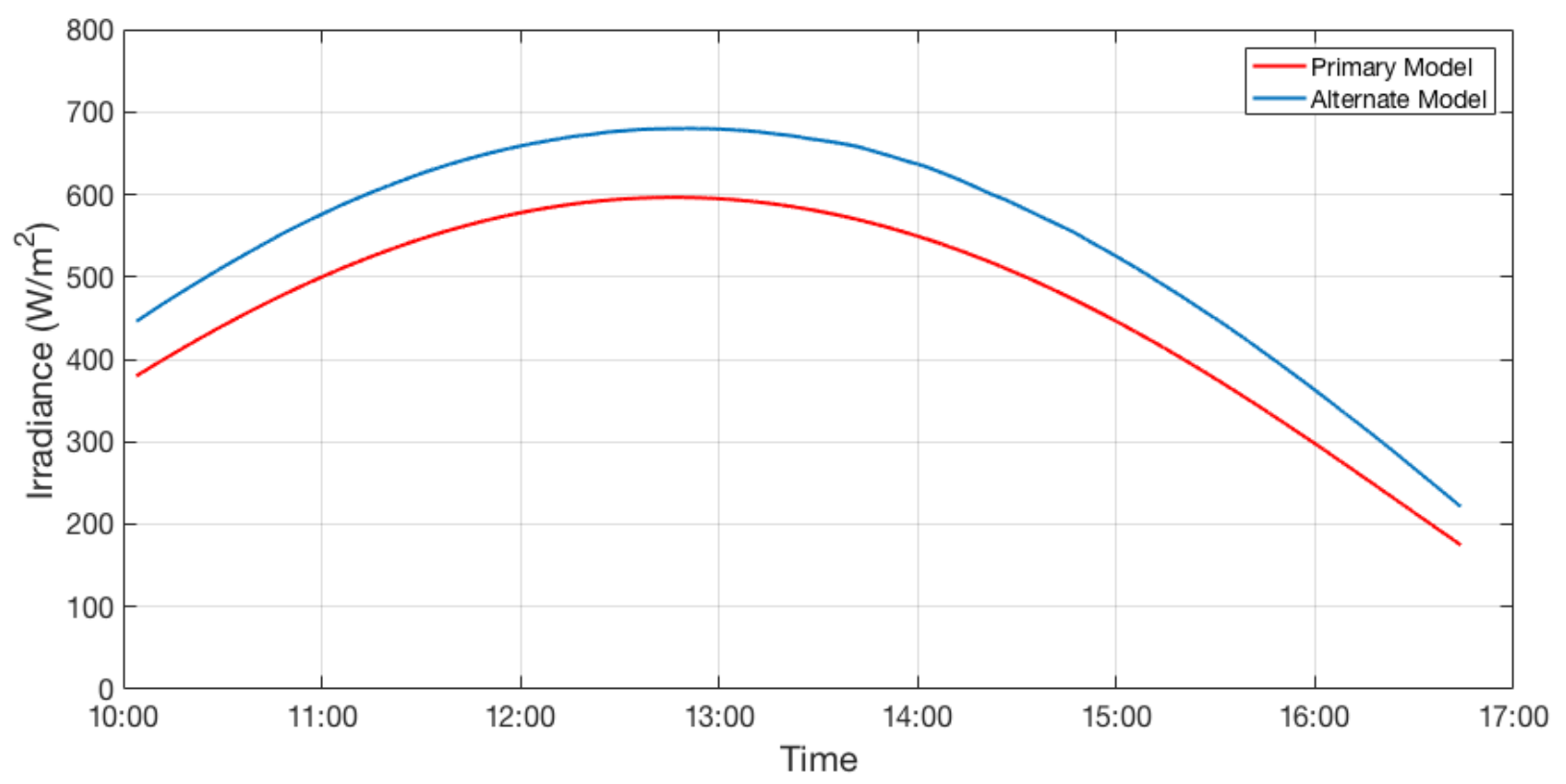

Figure 3.4: Irradiance vs. Time of Day, comparing primary and alternate model data.

Before plotting, modeled irradiance values must be corrected for incidence angle effects. In essence, power available (Eq. (3.1)) is computed for an efficiency factor and solar cell area of 1 . Therefore, the analytical data shown in this section relies on both the solar model and incidence angle models, and confidence can be built on the results of both. 


\section{Chapter 4}

\section{POINT PERFORMANCE RESULTS}

With both the aerodynamics model and the solar-photovoltaic model completed, initial results in a point performance context can be generated. Since the key trade-off of this study is between power available from the solar cells versus power required to fly the aircraft at a given condition and orientation, the parameter of interest in this section is primarily net power:

$$
P_{\text {net }}=P_{\text {available }}-P_{\text {required }}
$$

With the above definition of net power, it is worth noting that a positive value indicates that there is an excess of solar energy over that required to fly the aircraft, and any notional flight batteries would be charging during flight. With a negative value, the opposite is true, and batteries would be required to discharge in order to make up the remainder of the required energy for flight.

\subsection{Constant-Heading Results}

The model was then evaluated across the range of allowable bank angles in constantheading flight to find the bank angle for best net power on the summer solstice in San Luis Obispo, CA. Flight altitude and velocity were $200 \mathrm{ft}$ MSL and 24 knots, a typical flight condition for the Puma. It is important to note that in this first set of results, as shown in Fig. 4.1, the aircraft's heading was set at each time such that the sun was placed directly off of the right wing of the aircraft (i.e., the aircraft heading angle was set to be the solar azimuth angle minus 90 degrees), so that banking the solar array into 
the sun would have the most direct effect on power available. This forms the best case for asymmetric flight.
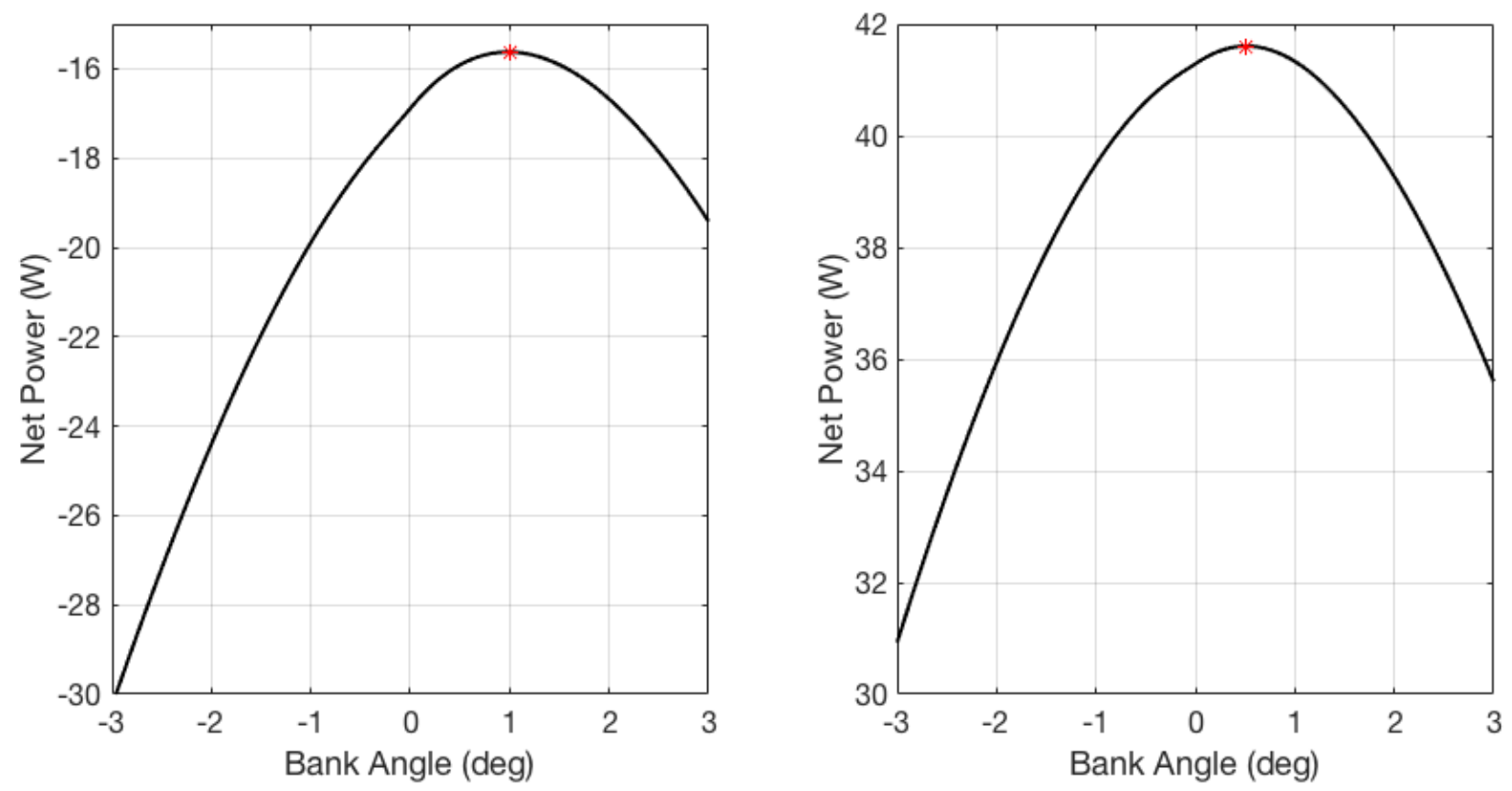

Figure 4.1: Net power for the Puma AE flying in San Luis Obispo, CA on the summer solstice at 8:00 AM (left) and 12:00 PM (right).

The calculation of net power with bank angle at two different times in Fig. 4.1 corresponds with common sense expectations. As the day approaches noon, elevation angle increases as the sun is higher in the sky. It is reasonable then that there is a smaller optimal bank angle for best net power at noon than early in the morning. Also, best net power increases in this time frame since better incidence angles will be found as solar elevation angle increases.

Even in the early morning, and with best case for solar radiation on the summer solstice, it is shown that the optimal bank angle for asymmetric flight is quite low, approximately 1 degree in the morning, decreasing to about 0.5 degrees at noon. This result is not unreasonable given the harsh penalty to asymmetric flight shown in the drag polar of Fig. 2.11. However, the asymmetric flight technique does show potential 
promise since there is a non-zero bank angle for best net power. It should be noted that the actual magnitudes of net power and optimal net bank angle are heavily dependent on aircraft specific parameters, although effort was made to base these results on an aircraft representative of the sUAS class.

In a continuing test of model validity, the same type of results were plotted and shown in Fig. 4.2 for noon and 4:00 PM. As the time of day increases past solar noon, elevation angle decreases and one expects net power to decrease, while similarly to the morning case, the bank angle for best net power should increase. The model therefore reacts as expected for times throughout the day.
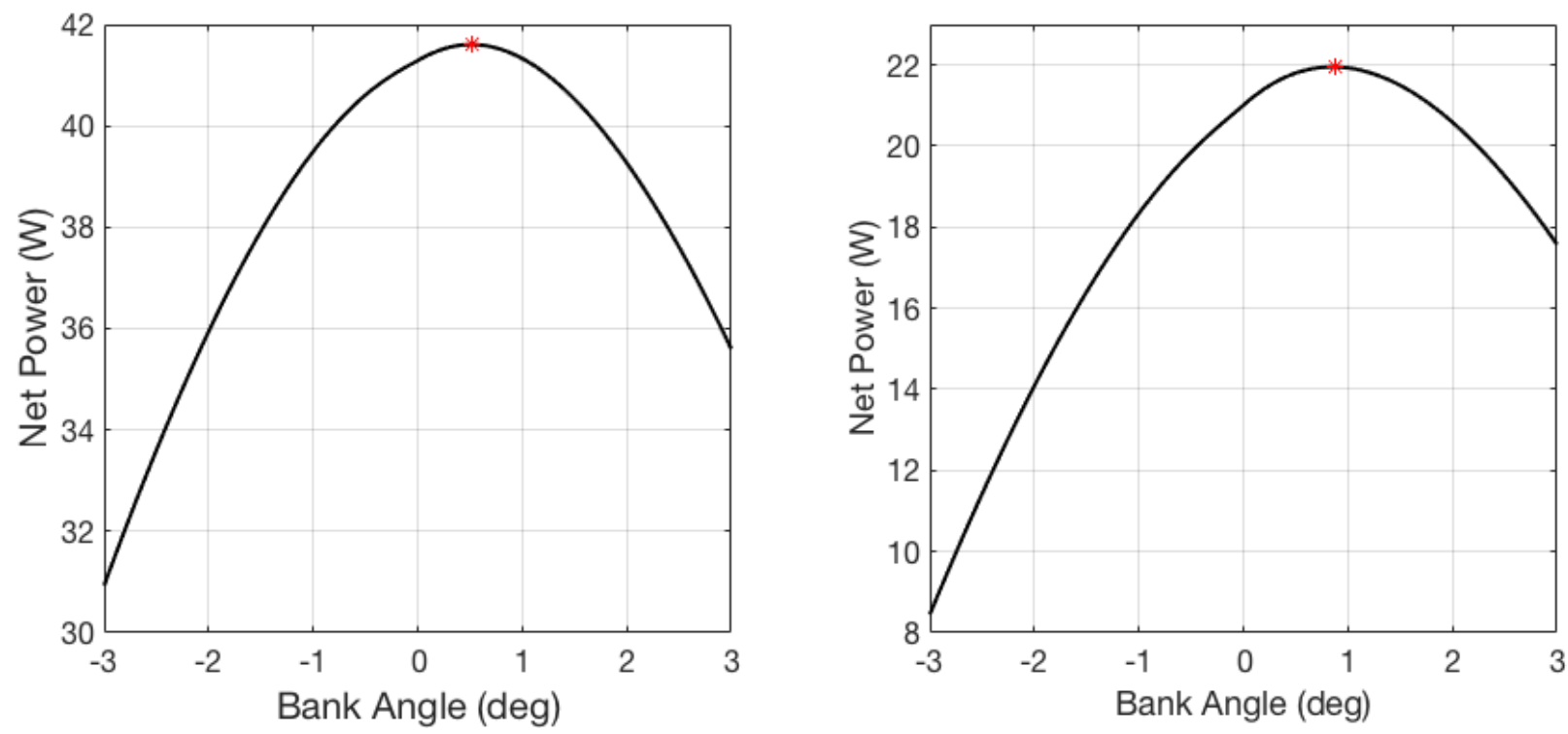

Figure 4.2: Net power for the Puma AE flying in San Luis Obispo, CA on the summer solstice at 12:00 PM (left) and 4:00 PM (right).

The sun moves through the sky from roughly east to west during the daytime, and for an aircraft heading of true north this means that bank angle for best net power should move from positive (roll right) to negative (roll left) throughout the day. Previously, all bank angles for best net power shown have been positive since the sun was always placed off of the right wing. The model was tested for this behavior and the affirmative results shown in Fig. 4.3. The flat portions of the plot at 0 bank angle 
show times where the sun elevation angle is negative, indicating that these are times pre-sunrise or post-sunset.

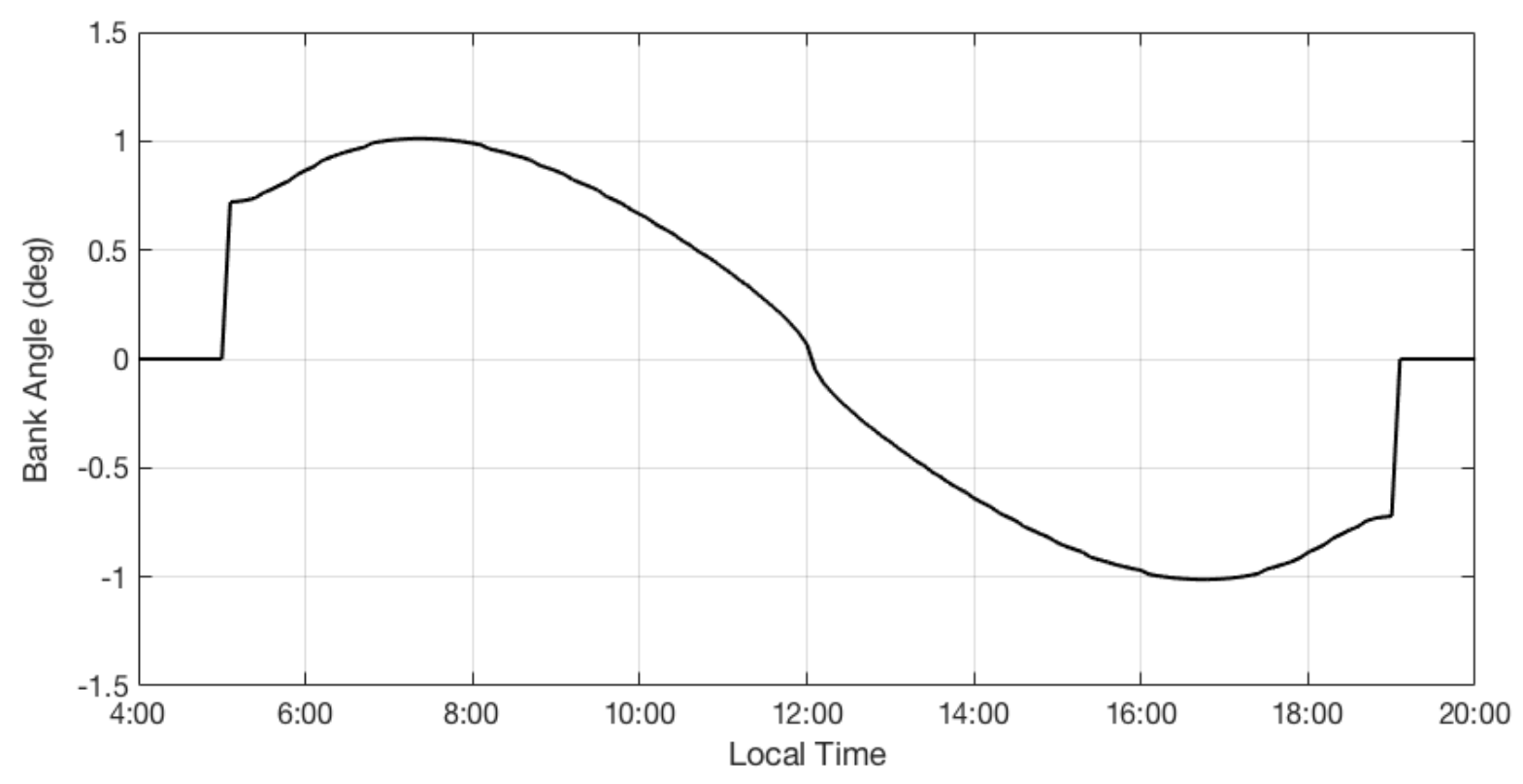

Figure 4.3: Bank angle for the Puma AE flying north asymmetrically in San Luis Obispo, CA through daytime on the summer solstice.

Next, the point performance model was interrogated for every day of the year at the previously selected times of 8:00 AM, 12:00 noon, and 4:00 PM. At each day/time combination, the bank angle for best net power was selected, and that net power was plotted in Fig. 4.4. Once again, aircraft heading was selected such that the sun was placed directly off the right wing of the aircraft, allowing the solar array to be rolled directly into the sun for best gains.

As expected, the highest net powers are seen in the summer months. For reference, the year 2016's summer solstice occurred June 20, which corresponds to a day number of 171. On the summer solstice, at local noon the sun is perceived to be directly above an observer on the Tropic of Cancer; instantaneously, this point is also the closest point to the sun on the Earth's surface. Since San Luis Obispo is north of the Tropic of Cancer, at noon on the summer solstice the sun's elevation angle will be at its maximum 


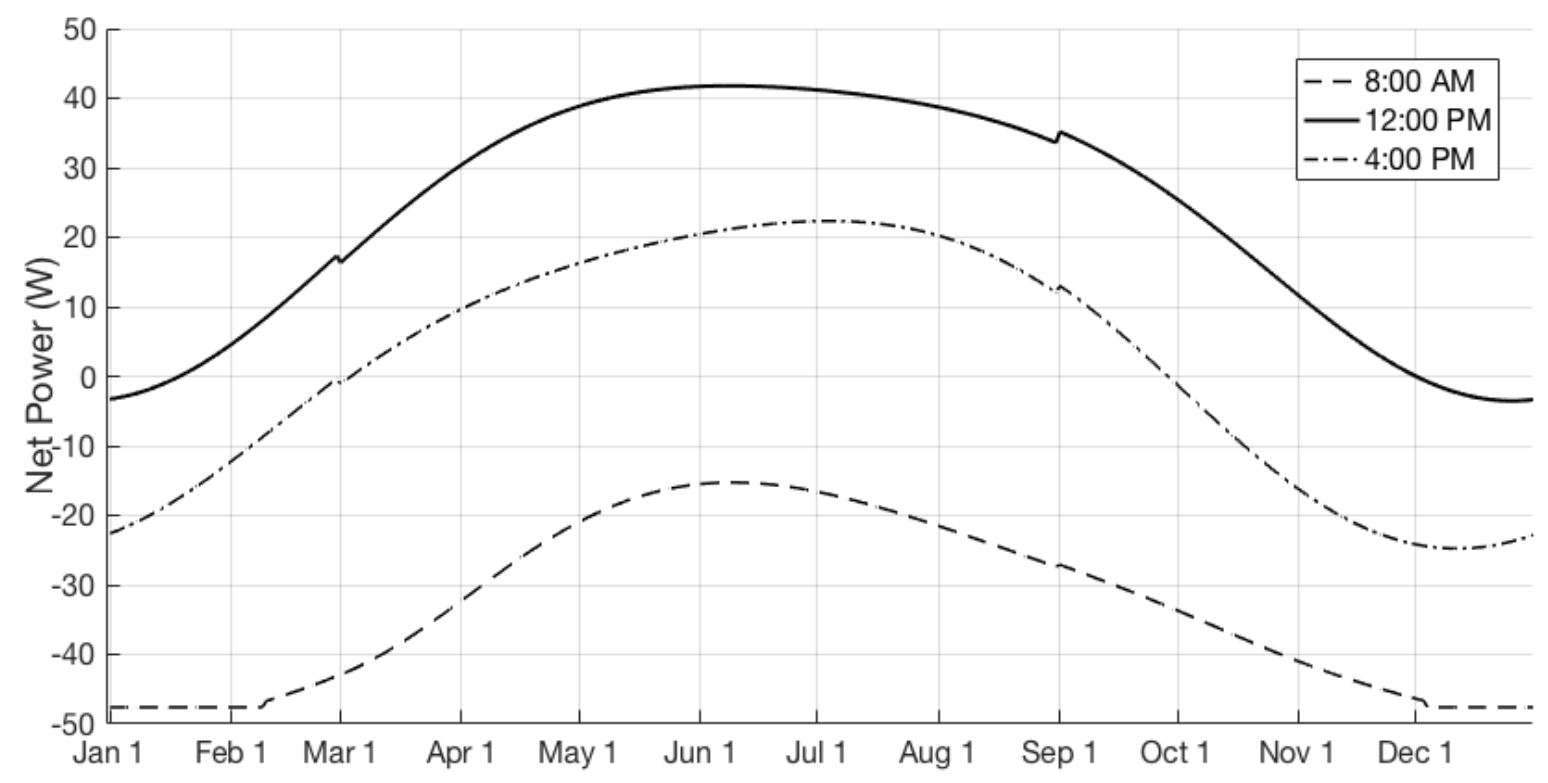

Figure 4.4: Net power for the Puma AE flying asymmetrically in San Luis Obispo, CA throughout an entire year for 3 different times of day.

for the year, but never directly overhead at an angle of 90 degrees.

Several spikes can be seen in the lines of Fig. 4.4. These spikes mark the changes in season, as modeled. Step changes in atmospheric transmittance coefficients (Eq. 3.12) with certain days of year associated with changes in season cause solar irradiance to also show step changes, which propagates through to the calculation of power available and finally net power. At 8:00 AM, for deep winter months a flat line in net power is shown. For those specific days, the sun is not yet above the horizon at 8:00 AM, and so power available is zero. Therefore, the model outputs the aircraft's power required for steady level flight as net power.

Another consideration of interest is what improvement in net power asymmetric flight provides over "symmetric" flight (i.e., coordinated flight at zero bank angle). Fig. 4.5 shows, for the summer solstice again in San Luis Obispo, net power versus time of day for an aircraft flying north. For fair comparison between asymmetric and symmetric flight, heading was not set in order to place the sun directly off of the wing. 


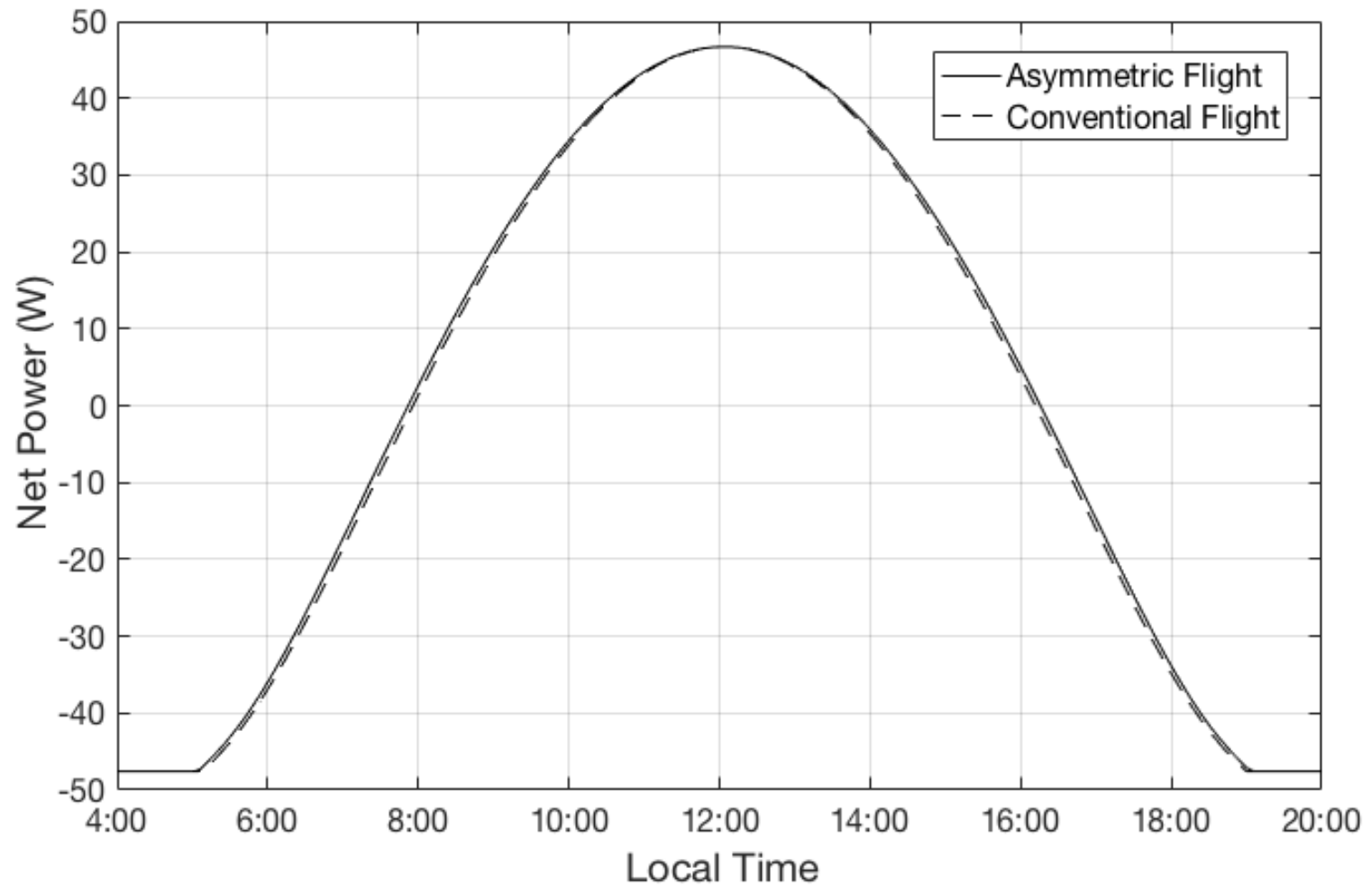

Figure 4.5: Net power for the Puma AE flying north in San Luis Obispo, CA on the summer solstice, both conventionally and asymmetrically.

It is shown in Fig. 4.5 that while there is indeed a benefit over conventional flight, it is very small. As expected, the improvement is more noticeable in the morning and afternoon hours due to a higher for improvement of net power at higher solar elevation angles. The flat portions at the beginning and end of the figure again show times where the sun is below the horizon and power available is zero. Although there is a very small noticeable improvement in a point performance context, over a mission that small improvement could become a more significant efficiency increase. Several data points from Fig. 4.5 are presented for comparison in Table 4.1.

Due to the interaction between aircraft heading and solar azimuth angle, the net power values shown in Table 4.1 will change with aircraft heading. At noon on the summer solstice in San Luis Obispo, azimuth angle is approximately 126 degrees, 
Table 4.1: Net power for asymmetric and conventional flight techniques flying north on the summer solstice in San Luis Obispo, CA.

\begin{tabular}{|l|c|c|c|}
\hline Local Time & Asymmetric $\mathbf{P}_{\text {net }}(\mathbf{W})$ & Conventional $_{\mathbf{P}_{\text {net }}}(\mathbf{W})$ & Improvement (\%) \\
\hline $08: 00$ & -17.2 & -18.5 & 5.4 \\
\hline $10: 00$ & 20.5 & 19.5 & 5.0 \\
\hline $12: 00$ & 43.4 & 43.2 & 0.5 \\
\hline $14: 00$ & 44.2 & 44.0 & 0.4 \\
\hline $16: 00$ & 22.5 & 21.6 & 4.2 \\
\hline $18: 00$ & -14.8 & 16.0 & 7.9 \\
\hline
\end{tabular}

placing the sun in the southeast. For asymmetric flight, it is desirable to fly headings where roll angle will have the most direct impact on improvements in incidence angle. It is conceivable, but out of scope for this work, that a flight path planner could be developed which takes into account the unique characteristics of asymmetric flight to plan both flight path and orientation for best overall aircraft efficiency.

While San Luis Obispo serves as a reasonable mid-latitude approximation for results in the United States, sensitivity of the model to geographic location should be examined. Latitude is the primary parameter of interest since changes in longitude only affect local time. In general, lower latitudes should show higher power available since the sun is typically higher in the sky at lower latitudes, leading to lower incidence angles. Figure 4.6 shows net power over daylight hours on the summer solstice at San Luis Obispo's longitude, but allowing latitude to vary across several important latitude lines. Aircraft heading was set so that the sun was off of the right wing in this case.

It is important that the summer solstice for the northern hemisphere is actually the winter solstice in the southern hemisphere, as the Tropic of Capricorn is at its furthest from the sun when the Tropic of Cancer is at its closest. For reference, the equator is located at 0 degrees latitude, the Tropic of Cancer at 23.5 degrees north and the Tropic of Capricorn at 23.5 degrees south (or -23.5 degrees). The Arctic and Antarctic circles 


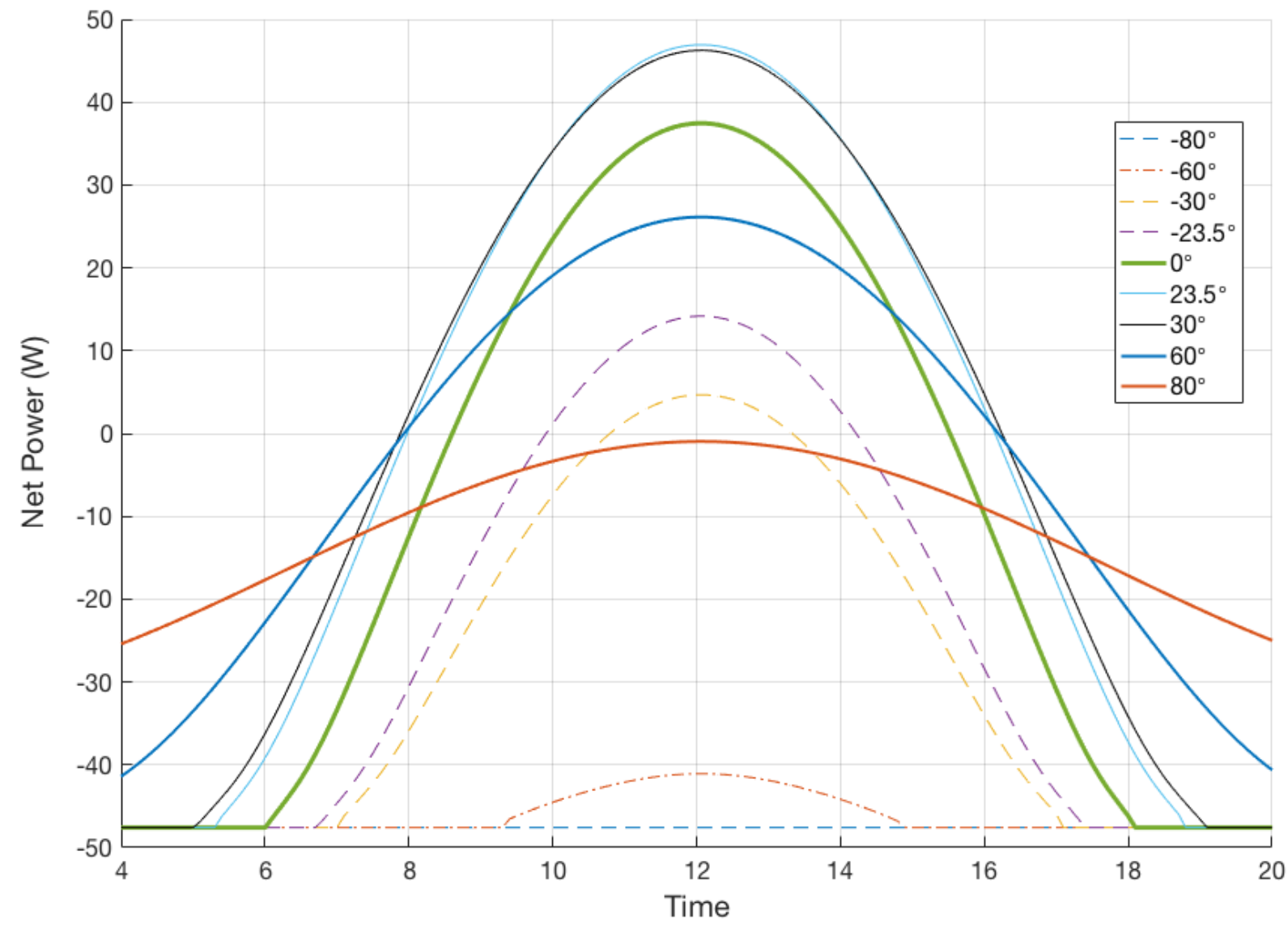

Figure 4.6: Net power for the Puma AE flying asymmetrically at San Luis Obispo's longitude on the northern hemisphere's summer solstice at varying latitudes.

begin at 66.5 degrees north and 66.5 degrees south, respectively, while the poles are located at 90 degrees north or south. A visualization of the geographic locations of Fig. 4.6's test points can be seen in Fig. 4.7.

Figure 4.6 shows trends as expected. On the northern hemisphere's summer solstice, extremely high southern latitudes see no sunlight at all, and daylight hours slowly increase from there. With no or almost no sunlight, net power is simply power required. Conversely, extremely high northern latitudes can see constant daylight hours in the summer months. While constant daylight is intriguing for perhaps an arctic exploration mission in the summer months, the low elevation angles seen at that high 


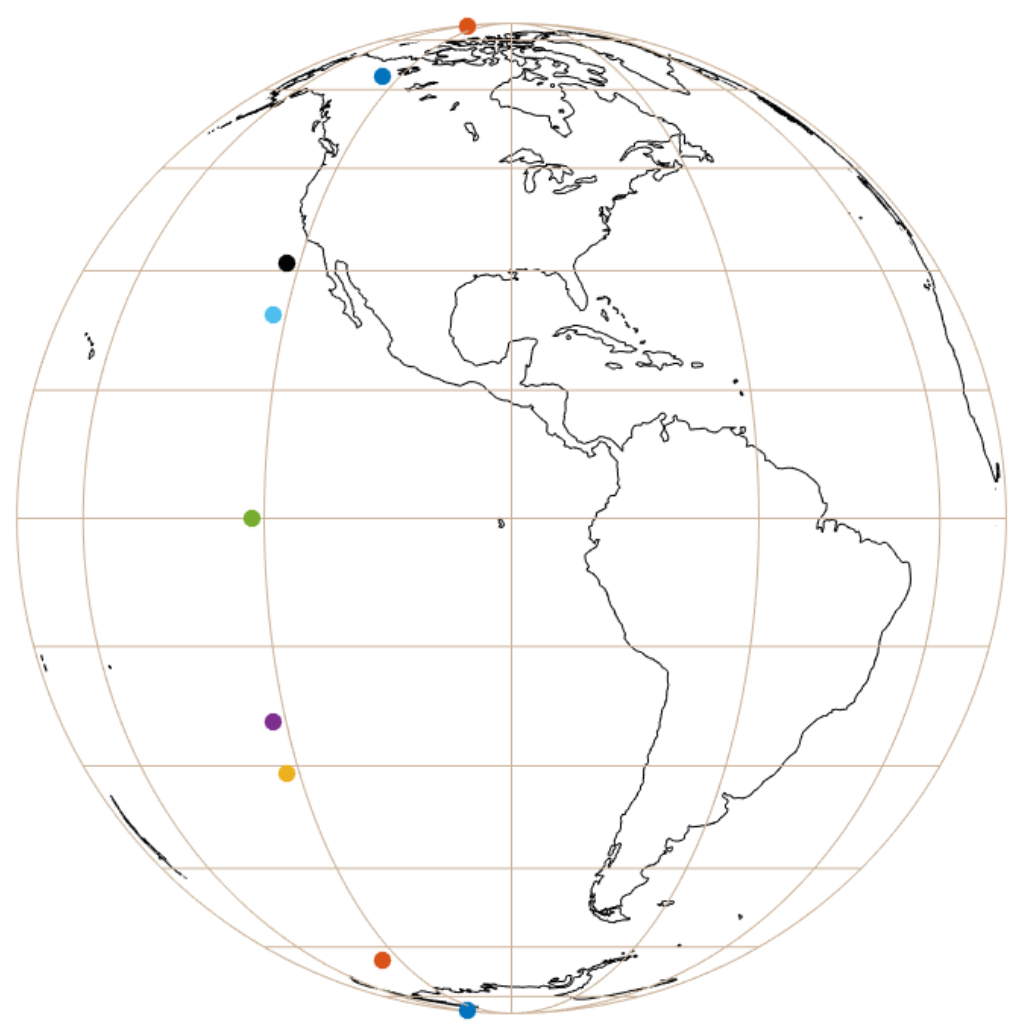

Figure 4.7: Geographic locations of the test points shown in Fig. 4.6. The selected longitude is that of San Luis Obispo, CA: $120.6^{\circ} \mathrm{W}$.

latitude still result in relatively poor net power. The opposite of course is true for the southern hemisphere's summer. One last point of interest can be seen by comparing the 23.5 degree north line (the Tropic of Cancer) and the 30 degree north line. As stated before, noon at the Tropic of Cancer on the northern hemisphere's summer solstice is the best case for solar radiation all year. However, the 30 degree north line shows almost identical behavior to that of the Tropic's line. Additionally, the best net power for San Luis Obispo (approximately 35 degrees north) at noon shown in Fig. 4.1 is about $42 \mathrm{~W}$, still quite close to the best net power shown at the Tropic of Cancer. This points to a sort of "sweet spot" for solar aircraft before large losses in net power are seen beginning around 60 degrees north. 


\subsection{Steady Turn Results}

The aerodynamics model also has the capability to model asymmetric flight in a steady turn. By assuming the aircraft can trim for a coordinated turn of a specified radius and corresponding bank angle, asymmetric flight can be performed around that coordinated turn bank angle to put the aircraft in an uncoordinated, asymmetric turn, and efficiency can be increased in some conditions since bank angle is already biased in one direction to allow the turn. That bank angle can then be manipulated further using asymmetric flight. This is one aspect that makes mission/orbit analysis interesting, because one side of an orbit will be biased towards the sun, but the other side will be roll-oriented away. As a result, there is an opportunity to investigate if different orbit shapes can lead to significant improvements in efficiency.

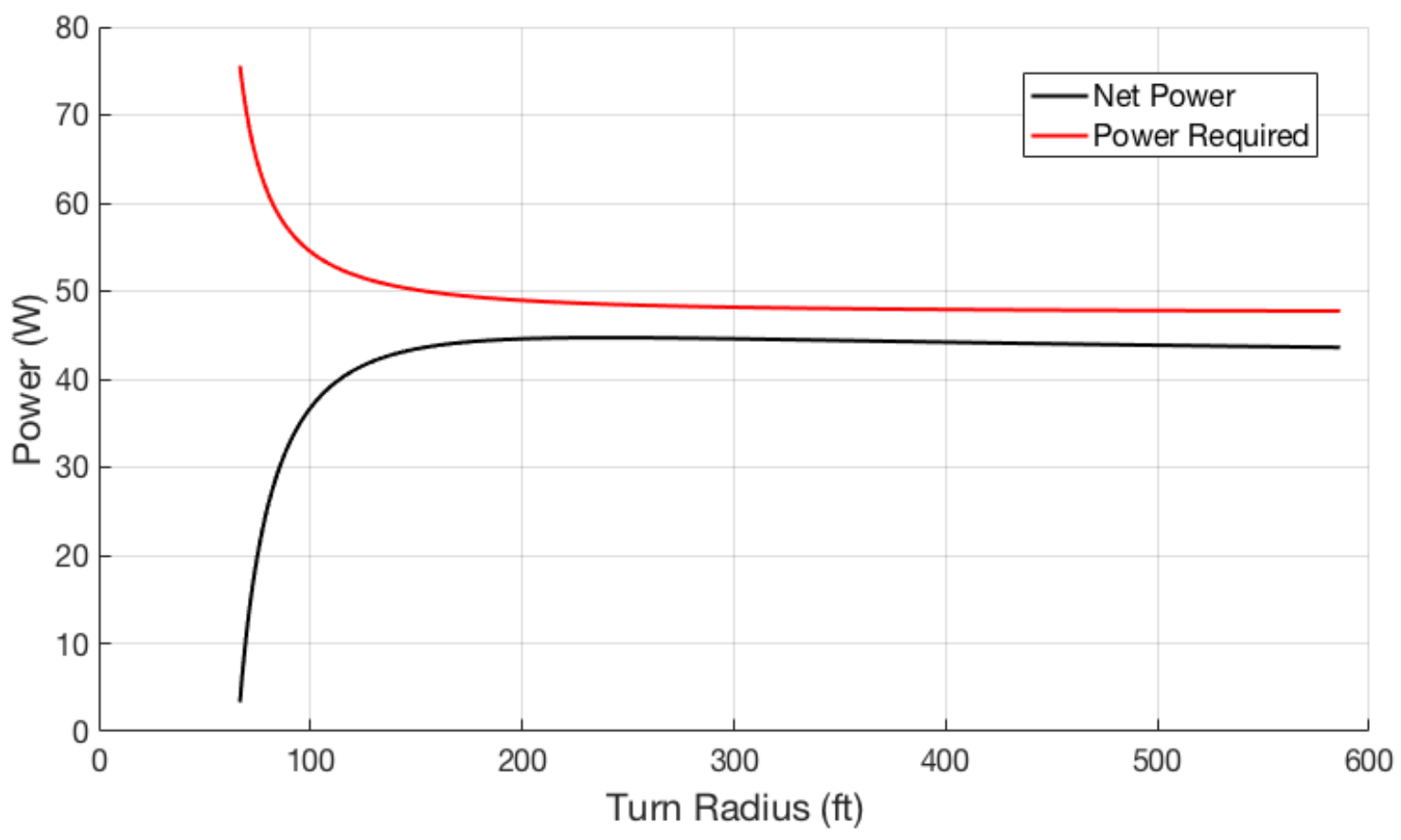

Figure 4.8: Net and required power for the Puma AE flying asymmetrically in San Luis Obispo, CA at noon on the summer solstice for various turn radii.

However, first the turn performance of the model should be evaluated in a point 
performance sense. As turn radius goes down, bank angle required to accomplish that turn increases. In order to maintain level flight, the aircraft must produce more lift by increasing angle of attack with bank angle. Increasing angle of attack has a strong effect on total drag and power required; meanwhile, any increase in power available depends entirely on the relative orientations of the aircraft and sun. Therefore, on a day and time such as noon on summer solstice where the sun is already high in the sky, one would expect little benefit, and eventually detriment, to net power from increasing turn radius. This is shown in Fig. 4.8, where the best asymmetric flight bank angle is being flown at each selected turn radius and the sun is placed off the right wing of the aircraft. Of course, turn radius is usually subject to mission requirements and any losses in net power are simply a price to be paid; however, there is potential for selection of turn radius based on efficiency.

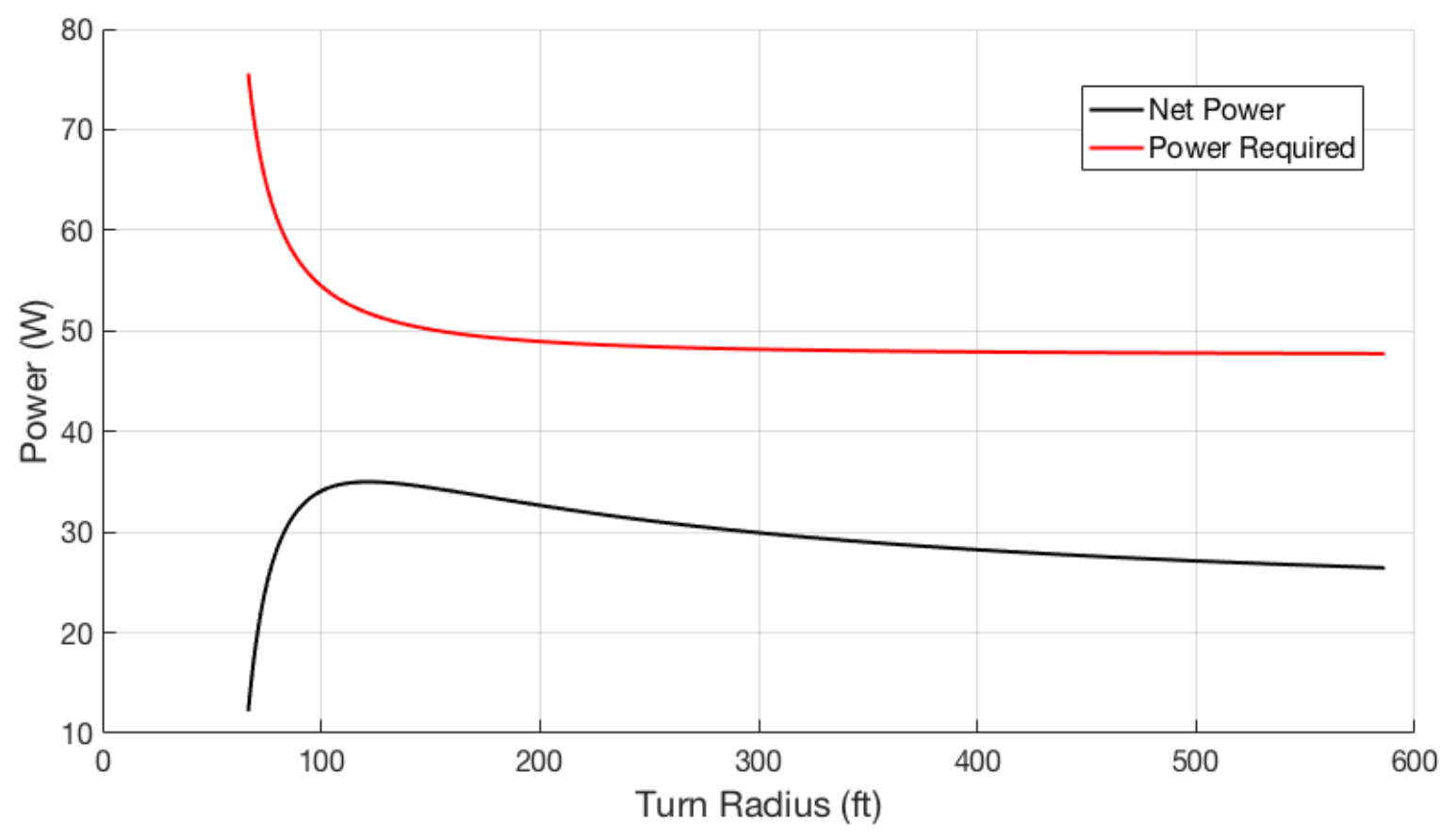

Figure 4.9: Net and required power for the Puma AE flying asymmetrically in San Luis Obispo, CA at 4:00 PM on the summer solstice for various turn radii.

Change the time to 4:00 PM, however, and one sees a significantly different picture 
in Fig. 4.9. Since the sun is now lower in the sky, there is a more dramatic effect on power available, and therefore net power, for banking into the sun. This is analogous to results seen in the previous section, but on a larger scale since bank angle can be manipulated much more by allowing the aircraft to turn.

With the point performance model behaving satisfactorily for both constant-heading flight and flight in a steady turn, attention can be turned to evaluating that model across various missions or orbit shapes. 


\section{Chapter 5}

\section{MISSION MODEL}

Mission performance can be extracted from a point performance model by evaluating a large series of points where input parameters are selected in order to approximate the desired mission. In this case, the objective is to evaluate the effects of asymmetric flight on the performance of solar sUAS as orbit platforms; therefore, a variety of orbit shapes will be treated as mission inputs. It is necessary to assume away dynamic maneuvers and assume step changes in aircraft and sun orientation, but this is acceptable with a sufficiently small time step. Capability was also built up to consider the effects of winds aloft on total aircraft efficiency.

\subsection{Definition of Aircraft Track}

Since the point performance model uses latitude, longitude, and altitude to define the aircraft's position on the Earth, the same approach must be used in advance by the mission model to define the aircraft's track. The Matlab Mapping Toolbox [14] was used to generate these sets of latitude, longitude, and altitude points. The toolbox has the ability to generate circles, ellipses, or arbitrary tracks on a reference sphere or ellipsoid. In this study, a spherical Earth was used, but for the relatively low altitudes and small sizes of orbits being computed here, it is likely that even assumption of a flat Earth would be acceptable. In this section, zero wind is assumed; the effects of wind will be added later.

With the desired track generated beforehand in terms of latitude and longitude, aircraft orientation must be modeled. Altitude and airspeed was held constant across 
all missions. First, the distance and bearing between two latitude/longitude points is calculated. Based on spherical mathematics, the Matlab Mapping Toolbox can again be used for this purpose to populate both distance and bearing for every leg of the mission. The resultant distance is the length of a great circle arc between the two points, and bearing is referenced to true north. For small distances, it is practical to treat this arc length as the length of a straight line between the two points, as shown in Fig. 5.1.

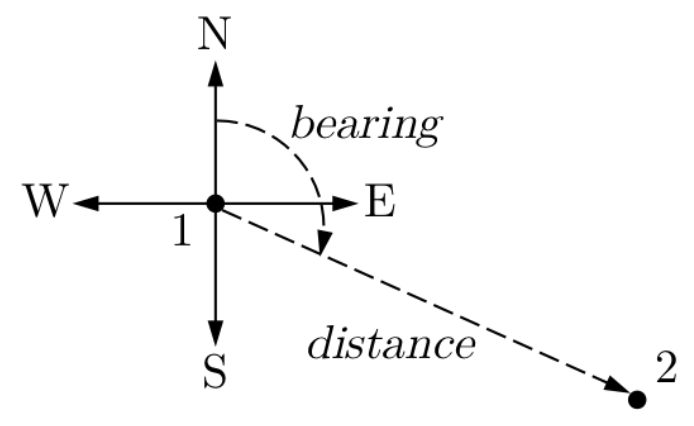

Figure 5.1: Visualization of bearing and distance for a sample mission leg between points 1 and 2 .

All that remains is to determine the turn radius for each leg so that the necessary bank angle for that turn radius can be computed and input to the point performance model. For an object in a steady turn, angular velocity $\omega$ is constant. Furthermore, angular velocity can be described as follows[15]:

$$
\begin{gathered}
\omega=\frac{V_{\infty}}{R} \\
\omega=\frac{d \psi}{d t}=\frac{\psi_{2}-\psi_{1}}{t}
\end{gathered}
$$

where $R$ is turn radius, $\psi$ is heading angle, and $t$ is the time step for the mission leg in question. Heading angle is set equal to the bearing for each leg; by inputting the 
entire track in advance, the required heading for the next leg, $\psi_{2}$, is easy to determine. The time step can be solved for by dividing the already-determined distance between points by airspeed, which is constant:

$$
t=\frac{\text { distance }}{V_{\infty}}
$$

By substituting (5.3) into (5.2) and setting the resultant equal to (5.1), turn radius can be solved for:

$$
R=\frac{\text { distance }}{\psi_{2}-\psi_{1}}
$$

With the necessary turn radius for each leg calculated, the necessary bank angle to accomplish that turn can now be determined. This is the bank angle around which asymmetric flight can be performed as in Sec. 4.2. Steady turn performance theory from [15] can again be looked to:

$$
\begin{aligned}
& \cos (\phi)=\frac{1}{n}=\frac{W}{L} \\
& R=\frac{W}{L} \frac{V_{\infty}^{2}}{g \sin (\phi)}
\end{aligned}
$$

Where $n$ is load factor and $g$ is acceleration due to gravity. Equation (5.5) can be substituted into (5.6) and solved for $\phi$ :

$$
\phi=\tan ^{-1}\left(\frac{V_{\infty}^{2}}{R g}\right)
$$

It is worth noting that when using compass bearings to describe aircraft heading, certain logic must be developed to compute the difference in headings between two 
mission points, as required by Eq. (5.2). In this model, the aircraft should always turn through the smallest heading change possible, and the signs of the output bank angle and heading change must correspond. As defined here, a positive bank angle results in a right turn, which will cause an increase in heading angle. In some cases, where the difference between headings is less than 180 degrees and does not go over the break from 359 to 0 degrees, it is sufficient to simply subtract the two headings. Additionally, turn radius, as defined in Eq. (5.4), requires that the heading change in the denominator be defined positive for the resulting turn radius to be positive and therefore have physical meaning. Simultaneously, however, the sign of the required heading change must be preserved in order to determine the correct bank angle. Consider Fig. 5.2, where logic must be used to redefine the calculated change in heading and bank angle because simple subtraction of heading results in a difference of greater than 180 degrees.

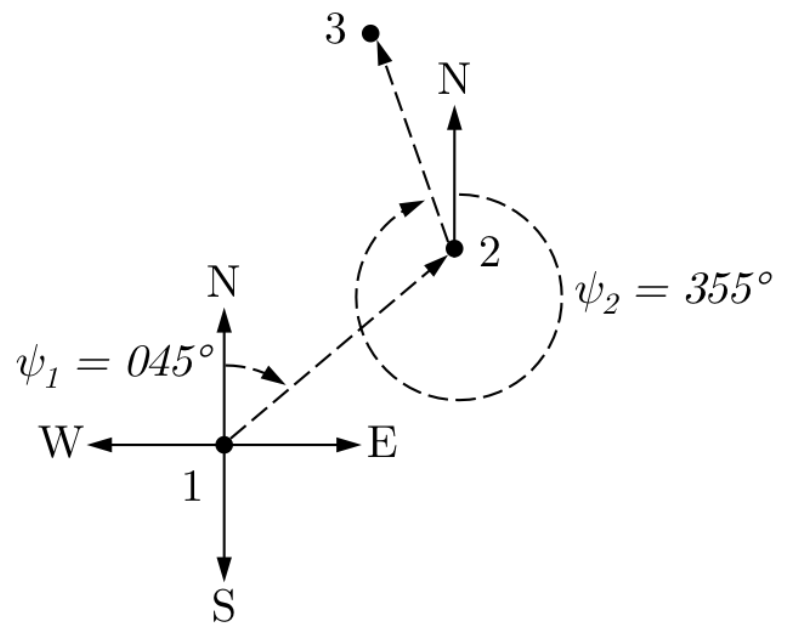

Figure 5.2: Mission case where simple subtraction of heading angles is insufficient.

The approach taken in this work to correct for situations such as that shown in Fig. 5.2 is summarized below. The following is used if $\left|\psi_{1}-\psi_{2}\right|$ is greater than 180 degrees: 


$$
\begin{aligned}
& \psi_{1}= \begin{cases}\psi_{1} & \text { if } \psi_{2} \leq 180 \\
\psi_{1}+360 & \text { if } \psi_{2}>180\end{cases} \\
& \psi_{2}= \begin{cases}\psi_{2} & \text { if } \psi_{1} \leq 180 \\
\psi_{2}+360 & \text { if } \psi_{1}>180\end{cases}
\end{aligned}
$$

Equations (5.8) and (5.9) simply add 360 degrees to the lesser heading angle if the difference between the two is greater than 180 degrees, which will generally only occur when going over the break between 359 and 0 degrees. This logic results in the correct heading difference being used. One last correction is required to ensure that the aircraft turns the correct way. If the "corrected" heading difference (after applying the logic above) is positive, the aircraft should turn left by applying a negative bank angle.

$$
\phi= \begin{cases}\phi & \text { if } \psi_{1}-\psi_{2}<0 \\ -\phi & \text { if } \psi_{1}-\psi_{2}>0\end{cases}
$$

With time step, latitude, longitude, heading, and bank angle determined, the point performance model can be called on to determine net power at every mission point, which can be used later on to find net energy expended over the course of a mission.

\subsection{Adding Winds Aloft}

Winds can have a large effect on an aircraft's performance, and capability was developed to model the effects of a wind of constant input speed and direction. Since the aircraft's track without wind (analogous to ground track) was already determined in the previous section, and the goal is to hold that ground track despite an input wind 
vector, the only changes necessary are to update the aircraft's heading and time step for each point. The aircraft's heading is manipulated by the model in order to maintain the direction of the desired ground track, while the aircraft's ground speed (and the resultant time step) is allowed to change since airspeed is held constant.

Vector arithmetic is the method used to determine the two unknowns, ground speed $G$ and heading $\psi$. A sample case is shown in Fig. 5.3 for notional mission points 1 and 2, with input wind speed $W$, wind direction $\theta_{W}$, and airspeed $A$ of 24 knots. Bearing $\theta_{G}$ is found in the previous section.

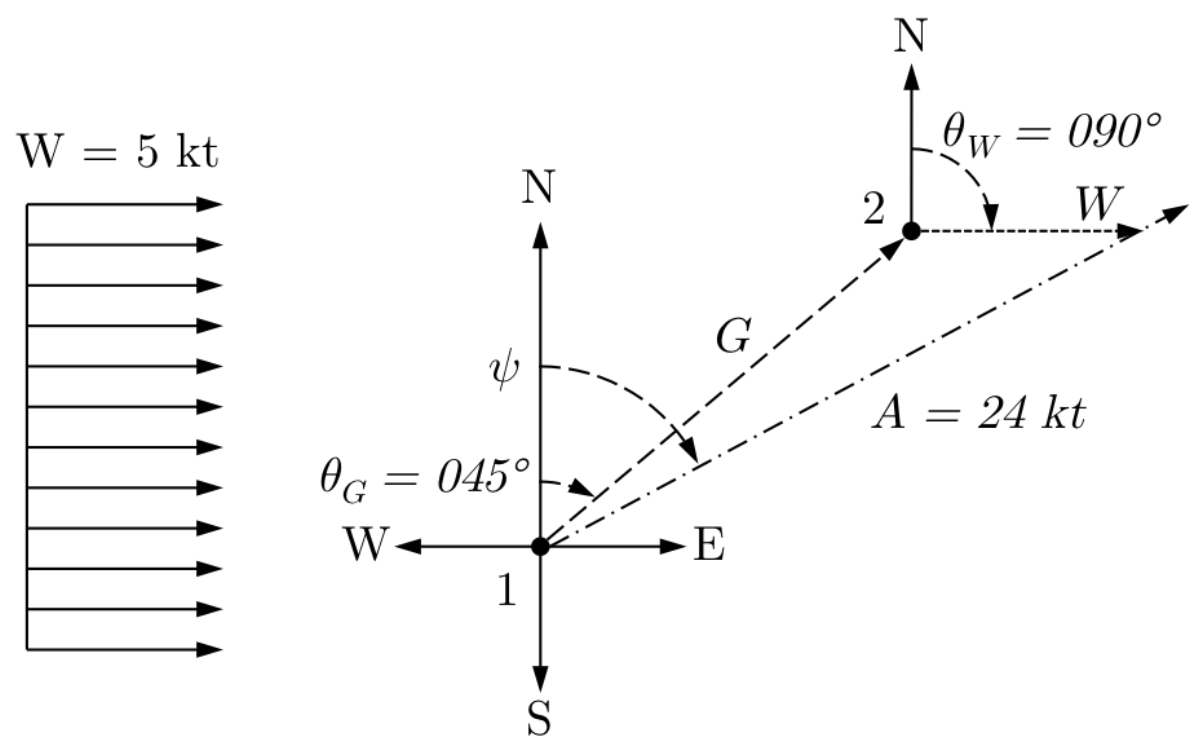

Figure 5.3: Example case showing vectors used to model effects of winds aloft.

Two equations can be generated via vector summation to solve for the two unknowns in this case:

$$
\begin{aligned}
& A \cos (\psi)+W \cos \left(\theta_{W}\right)=G \cos \left(\theta_{G}\right) \\
& A \sin (\psi)+W \sin \left(\theta_{W}\right)=G \sin \left(\theta_{G}\right)
\end{aligned}
$$


Again because of the peculiarities of working with compass headings and trigonometric functions, care must be taken to ensure that resultant values have the correct signs and magnitudes. In order to get around some of the difficulties associated with solving the above set of equations, an iterative method was designed such that, for a guess of aircraft heading, the resultant ground track bearing was calculated and compared to the known bearing from point 1 to point 2 . Once the aircraft heading is converged on, it is simple to solve for ground speed by adding the now fully-determined air and wind vectors.

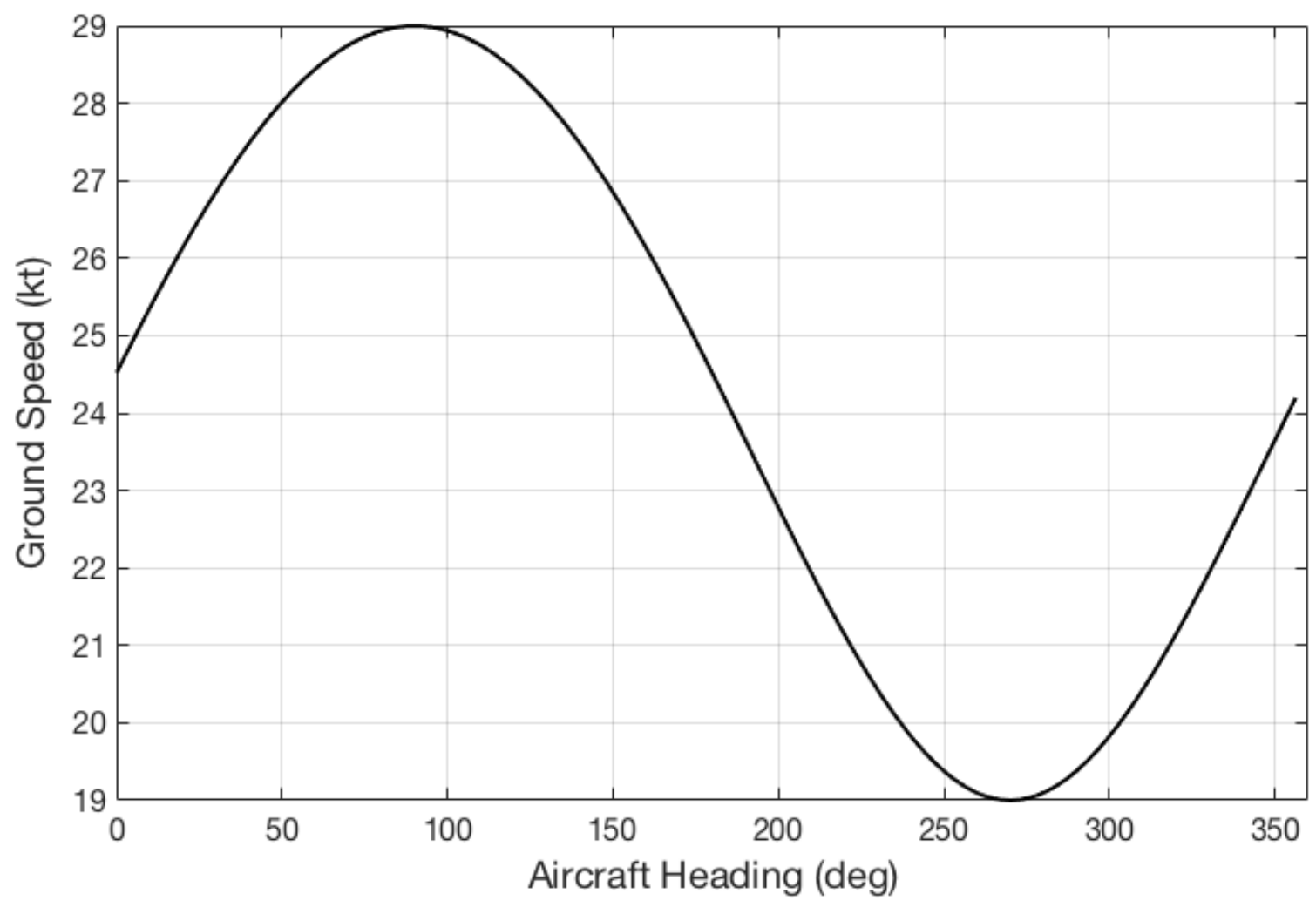

Figure 5.4: Effects of a 5-knot wind from 270 degrees on ground speed for a circular orbit.

In order to test the results of the mission model with wind effects now added, a test mission consisting of a single circular orbit around a point was run for various (constant) wind speeds and directions. For a circular orbit with zero wind, ground 
speed would be constant and equal to airspeed, which is shown in the model. However, by flying a circular orbit, a heading change is introduced and ground speed will vary with heading as the aircraft turns through that circle. An example of this variation in ground speed is shown in Fig. 5.4 for a circular orbit of radius $200 \mathrm{ft}$ with winds of 5 knots from heading 270 degrees. One check for model validity here is that when the aircraft is flying directly into the wind, heading 270 , the ground speed is 19 knots, which is the airspeed of 24 knots minus the wind speed of 5 knots. If wind direction and strength is changed while holding the desired orbit ground track constant, the curve should both shift left to right and change shape, as shown in Fig. 5.5 for a wind of 10 knots from 180 degrees:

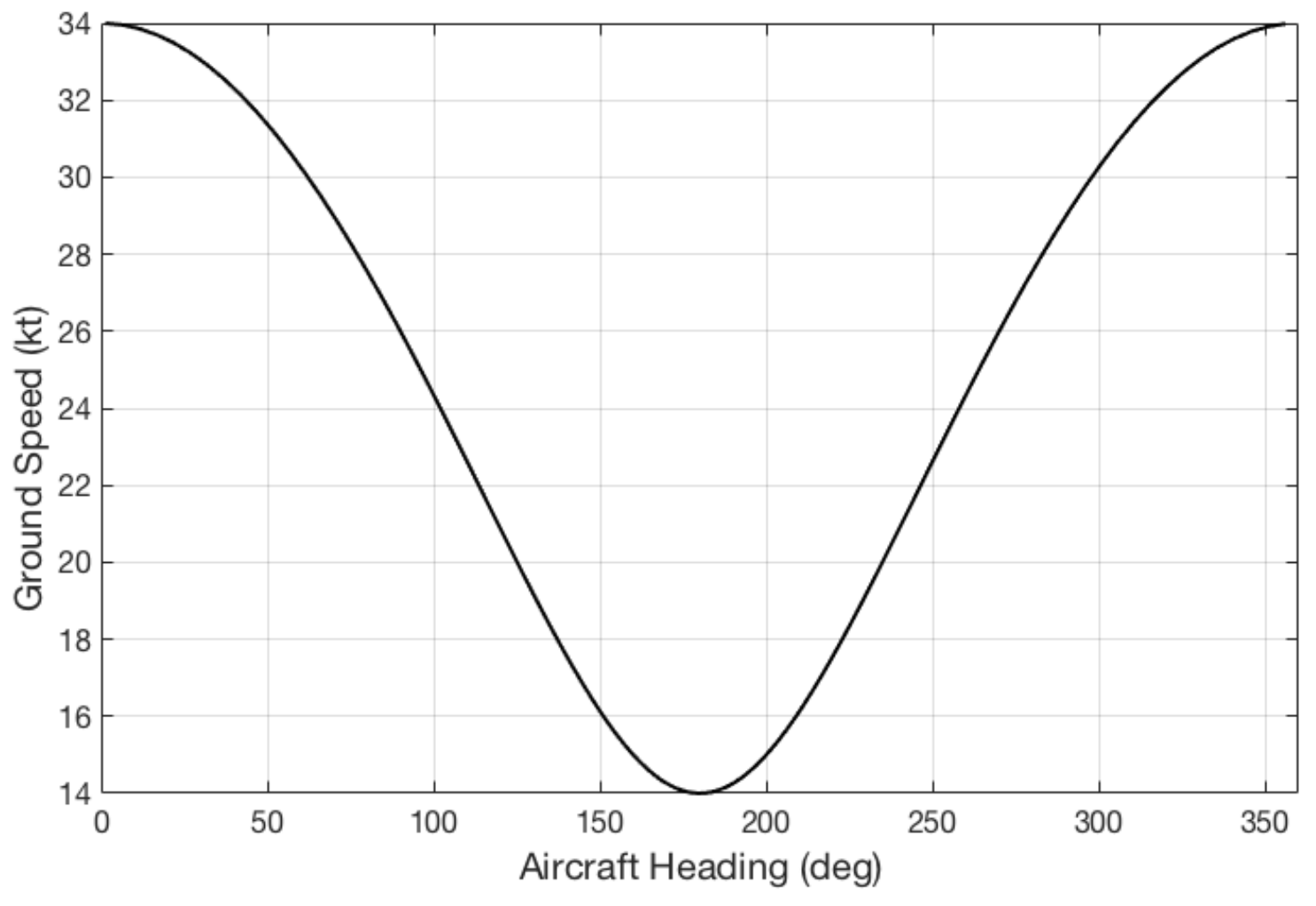

Figure 5.5: Effects of a 10-knot wind from 180 degrees on ground speed for a circular orbit.

When flying with the wind in Fig. 5.5, ground speed is modeled as 34 knots, which 
once again is the airspeed added to the wind speed and is as expected. With indication that aircraft dynamics are being modeled correctly in the presence of a constant wind vector, mission analysis can proceed. If a wind vector is input to the model, there is only one point performance input that needs to be changed from Sec. 5.1, heading angle, and one mission model parameter: time step. Time step (Eq. 5.3) must be re-computed with the newly-calculated ground speed, which changes through every mission leg. 


\section{Chapter 6}

\section{MISSION RESULTS}

While the asymmetric flight technique has shown some potential in point performance, mission performance should also be examined. Small efficiency gains at particular points could have their effects magnified, or lessened, by particular mission characteristics such as flight path, time of day, and winds aloft. Since small UAS are commonly used as reconnaissance platforms, orbits over a ground target are of particular interest, and it is conceivable that due to the unique nature of solar aircraft and asymmetric flight, there may be efficiency gains from manipulating orbit shape.

While optimization techniques could be used to find the optimal best orbit flight path for each specific set of possible inputs, the scope of this work is to define a few candidate orbit shapes and compute the net energy balance for each shape in an attempt to develop an understanding or strategy for improving aircraft efficiency. While net power was considered in point performance, for a mission the total net energy balance can instead be calculated and is indicative of overall vehicle performance. For simplicity's sake, the first orbit flight path of interest is a circular orbit of varying radius. Elliptical orbits are also considered.

Another application for sUAS is land survey where a vehicle is flown in a grid pattern over an area of interest, with grid spacing and number of legs usually corresponding to swath width of an onboard, downward-looking sensor. The effects of grid orientation on net energy balance with variation in sun position and winds are examined. 


\subsection{Circular Orbit Performance}

In mission analysis, the key performance parameter is the net energy balance across the selected mission. Similar to net power, a higher net energy is better, and a negative net energy indicates that energy would have to be expended from flight batteries, while a positive net energy indicates that flight batteries could actually be charged. The same model inputs as in the previous sections are required, in addition to the new inputs of orbit radius and wind strength and direction. Since effects of geographic location were already discussed and likely to be similar to those effects in point performance, the inputs to be varied here are time of day, day of year, orbit radius and winds.

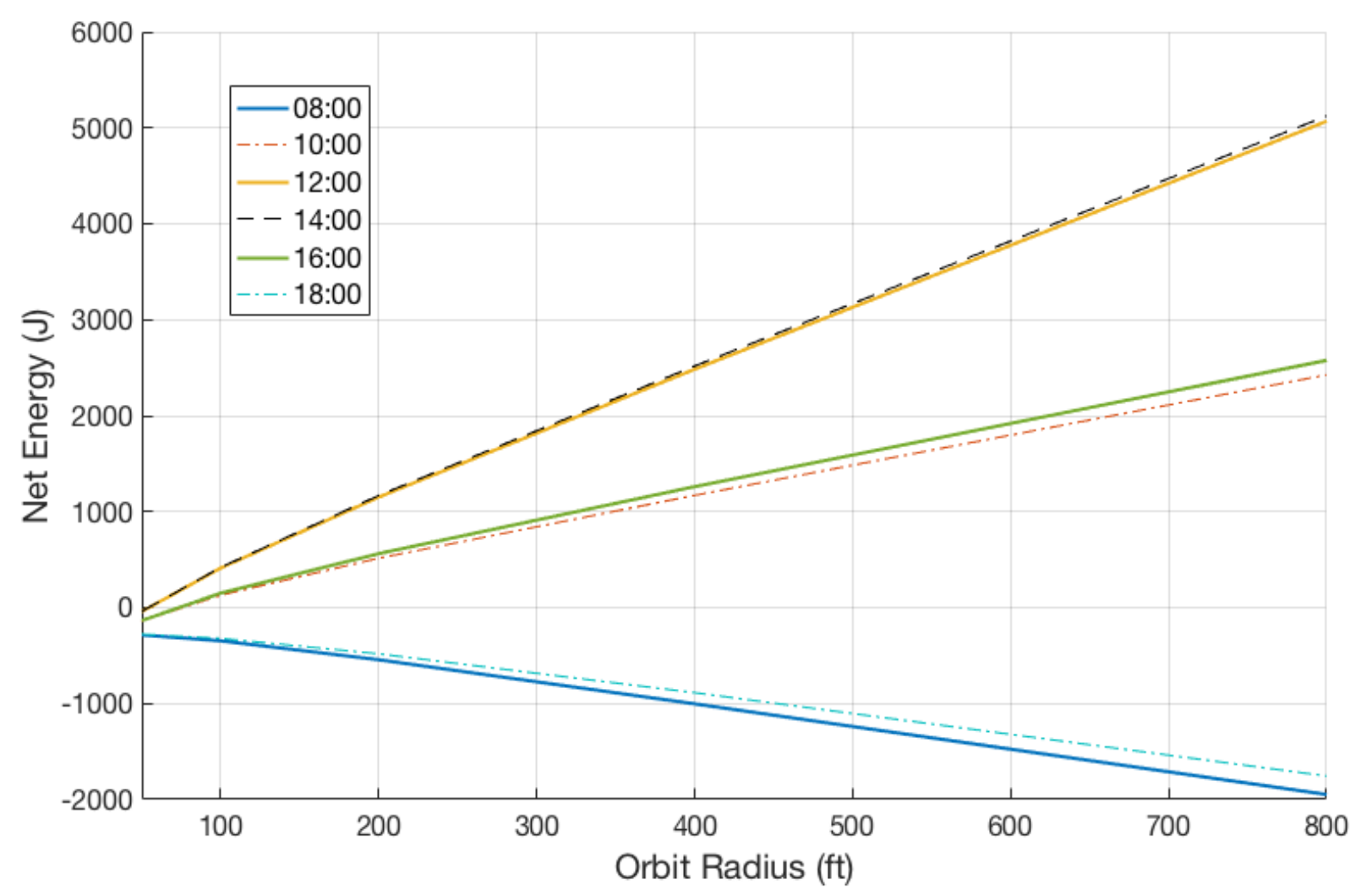

Figure 6.1: Net Energy vs. Orbit Radius for various times of day on the summer solstice in San Luis Obispo, CA.

Different trends are seen in net energy for different times of day in Fig. 6.1. In the 
morning and afternoon, the sun is low in the sky, and tighter orbits, corresponding with higher bank angles, will show more improvement in energy collection. Towards the middle of the day, asymmetric flight pays off less and banking for a turn actually makes the incidence angle worse. It makes sense then, that in the morning lower orbit radii show better net energies than at noon, while higher orbit radii show better performance towards the middle of the day.

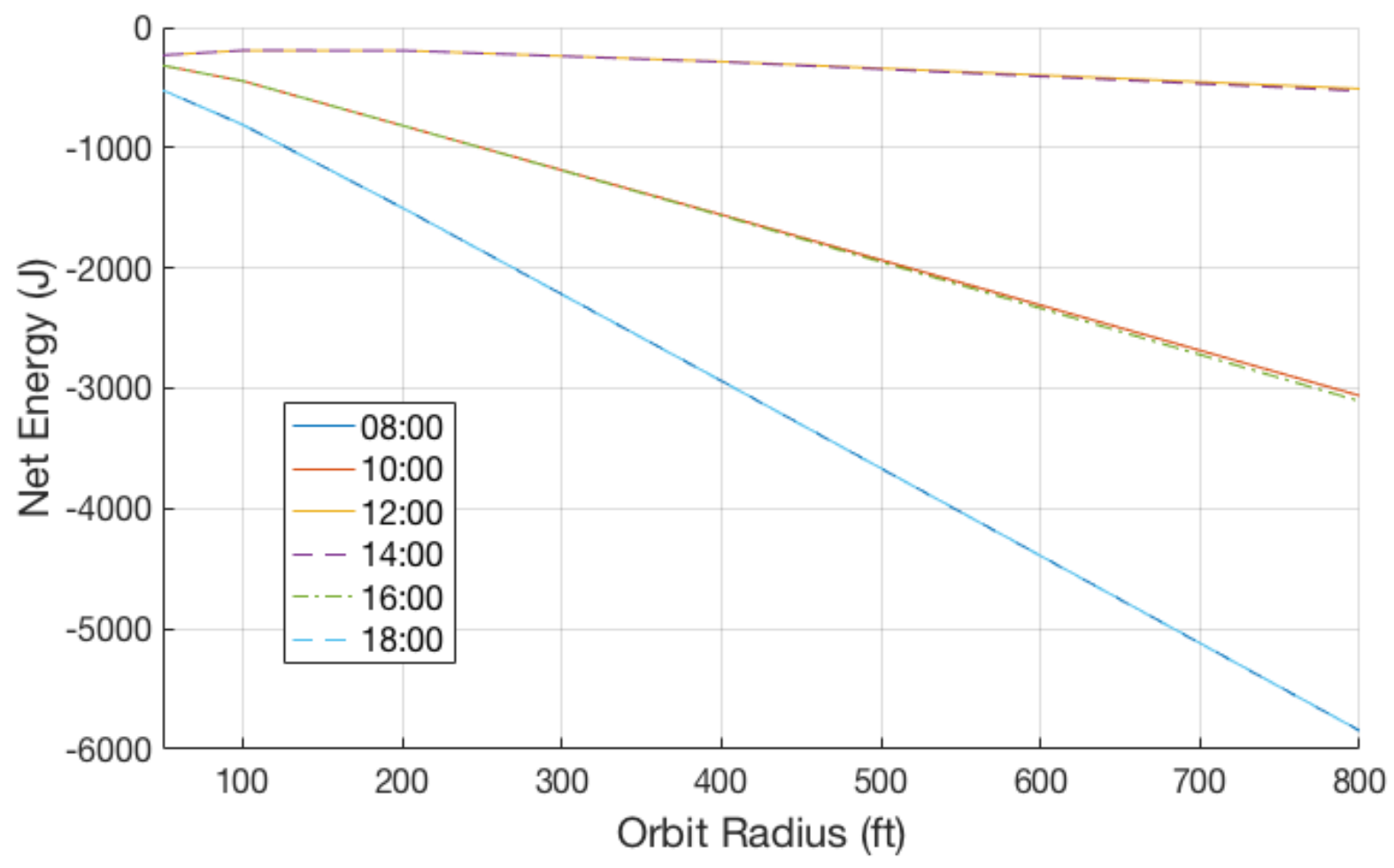

Figure 6.2: Net Energy vs. Orbit Radius for various times of day on the winter solstice in San Luis Obispo, CA.

On the winter solstice, the sun is lower throughout the day and net energy should decrease across the board, shown in Fig. 6.2. It is interesting to see that on the winter solstice, lower orbit radii (and higher bank angles) are uniformly better in terms of net energy, as compared to the summer solstice where lower orbit radii are only better for early morning and late afternoon.

While Figs. 6.1 and 6.2 are interesting in that they show a break point in times 
of day where orbit strategy should change between large and small orbit radii, they do not tell the whole story in terms of orbit efficiency by displaying simply the raw net energy output. If a particular orbit has a positive net energy, by making the orbit radius larger, the orbit takes longer and there is more time to collect energy, causing the behavior shown where net energy simply increases with orbit radius. In order for a fair comparison, a metric that is normalized by the total time taken to accomplish that orbit should be used. Average power can be computed by dividing the net energy by the total orbit time, and shows a more accurate picture of orbit efficiency.

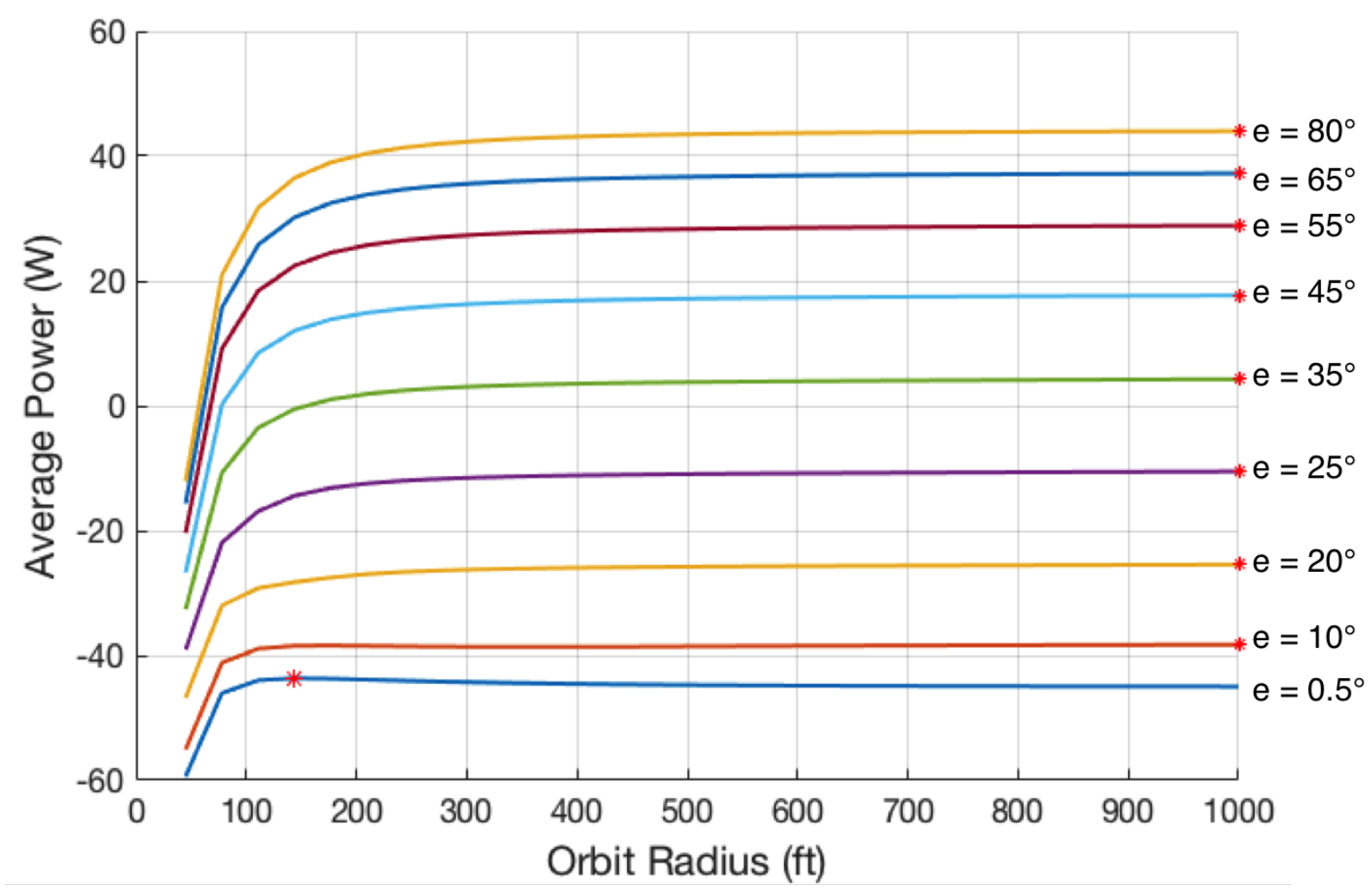

Figure 6.3: Average Power vs. Orbit Radius for asymmetric flight over various solar elevation angles on the summer solstice in San Luis Obispo, CA.

For both the summer and winter solstice, results show that there is a break point in sun elevation angle; for very low elevation angles, small orbits show better average power, as shown by the red asterisks. Note that the maximum and minimum elevation 
angles seen in Figs. 6.3 and 6.4 are set by day of year and geographic location. Past approximately 5 degrees elevation angle, large orbits become more beneficial, and the red asterisks for best average power are pushed off the end of the plot. For the Puma's flight condition, a 1000-ft orbit radius corresponds with a turn bank angle of about 3 degrees, already very low. Once again, higher average powers are seen in the summer than in the winter, as should be expected. It seems clear that the increased power requirements due to bank angle dominate over the increase in power available due to improved incidence angles at those high bank angles; reference Fig. 4.8, whose shape is very similar to the more comprehensive plots shown in this section. Based on these results, it appears that flying as large an orbit as possible is better in all cases except the extreme early morning.

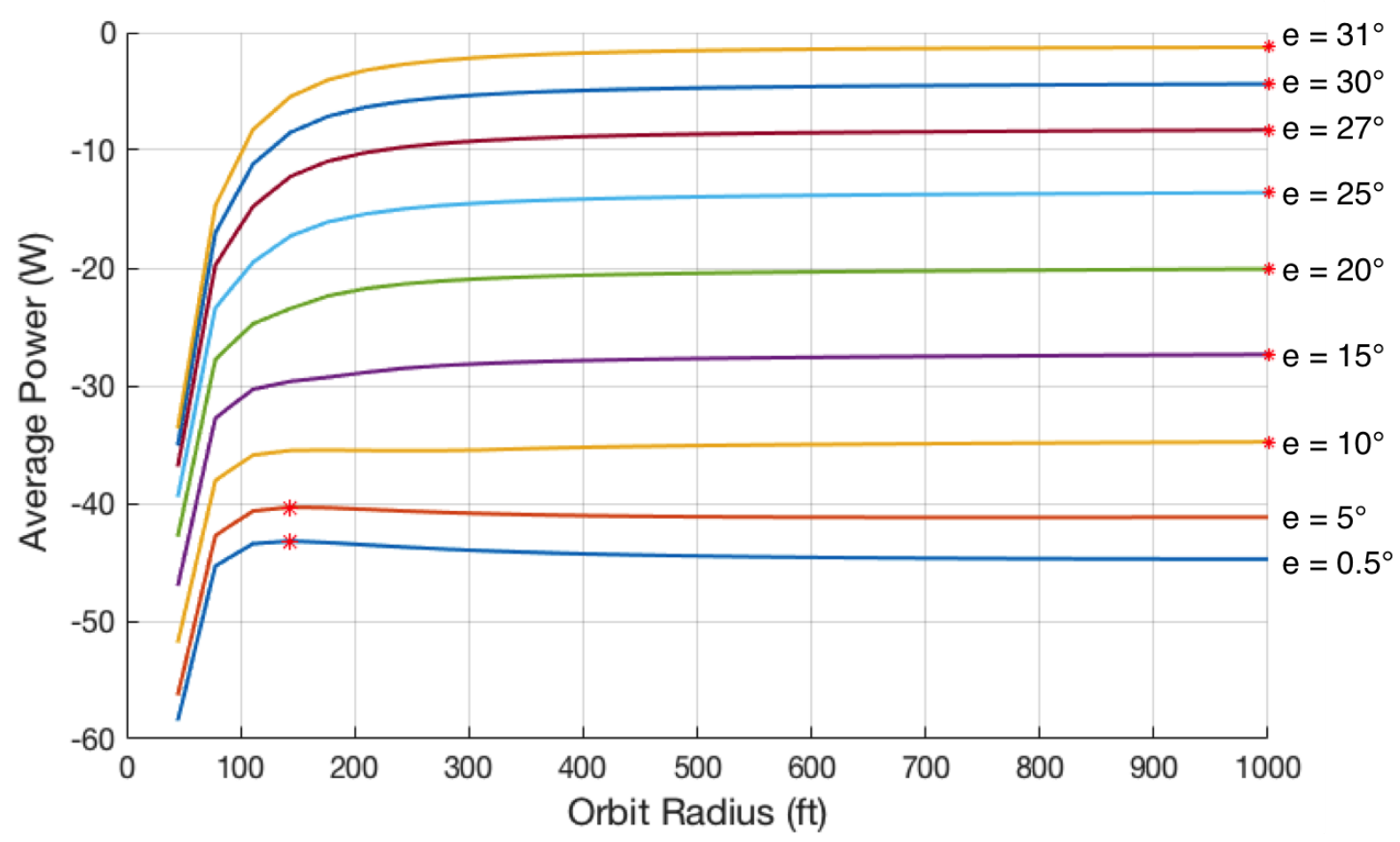

Figure 6.4: Average Power vs. Orbit Radius for asymmetric flight over various solar elevation angles on the winter solstice in San Luis Obispo, CA.

For interest, a plot of the same results, showing much the same conclusions, is 
included for the summer equinox in Fig. 6.5. The summer (or winter) equinox is the time of year when day and night are approximately equal world-wide, and is a middling case for solar radiation.

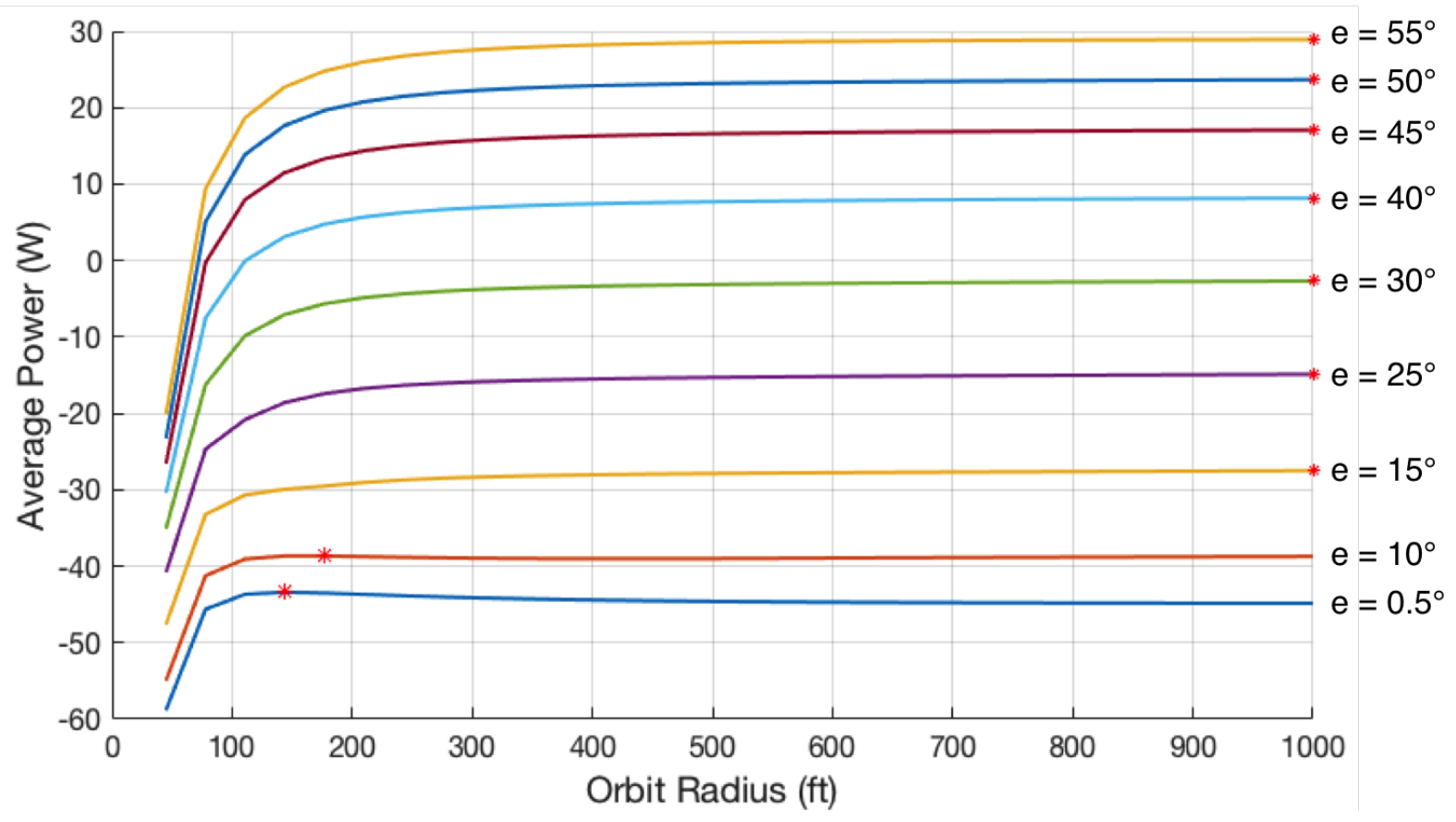

Figure 6.5: Average Power vs. Orbit Radius for asymmetric flight over various solar elevation angles on the summer equinox in San Luis Obispo, CA.

Another topic to be considered is the actual benefit of asymmetric flight as compared to conventional flight across these various orbit shapes. While the previous discussion centered on the effect of orbit radius on orbits being flown with asymmetric flight, now one can compare efficiencies across the same orbits being flown both asymmetrically and conventionally for several times of day in Table 6.1.

Before the sun reaches high elevation angles, asymmetric flight shows improvements in the order of 3-6\% over conventional flight, at least for circular orbits. While this is a relatively small improvement, it is nonetheless a potential efficiency gain that requires only operating the aircraft in a different way than normal. 
Table 6.1: Comparison of Net Energy for two different orbit radii throughout the summer solstice in San Luis Obispo, CA.

\begin{tabular}{|c|c|c|c|c|}
\hline Time & $\begin{array}{c}\text { Orbit } \\
\text { Radius } \\
(\mathbf{f t})\end{array}$ & $\begin{array}{c}\text { Conventional } \\
\text { Flight } \\
\text { Net Energy }\end{array}$ & $\begin{array}{c}\text { Asymmetric } \\
\text { Flight } \\
\text { Net Energy }\end{array}$ & $\begin{array}{c}\% \\
\text { Improvement }\end{array}$ \\
\hline $08: 00$ & 200 & $-583 \mathrm{~J}$ & $-546 \mathrm{~J}$ & $6.3 \%$ \\
& 400 & $-1065 \mathrm{~J}$ & $-1014 \mathrm{~J}$ & $4.8 \%$ \\
\hline $10: 00$ & 200 & $483 \mathrm{~J}$ & $513 \mathrm{~J}$ & $6.1 \%$ \\
& 400 & $1121 \mathrm{~J}$ & $1162 \mathrm{~J}$ & $3.6 \%$ \\
\hline $12: 00$ & 200 & $1140 \mathrm{~J}$ & $1150 \mathrm{~J}$ & $0.93 \%$ \\
& 400 & $2468 \mathrm{~J}$ & $2481 \mathrm{~J}$ & $0.55 \%$ \\
\hline
\end{tabular}

\subsubsection{Winds on Circular Orbits}

To study the effects of winds aloft on circular orbit performance, an arbitrary situation can be used where the sun position is set such that the azimuth angle is directly East, 090 degrees, with an elevation of 45 degrees. This will allow determination of the effects of various winds on orbit efficiency in isolation.

If winds are out of the north, in order to maintain a circular ground track, the aircraft will fly through the air a shorter downwind leg and a longer upwind leg, as shown in Fig. 6.6.

It would appear that Fig. 6.6 shows a case of beneficial interaction between wind direction and sun location, since this situation places the sun off the aircraft's wing when the aircraft's right (or left, depending on direction of orbit) wing is banked towards the sun longer than it would be if it were flying a circular orbit without wind. While it is true that in a larger radius turn the relative bank angle towards the sun is less, the results shown in the previous section point towards larger turn radii being better in terms of net power due to the less power required to execute that turn at constant altitude. However, were the sun in the west, this would be a detrimental interaction for the same reasons. In order to more fully investigate these interactions, wind direction 


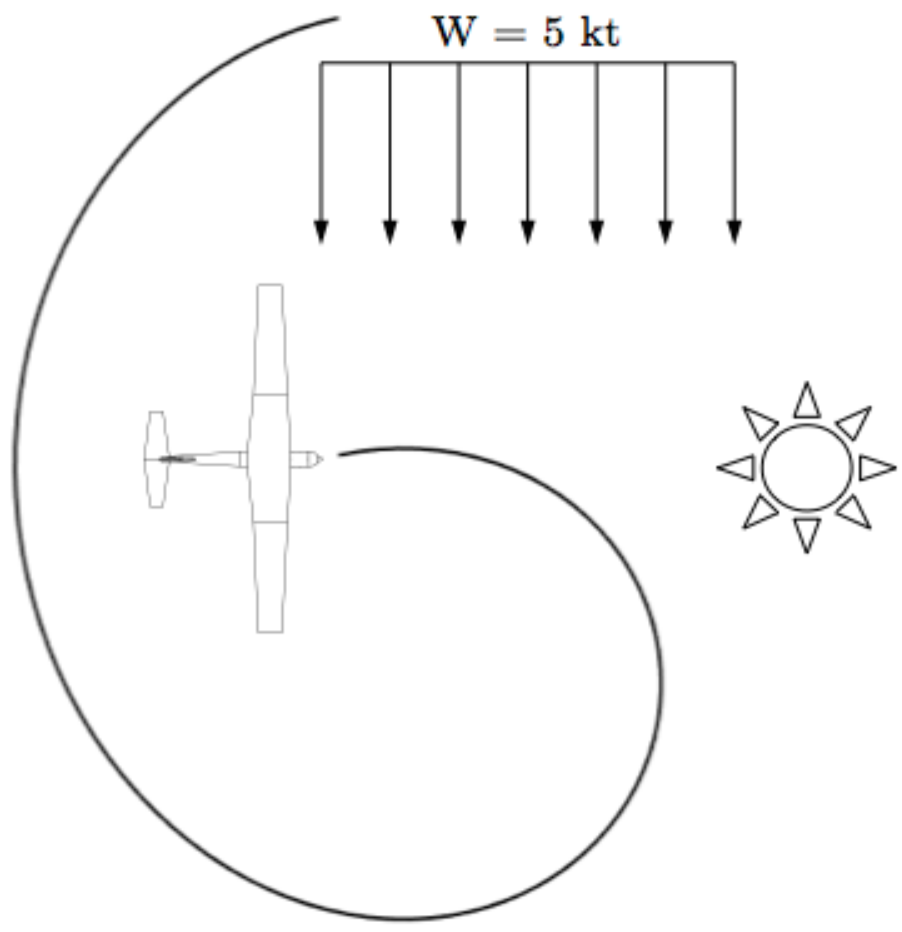

Figure 6.6: Example of path aircraft will fly through the air in order to maintain a circular ground track in the presence of winds as shown.

was varied to all four cardinal directions for the same desired circular orbit radius, wind speed of $5 \mathrm{kt}$, and sun position as described above. For these results shown in Table 6.2, wind direction is specified as the heading that the wind is out of; i.e., wind direction of 090 degrees indicates that the wind is coming from the east towards the west.

Table 6.2: Comparison of net energy for a circular orbit of radius $400 \mathrm{ft}$, with varying wind directions at $5 \mathrm{kt}$.

\begin{tabular}{|l|c|}
\hline Wind Direction (deg) & Net Energy \\
\hline 360 & $1289 \mathrm{~J}$ \\
090 & $1078 \mathrm{~J}$ \\
180 & $1071 \mathrm{~J}$ \\
270 & $1278 \mathrm{~J}$ \\
\hline
\end{tabular}

While Table 6.2 confirms these results, it also shows that a wind out of the west 
results in efficiency nearly as good as if the wind were out of the north. For best efficiency then, the wind should be oriented to result in an orbit track where the long upwind leg is "concave towards" the sun and the shorter downwind leg is concave away from the sun, as in Fig. 6.6. In the real world, one of course cannot choose the direction of the wind and sun, and for circular ground track orbits, there is no real input that can be changed freely besides orbit radius - on which the conclusions of the previous section should stand irrespective of winds.

\subsection{Elliptical Orbit Performance}

A simple variation from a circle is the ellipse, the shape of which is described with a major and minor axis with the minor being the smaller axis. The conclusions of the circular orbit radius study in Sec. 6.1 are unlikely to change by flying an elliptical pattern, where turn radius will have the same effects on aircraft efficiency but simply vary throughout the pattern. Thus, the orientation of the elliptical orbit in terms of major axis offset is of interest in this section, which is illustrated in Fig, 6.7.

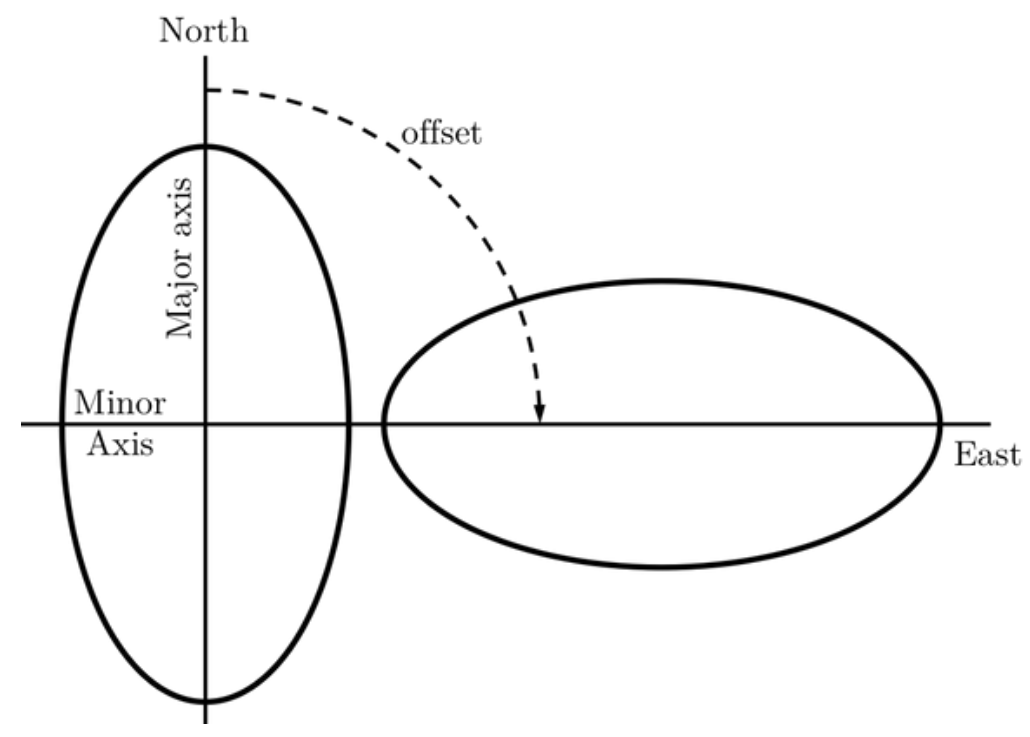

Figure 6.7: Illustration of terminology used to describe elliptical orbits. 
In order to investigate the effect of ellipse offset from north on aircraft efficiency, offset was swept through 180 degrees for various times of day (corresponding with elevation angles) on the summer and winter solstices and summer equinox. Semi-major axis was set to $400 \mathrm{ft}$, and semi-minor axis $200 \mathrm{ft}$. This is an ellipse comparable in size to the circles shown in Sec. 6.1 and is used in all results in this section unless otherwise noted. This situation is somewhat similar to the study of winds on circular orbits, and in this case since the shape is not symmetric about any axis through its center, offset from north up to 180 degrees will matter. In order to make a comparison between orbit offsets, azimuth angle is set to 90 degrees (sun in the east), while elevation angle varies with time according to its motion on the summer solstice. Results are shown in Fig.

\section{8 .}

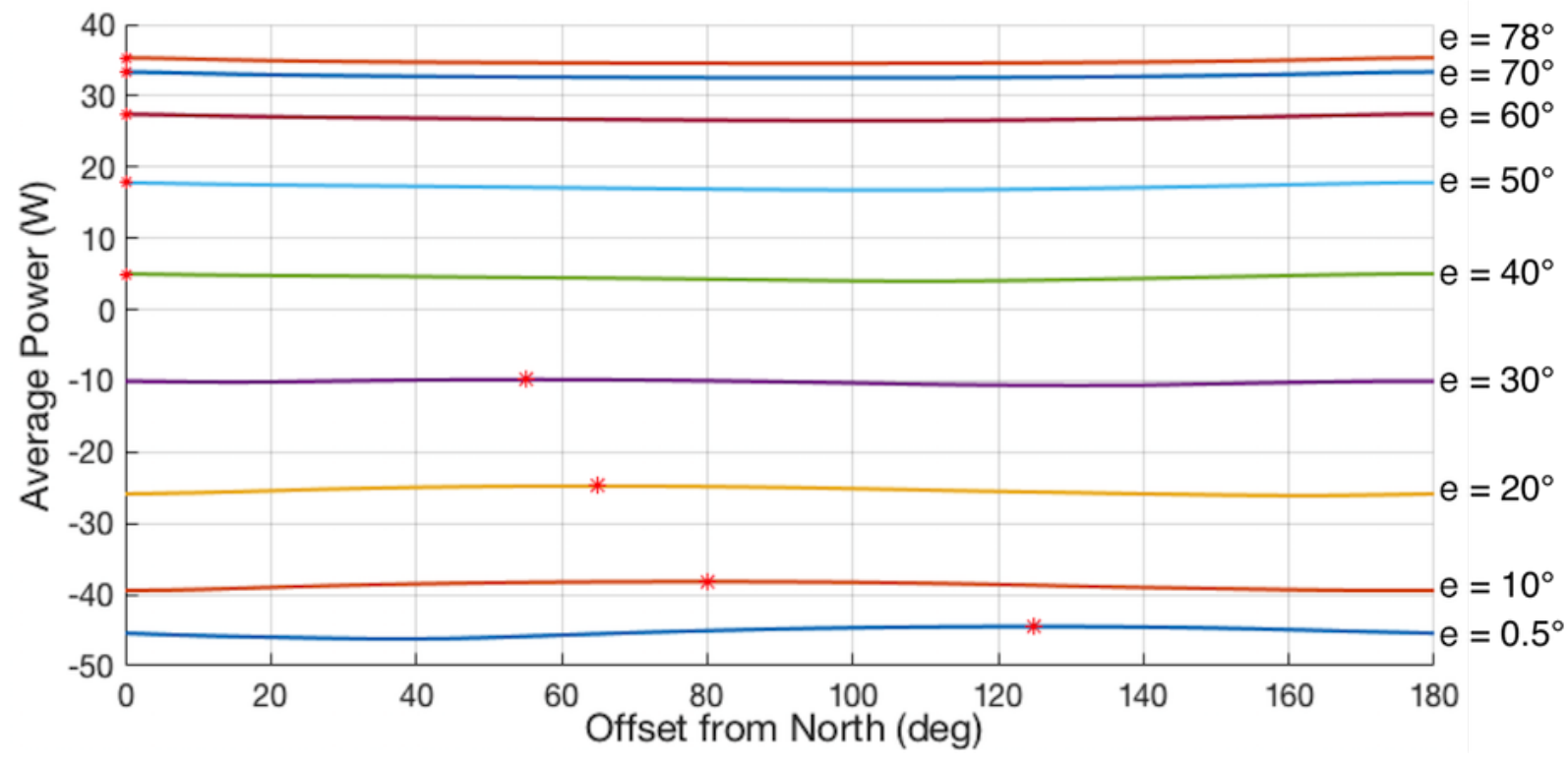

Figure 6.8: Average Power for asymmetric flight with varying orbit offset throughout the day on the summer solstice in San Luis Obispo, CA. Azimuth angle is set to 90 degrees for all cases.

For sun elevation angles below about 40 degrees, there is some efficiency gained by changing orbit offset. Past that angle, the best offset for net efficiency is zero degrees, which is likely because the sun is placed in the east, or an azimuth angle of 90 degrees. 
Best efficiency is most likely found for these higher elevation angles where the offset angle of the major axis is 90 degrees from the sun azimuth angle.

Insight can also be gained into the efficacy of asymmetric flight over conventional flight for elliptical orbits. As before, Table 6.3 shows a comparison of net energy between conventional and asymmetric flight for different times of day (elevation angles) and orbit offsets, with the sun placed in the east for all cases. Asymmetric flight shows improvement over conventional flight in all cases, to varying degrees. The large percent improvements for the 40 degree elevation case are previously unseen and it is unknown as to why there is such a large improvement for that specific case.

Table 6.3: Comparison of Net Energy for different orbit offsets throughout the summer solstice in San Luis Obispo, CA with the sun placed in the east.

\begin{tabular}{|c|c|c|c|c|}
\hline $\begin{array}{c}\text { Elevation } \\
\text { Angle }\end{array}$ & $\begin{array}{c}\text { Orbit } \\
\text { Offset }\end{array}$ & $\begin{array}{c}\text { Conventional } \\
\text { Flight } \\
\text { Net Energy }\end{array}$ & $\begin{array}{c}\text { Asymmetric } \\
\text { Flight } \\
\text { Net Energy }\end{array}$ & $\begin{array}{c}\% \\
\text { Improvement }\end{array}$ \\
\hline $10^{\circ}$ & $0^{\circ}$ & $-965 \mathrm{~J}$ & $-941 \mathrm{~J}$ & $2.5 \%$ \\
& $45^{\circ}$ & $-942 \mathrm{~J}$ & $-918 \mathrm{~J}$ & $2.6 \%$ \\
& $90^{\circ}$ & $-926 \mathrm{~J}$ & $-912 \mathrm{~J}$ & $1.5 \%$ \\
& $135^{\circ}$ & $-943 \mathrm{~J}$ & $-930 \mathrm{~J}$ & $1.4 \%$ \\
\hline $40^{\circ}$ & $0^{\circ}$ & $77.9 \mathrm{~J}$ & $120 \mathrm{~J}$ & $42.5 \%$ \\
& $45^{\circ}$ & $70.8 \mathrm{~J}$ & $109 \mathrm{~J}$ & $42.5 \%$ \\
& $90^{\circ}$ & $75.7 \mathrm{~J}$ & $99.0 \mathrm{~J}$ & $26.7 \%$ \\
& $135^{\circ}$ & $74.3 \mathrm{~J}$ & $102 \mathrm{~J}$ & $31.4 \%$ \\
\hline $70^{\circ}$ & $0^{\circ}$ & $786 \mathrm{~J}$ & $796 \mathrm{~J}$ & $1.3 \%$ \\
& $45^{\circ}$ & $770 \mathrm{~J}$ & $781 \mathrm{~J}$ & $1.4 \%$ \\
& $90^{\circ}$ & $770 \mathrm{~J}$ & $777 \mathrm{~J}$ & $0.9 \%$ \\
& $135^{\circ}$ & $772 \mathrm{~J}$ & $781 \mathrm{~J}$ & $1.2 \%$ \\
\hline
\end{tabular}

One more case of interest is the winter solstice. Results in Fig. 6.9 also suggest that for low sun elevation angles, there is some orbit offset for best average power. From the data, it appears that there is a break-point elevation angle around 30 degrees where the major axis of the ellipse should start pointing more and more towards the sun (in the east here) for best efficiency. 


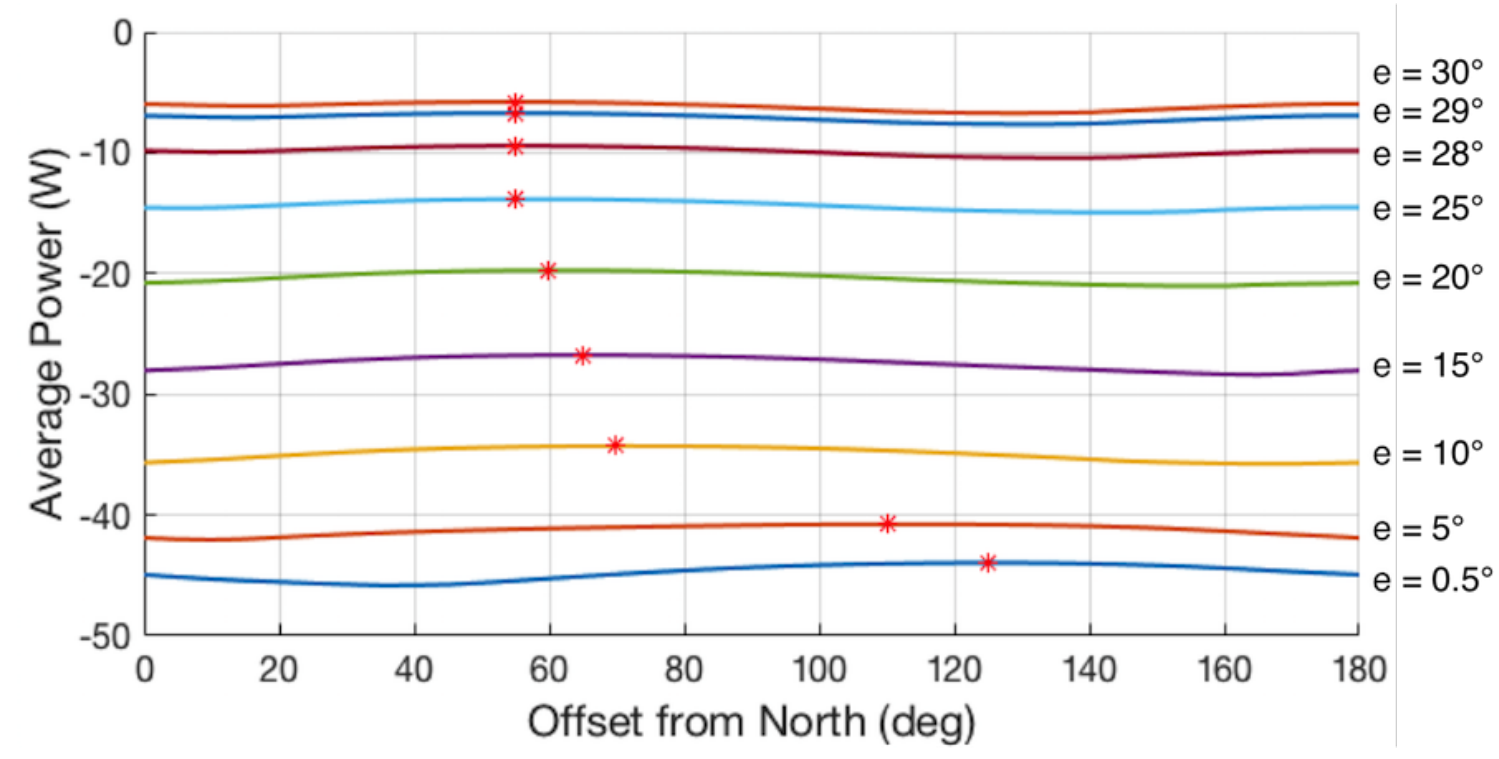

Figure 6.9: Average Power for asymmetric flight with varying orbit offset throughout the day on the winter solstice in San Luis Obispo, CA. Azimuth angle is set to 90 degrees for all cases.

\subsubsection{Winds on Elliptical Orbits}

Winds aloft will affect aircraft flight in elliptical orbits in much the same way as in circular orbits: any leg flown downwind will be shorter than any leg flown upwind in order to fit an elliptical ground track. The flight path taken for an elliptical ground track in the presence of winds from the north is shown in Fig. 6.10.

As concluded previously, it is likely that for better efficiency the longer, upwind leg of the orbit should be concave towards the sun. In the case of Fig. 6.10 the wind/sun interaction is detrimental and can only be reversed via placing the sun in the west or the wind out of the north. In the case of circular orbits, there is nothing that can be done; however, with elliptical orbits, one can adjust the offset angle of the major axis as investigated previously. To investigate this, various orbit offsets were run for the same sun position in the east, elevation angle of 45 degrees, and wind out of the north, and results are shown in Table 6.4. 


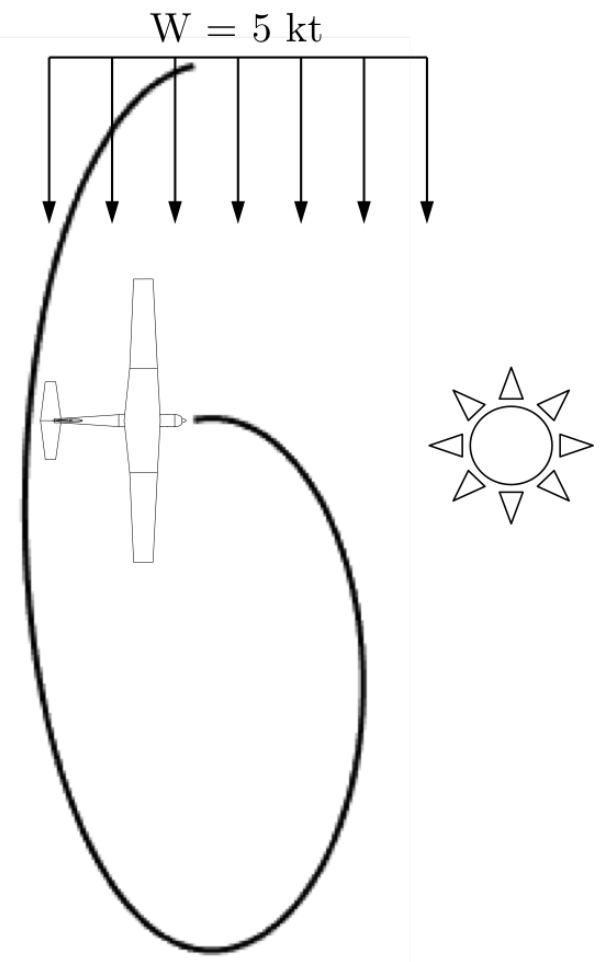

Figure 6.10: Example of path aircraft will fly through the air in order to maintain an elliptical ground track in the presence of winds as shown.

As expected, the best net energy case is the 0 degree orbit offset case. With this combination of factors (winds out of the north, sun in the east, left-turning orbit), the longer upwind leg is concave towards the sun. The model results show, though, that changing orbit offset from the case shown in Fig. 6.10 doesn't actually help net energy, and in this case the best orbit is to place the short downwind leg concave towards the sun.

Table 6.4: Comparison of net energy for an elliptical orbit, varying orbit offset, with wind at $360^{\circ}, 5 \mathrm{kt}$ and the sun in the east at elevation 45 degrees.

\begin{tabular}{|l|c|}
\hline Orbit Offset (deg) & Net Energy \\
\hline 0 & $412 \mathrm{~J}$ \\
045 & $396 \mathrm{~J}$ \\
090 & $380 \mathrm{~J}$ \\
135 & $390 \mathrm{~J}$ \\
\hline
\end{tabular}


With the wind now out of the south and the sun still in the east, lower net energies should be seen since winds now result in the long leg of the orbit being oriented concave away from the sun, as shown in Table 6.5. Since there is now a long period of time where the aircraft's bank angle is biased away from the sun in that long upwind leg, net energy shows reduction. Best case is still for an offset of 0 degrees, meaning that in this case it is best to place the shorter leg concave towards the sun. Alternatively, one could change from a right-turning to left-turning orbit and obtain the higher net energies of Table 6.4.

The data here supports the conclusion of Sec. 6.1 that larger orbit radii show better efficiency than smaller, since better net energies are seen here when the larger side of the ellipse is placed concave towards the sun than when the smaller side is concave towards the sun. It appears that even though smaller orbit radii will typically result in better incidence angles, the improvement in solar energy gained is outweighed by the increase in induced and trim drag associated with flight at higher load factors.

\section{Table 6.5: Comparison of net energy for an elliptical orbit, varying orbit offset, with wind at $180^{\circ}, 5 \mathrm{kt}$ and the sun in the east at elevation 45 degrees.

\begin{tabular}{|l|c|}
\hline Orbit Offset (deg) & Net Energy \\
\hline 0 & $222 \mathrm{~J}$ \\
045 & $205 \mathrm{~J}$ \\
090 & $187 \mathrm{~J}$ \\
135 & $197 \mathrm{~J}$ \\
\hline
\end{tabular}

With the wind out of the north or south and the sun in the east, then, it is best to place the major axis of the ellipse parallel with the wind. To see if this conclusion holds for a wind/sun orientation not offset by 90 degrees, wind direction was set to 045 degrees with the sun still in the east. As shown in Table 6.6, the best orientation for the ellipse is still with the major axis pointing north, offset 90 degrees from the sun. While there are multiple other factors that can affect this result, a potential strategy for 
best elliptical orbit is to place the major axis of the orbit offset from the azimuth angle of the sun by 90 degrees.

Table 6.6: Comparison of net energy for an elliptical orbit, varying orbit offset, with wind at $045^{\circ}, 5 \mathrm{kt}$ and the sun in the east at elevation 45 degrees.

\begin{tabular}{|l|c|}
\hline Orbit Offset (deg) & Net Energy \\
\hline 0 & $354 \mathrm{~J}$ \\
045 & $344 \mathrm{~J}$ \\
090 & $328 \mathrm{~J}$ \\
135 & $330 \mathrm{~J}$ \\
\hline
\end{tabular}

\subsection{Grid Flight Performance}

One final area of particular interest for small UAS is land survey. Typically, this means flying an aircraft over an area of interest with some kind of sensor mounted on the aircraft looking downward. Grid patterns are most conventionally used for coverage of a given area, and is plausible that grids could be constructed in such a way to best take advantage of both asymmetric flight and solar power in general.

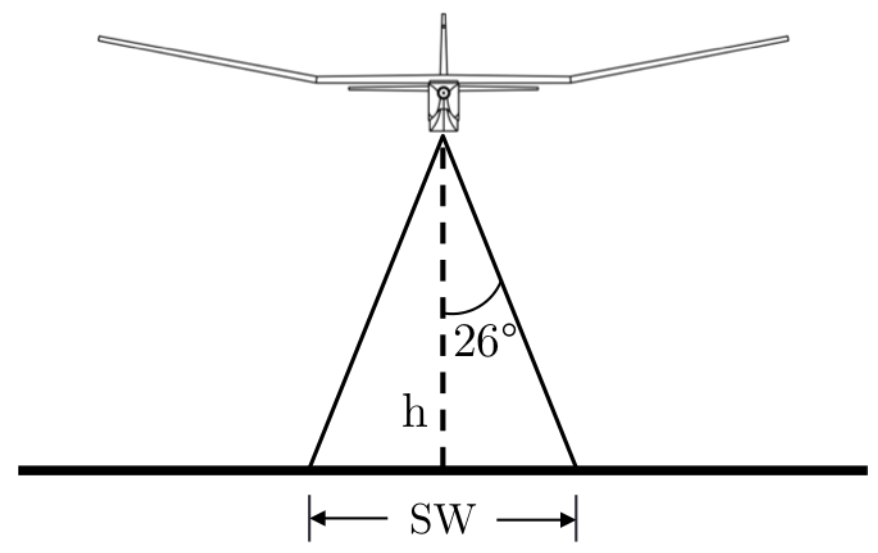

Figure 6.11: Illustration of the calculation of sensor swath width based on field of view angle.

Grid spacing should be set by swath width of the selected sensor and desired overlap between swaths. Specifics about the sensor must be known to calculate the swath 
width; one real example of a UAS-mounted hyperspectral sensor is the SHARK manufactured by NovaSol (now Corning). For the SHARK's field of view of 52 degrees [18], swath width is calculated from Fig. 6.11 with an input altitude $h$. For the $200 \mathrm{ft}$ MSL altitude used throughout this study, the corresponding swath width is $195.1 \mathrm{ft}$.

A grid can be approximated by 2 flight segments going in opposite directions with circular turns linking each end. If a uniform, rectangular grid is used to scan an entire area of interest, efficiency results for the first 2 segments can be applied via symmetry of aircraft orientation to the rest of the grid pattern. This approach assumes away factors like movement of the sun in the sky, but for a small UAS and a small area of interest, results are still applicable. An illustration of the ground track used to approximate grid flight is shown in Fig. 6.12.

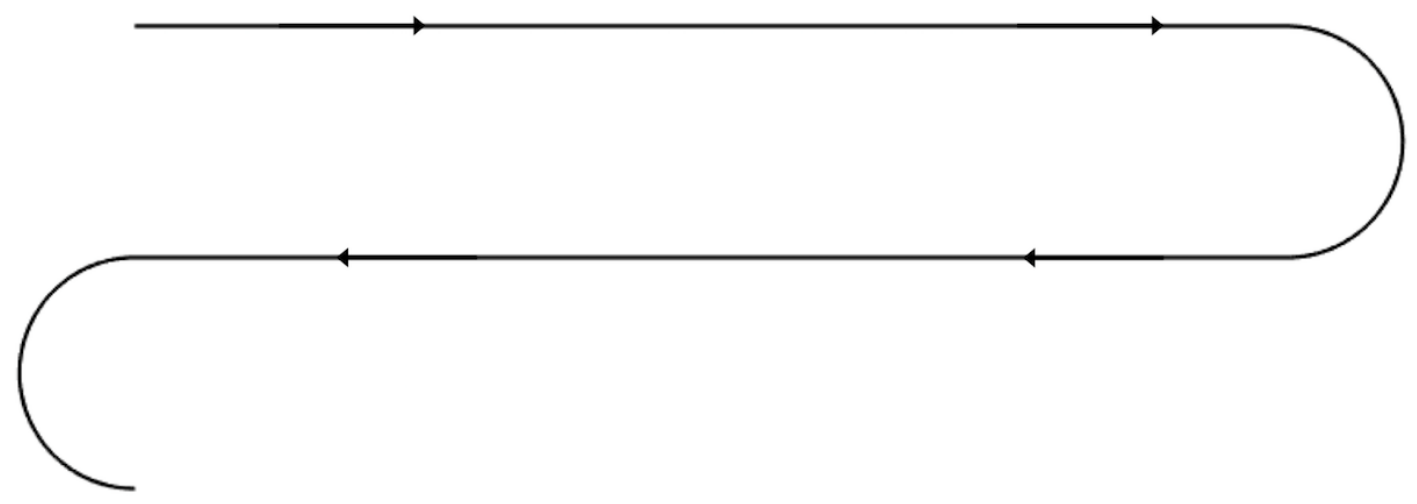

Figure 6.12: The first two segments of a land survey grid, used to assess grid mission performance.

Grid lines are placed such that they are one full swath width apart, and the mission is modeled such that the aircraft will always turn right first, as shown. Length of the grid lines (500 ft), swath width, aircraft altitude, and solar azimuth angle will be held constant; elevation angle and grid offset will be varied as before. Grid offset is defined similarly to the orbit offset of Sec. 6.2, where the offset angle is defined as the angular distance between due north and the heading of the first grid leg. As shown in Fig. 6.12, 
the grid offset is 90 degrees.

For the summer solstice, the results in Fig. 6.13 show that best average power is found at a grid offset of 180 degrees from north. This is another result that is difficult to intuit without the model results to guide thinking. At first glance, one would expect the 0 and 180 degree cases to be symmetrical and therefore have identical average powers. However, because the aircraft flies at some positive angle of attack, it is more advantageous to fly turns where the aircraft turns away from the sun, as that angle of attack then benefits solar incidence angle. This is the same reason why grid offsets of 90 degrees show worse performance than 180 degrees, since during one of the straight segments where the aircraft is flying directly towards the sun, that positive angle of attack adversely affects incidence angle. By the same token, on the return leg of the opposite heading, angle of attack helps incidence angle, but the results show that flying perpendicular to the sun for both legs is more efficient.

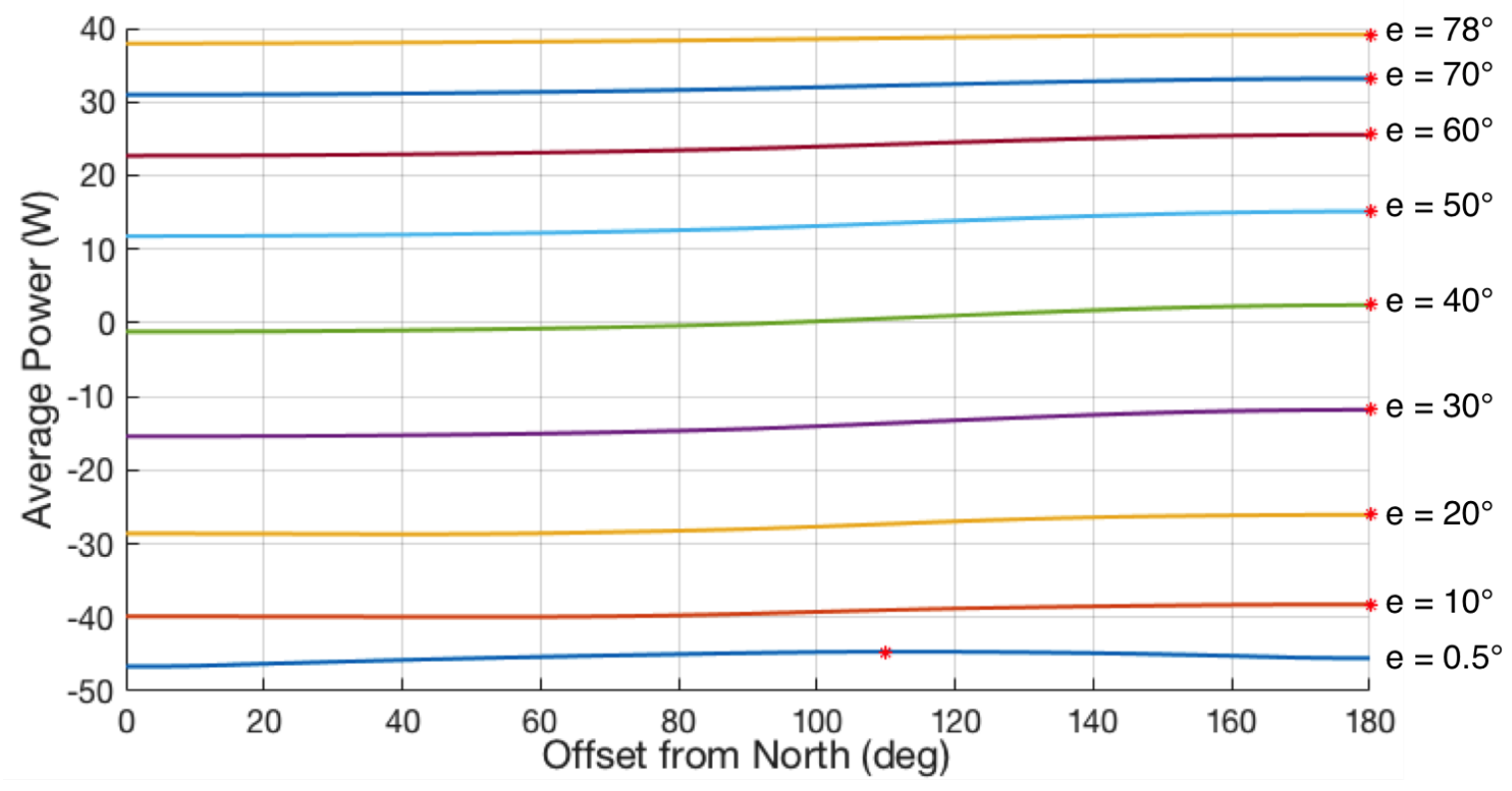

Figure 6.13: Average Power for asymmetric flight with varying grid offset throughout the day on the summer solstice in San Luis Obispo, CA. Azimuth angle is set to 90 degrees for all cases. 
In the winter, all average powers decrease as usual, and it is again shown that best average power is found at a grid offset of 180 degrees for practically all sun elevation angles. This indicates that the most efficient grid pattern, if the sun is in the east, is one where the straight segments are offset by 90 degrees from the sun's azimuth angle, and where the aircraft is turning away from, not towards, the sun in the turn segments.

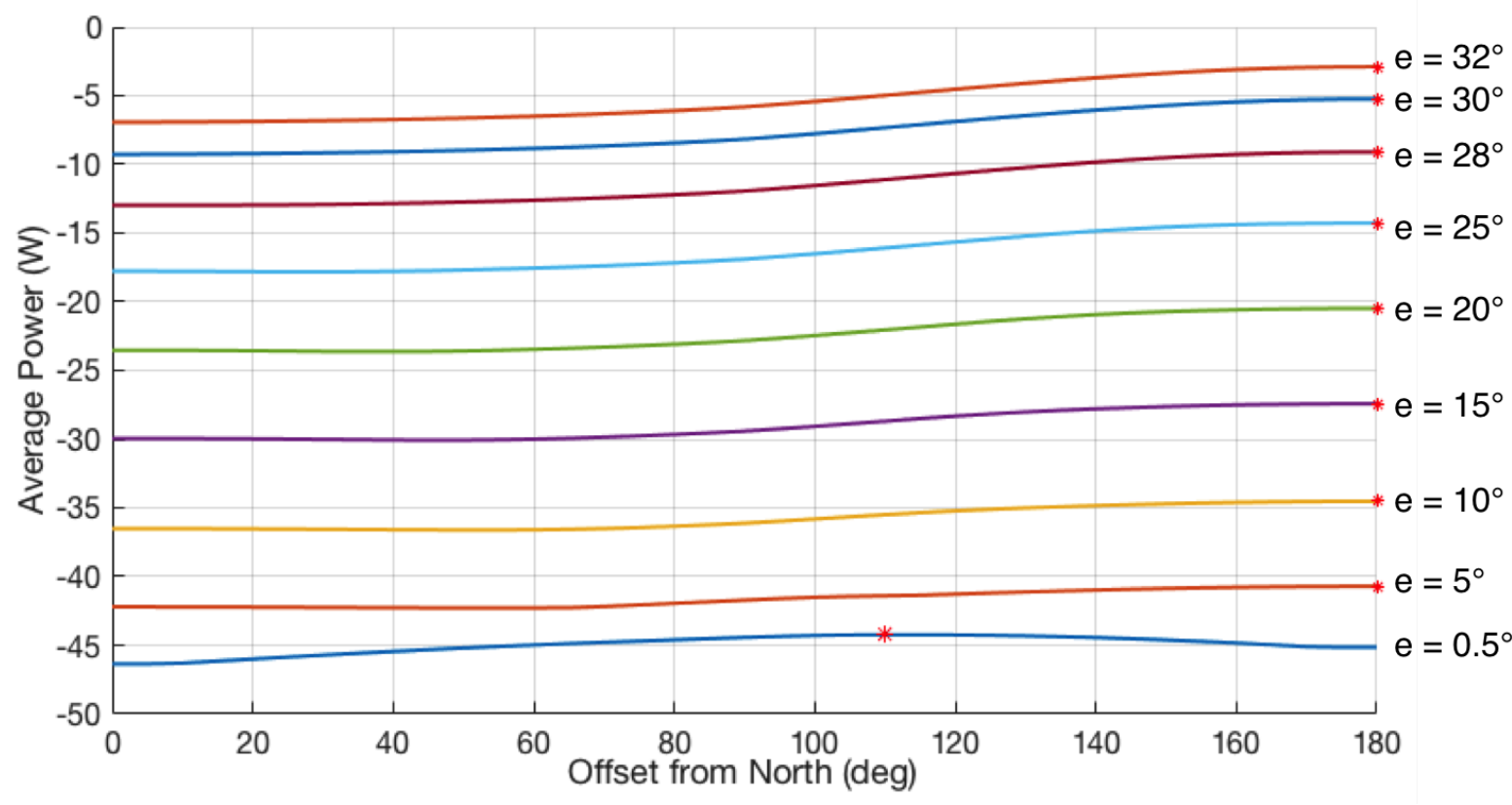

Figure 6.14: Average Power for asymmetric flight with varying grid offset throughout the day on the winter solstice in San Luis Obispo, CA. Azimuth angle is set to 90 degrees for all cases.

The relative improvement between asymmetric flight and conventional flight should also be considered, as shown in Table 6.7. Asymmetric flight again shows some efficiency improvement for all cases. In general, asymmetric flight shows less improvement for grid flights than circle or elliptical orbits. Again there are some unexpectedly large improvements at 40 degrees elevation, perhaps showing that there is some kind of sweet spot for asymmetric flight in that elevation range.

Overall, results seem to show that it is best to place grid offset 90 degrees from the sun's azimuth angle, and set so that the aircraft turns away from the sun. Turns exe- 
Table 6.7: Comparison of Net Energy for different grid offsets throughout the summer solstice in San Luis Obispo, CA with the sun placed in the east.

\begin{tabular}{|c|c|c|c|c|}
\hline $\begin{array}{c}\text { Elevation } \\
\text { Angle }\end{array}$ & $\begin{array}{c}\text { Grid } \\
\text { Offset }\end{array}$ & $\begin{array}{c}\text { Conventional } \\
\text { Flight } \\
\text { Net Energy }\end{array}$ & $\begin{array}{c}\text { Asymmetric } \\
\text { Flight } \\
\text { Net Energy }\end{array}$ & $\begin{array}{c}\text { \% } \\
\text { Improvement }\end{array}$ \\
\hline $10^{\circ}$ & $0^{\circ}$ & $-1618 \mathrm{~J}$ & $-1581 \mathrm{~J}$ & $2.3 \%$ \\
& $45^{\circ}$ & $-1610 \mathrm{~J}$ & $-1584 \mathrm{~J}$ & $1.75 \%$ \\
& $90^{\circ}$ & $-1582 \mathrm{~J}$ & $-1568 \mathrm{~J}$ & $0.89 \%$ \\
& $135^{\circ}$ & $-1557 \mathrm{~J}$ & $-1530 \mathrm{~J}$ & $1.75 \%$ \\
& $180^{\circ}$ & $-1550 \mathrm{~J}$ & $-1517 \mathrm{~J}$ & $2.15 \%$ \\
\hline $40^{\circ}$ & $0^{\circ}$ & $-107 \mathrm{~J}$ & $-48 \mathrm{~J}$ & $55.1 \%$ \\
& $45^{\circ}$ & $-87 \mathrm{~J}$ & $-39 \mathrm{~J}$ & $55.2 \%$ \\
& $90^{\circ}$ & $-39 \mathrm{~J}$ & $-6 \mathrm{~J}$ & $84.6 \%$ \\
& $135^{\circ}$ & $9 \mathrm{~J}$ & $61 \mathrm{~J}$ & $578 \%$ \\
& $180^{\circ}$ & $28 \mathrm{~J}$ & $96 \mathrm{~J}$ & $110 \%$ \\
\hline $70^{\circ}$ & $0^{\circ}$ & $1423 \mathrm{~J}$ & $1434 \mathrm{~J}$ & $0.77 \%$ \\
& $45^{\circ}$ & $1431 \mathrm{~J}$ & $1440 \mathrm{~J}$ & $0.63 \%$ \\
& $90^{\circ}$ & $1449 \mathrm{~J}$ & $1458 \mathrm{~J}$ & $0.62 \%$ \\
& $135^{\circ}$ & $1468 \mathrm{~J}$ & $1483 \mathrm{~J}$ & $1.02 \%$ \\
& $180^{\circ}$ & $1475 \mathrm{~J}$ & $1495 \mathrm{~J}$ & $1.35 \%$ \\
\hline
\end{tabular}

cuted in the right orientation will cause a positive angle of attack to improve incidence angle, which improves solar collection efficiency simply as a consequence of normal aircraft flight characteristics.

\subsubsection{Winds on Grid Flight}

Effects of winds on flight through rectangular grids will be handled as in the previous sections by setting sun position and varying wind direction and grid orientation. Since the grid pattern selected is a mix of both constant-heading and steady turning flight, it is harder to develop intuition or expectations on what results to expect. The first case shown is for varying grid offset from 0 to 315 degrees (essentially orienting the grid lines through all of the cardinal and semi-cardinal directions), with the sun as usual placed in the east at an elevation of 45 degrees, and winds of 5 knots out of 
the north. Because the aircraft carries an angle of attack, and a grid pattern involves both right and left turns, these grid patterns cannot be treated as symmetric through 180 degrees as with the elliptical and circular patterns before.

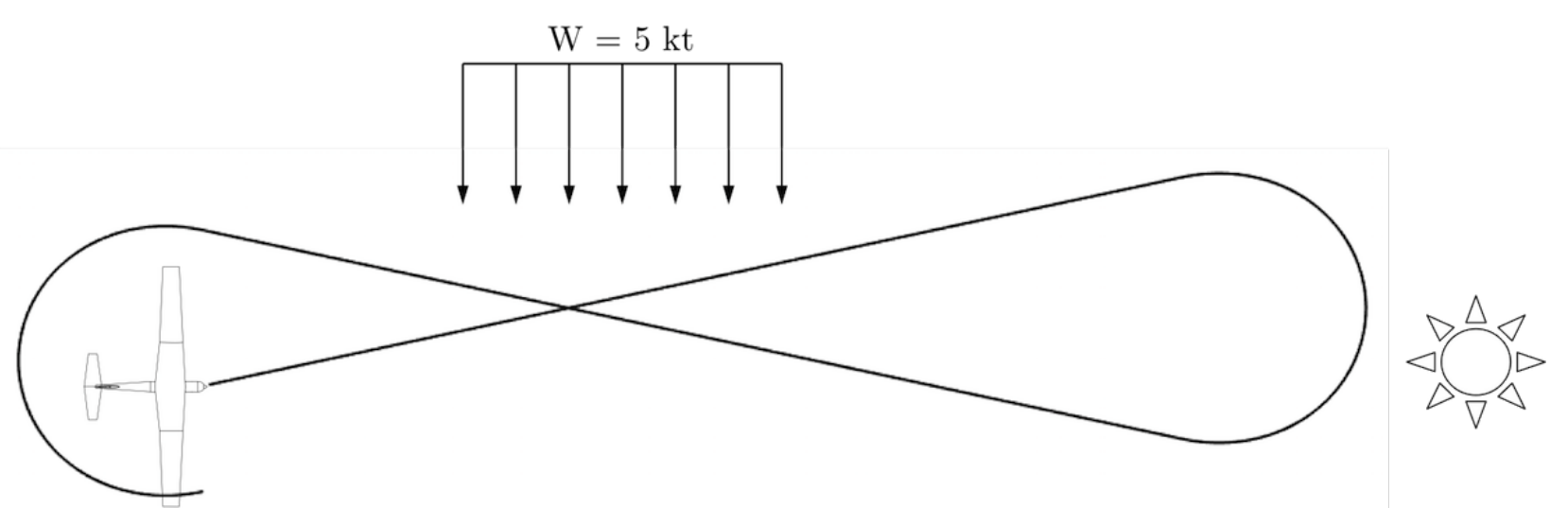

\section{Figure 6.15: Grid offset of $090^{\circ}$ under the effects of $5 \mathrm{kt}$ winds at $360^{\circ}$ with the sun in the east.}

The results in Table 6.8 show that, for the sun in the east and wind out of the north, the most efficient grid orientation is south-north (grid offset 180 degrees). Because the aircraft flies each straight segment (and turn segments) at some non-zero angle of attack, the straight segments in the north-south grid show better angles of incidence than those in segments with some easterly or westerly orientation, and therefore the north-south oriented grid shows better overall efficiency. Additionally, by comparing other grids offset from each other by 180 degrees, such as 045 vs. 225 degrees, it is shown that grids where the aircraft is flying and turning away from the sun show better efficiencies than those where the aircraft is turning towards the sun, again likely because of angle of attack effects.

With the wind out of the east, the situation is the same, as shown in Table 6.9. This indicates that the strong effect for grid flight is grid/sun orientation, not necessarily wind direction. In a grid where the majority of flight time is spent in straight lines, the effect of winds is mainly to change aircraft heading, not bank angle as in a circle 
Table 6.8: Comparison of net energy for a grid pattern, varying grid offset, with wind at $360^{\circ}, 5 \mathrm{kt}$ and the sun in the east at elevation 45 degrees.

\begin{tabular}{|l|c|}
\hline Grid Offset (deg) & Net Energy \\
\hline 0 & 551 J \\
045 & 548 J \\
090 & $575 \mathrm{~J}$ \\
135 & $649 \mathrm{~J}$ \\
180 & $698 \mathrm{~J}$ \\
225 & $665 \mathrm{~J}$ \\
270 & $595 \mathrm{~J}$ \\
315 & $562 \mathrm{~J}$ \\
\hline
\end{tabular}

or ellipse, and thus has a smaller effect on overall efficiency. While the 180 degree offset case is still the best, it shows less net energy with wind out of the east than the north. It is hard to draw general conclusions about a situation where wind can vary in any direction and strength, and the sun's position varies in two axes; but it appears that grid orientation and sun position are still the key trend-setting parameters, while winds serve to only change values within the trend.

Table 6.9: Comparison of net energy for a grid pattern, varying grid offset, with wind at $090^{\circ}, 5 \mathrm{kt}$ and the sun in the east at elevation 45 degrees.

\begin{tabular}{|l|c|}
\hline Grid Offset (deg) & Net Energy \\
\hline 0 & $491 \mathrm{~J}$ \\
045 & $500 \mathrm{~J}$ \\
090 & $529 \mathrm{~J}$ \\
135 & $581 \mathrm{~J}$ \\
180 & $606 \mathrm{~J}$ \\
225 & $580 \mathrm{~J}$ \\
270 & $527 \mathrm{~J}$ \\
315 & $499 \mathrm{~J}$ \\
\hline
\end{tabular}

With the sun in the west as in Table 6.10, the situation is reversed: best net energy is found for a grid offset of 0 degrees (north-south grid lines) instead of 180 degrees, and all other comparisons are swapped by 180 degrees as well. This adds credibility to the assertion that better grid patterns are found where the aircraft is turning away from 
the sun instead of towards the sun.

Table 6.10: Comparison of net energy for a grid pattern, varying grid offset, with wind at $090^{\circ}, 5 \mathrm{kt}$ and the sun in the west at elevation 45 degrees.

\begin{tabular}{|l|c|}
\hline Grid Offset (deg) & Net Energy \\
\hline 0 & $696 \mathrm{~J}$ \\
045 & $646 \mathrm{~J}$ \\
090 & $572 \mathrm{~J}$ \\
135 & $546 \mathrm{~J}$ \\
180 & $551 \mathrm{~J}$ \\
225 & $564 \mathrm{~J}$ \\
270 & $596 \mathrm{~J}$ \\
315 & $665 \mathrm{~J}$ \\
\hline
\end{tabular}

For grid flight, results shown in the effects of wind largely echo the same conclusions that can be made from the analysis performed without wind effects. It is best to fly a grid where the grid lines are offset by 90 degrees from the sun's azimuth angle, making turns away from the sun such that angle of attack benefits incidence angle. Winds will change magnitudes of the net energy for a particular grid, but are unlikely to change the grid offset for best net efficiency. 


\section{Chapter 7}

\section{CONCLUSION}

This thesis assesses the net benefit of using asymmetric flight to improve total solar aircraft efficiency. Asymmetric flight is an unconventional flight technique where an aircraft's bank angle is manipulated in order to point notionally wing-mounted solar cells towards the sun, while using sideslip to maintain a desired flight path despite that non-zero bank angle. At heart, this thesis is an analysis of the trade-off between increased solar energy from banking the aircraft towards the sun and increased drag as a result of flying in sideslip.

An aerodynamics model sensitive to sideslip angle and solar-photovoltaic model were built up and linked together in order to provide point performance predictions for an aircraft flying asymmetrically. That point performance model was then interrogated across a series of points defined such that an input mission was simulated. Efficiency improvements in terms of net power and net energy were found of up to $5 \%$ for an aircraft flying asymmetrically.

Effort was also made to consider the effects of winds aloft on mission performance, and the mission model has the capability to modify an aircraft's heading and ground velocity in order to maintain a desired ground track in the presence of winds of any input velocity and direction. One finding of note is that for best orbit efficiency, the upwind leg of an orbit should be flown concave towards the sun, if possible.

Ultimately, the efficiency gains and findings in this study are subject to multiple simplifying assumptions and aircraft-specific parameters, as detailed. With the small magnitude of efficiency gains found, it is possible that, for the conventional small UAS configuration used as a representative of its class, there is little to no efficiency to be 
gained after all. However, were effort to be expended to improve drag penalties associated with asymmetric flight, as well as improve solar collection efficiencies, there could be more value found with this type of flight. While the many pitfalls of this study ultimately limit its utility in making a conclusive decision about the benefits of asymmetric flight, it seems clear that there is value to be extracted from not only aircraft flight planning, but also orientation planning. 


\section{BIBLIOGRAPHY}

[1] Edwards, J. D., Kahn, A. D., and Kelly, M., et. al., "Maximizing Net Power in Circular Turns for Solar and Autonomous Soaring Aircraft," AIAA Journal of Aircraft, Article in Advance.

[2] Abbott, I. H., and Von Doenhoff, A. E., Theory of Wing Sections, Dover Publications, New York, 1959, pp. 454.

[3] Hoerner, S. F., Fluid-Dynamic Drag, Hoerner Fluid Dynamics, Vancouver, WA, 1965, pp. 3-11, 3-17.

[4] Tornado Vortex Lattice, Matlab code package, Ver. 135, T. Melin, Royal Institute of Technology, 2000.

[5] User's Guide and Reference Manual for Tornado, T. Melin, Royal Institute of Technology, 2000.

[6] Nicolai, L. M., Fundamentals of Aircraft Design, METS Inc., San Jose, CA, 1984, Chaps. 11, 12.

[7] Noth, A., "Design of Solar Powered Airplanes for Continuous Flight," Ph.D. Dissertation No. 18010, ETH Zrich, Zrich, Switzerland, 2008, doi:10.3929/ethza-005745622

[8] Duffie, J. A., and Beckman, W. A., Solar Engineering of Thermal Processes, $3^{\text {rd }}$ ed., John Wiley \& Sons, Hoboken, NJ, 2006, Chaps. 1, 2.

[9] Hottel, H. C., "A Simple Model for Estimating the Transmittance of Direct Solar Radiation Through Clear Atmospheres," Solar Energy, Vol. 18, No. 2, 1976, pp. 129-134. 
[10] Goldstein, H., Classical Mechanics, Addison-Wesley, London, 1959, pp. 107109.

[11] Glazebrook, G. M., and McDonald, R. A., "Solar Energy Collection Analysis Tool for Conceptual Aircraft Design," $51^{s t}$ AIAA Aerospace Sciences Meeting, AIAA, Washington, DC, 2013.

[12] Task Committee on Standardization of Reference Evapotranspiration, "The ASCE Standardized Reference Evapotranspiration Equation", American Society of Civil Engineers, 2005.

[13] STAR-CCM+, Software Package, Ver. 11.04.012, CD-adapco, Melville, NY, 2016.

[14] Matlab and Mapping Toolbox Release 2016A, Software Package, The MathWorks, Inc., Natick, Massachusetts.

[15] Anderson, J. D., Aircraft Performance and Design, McGraw-Hill, Boston, MA, 1999, Chap. 6.

[16] Apogee Instruments, 2016. SP-420 Owners Manual. Retrieved from http://www.apogeeinstruments.com/sp-420-smart-pyranometer-usb-output/.

[17] Clear-Sky Calculator. Apogee Instruments. Retrieved October 15, 2016, from http://clearskycalculator.com/pyranometer.htm

[18] Innovative Technical Solutions, Inc., 2015. SHARK Visible/Near Infra-Red Hyperspectral System Users Manual. Honolulu, HI.

[19] Sproul, A., "Derivation of the solar geometric relationships using vector analysis," Renewable Energy, Vol. 32, No. 7, 2007, pp. 1187-1205. 
[20] Klesh, A. T., and Kabamba, P. T., "Energy-Optimal Path Planning for SolarPowered Aircraft in Level Flight," AIAA Guidance, Navigation, and Control Conference and Exhibit, AIAA, Hilton Head, SC, 2007. 


\section{APPENDICES}

\section{Appendix A \\ TIME HISTORIES OF SELECTED MISSIONS}

In order to exercise the model and as a matter of interest, time histories of selected model outputs for each of the selected mission cases are shown: circular and elliptical orbits, and land survey grids. First, circular orbit time histories are shown where conditions are San Luis Obispo, CA summer solstice with the sun in the east and an elevation angle of 45 degrees. Since an orbit is treated in this study as a single sweep through 360 degrees of heading, it is convenient to show variation of a few parameters as a function of the aircraft's heading throughout its orbit. As shown in Fig. A.1, asymmetric bank angle changes for best net power throughout a circular orbit; in a conventional circular orbit, bank angle is constant.

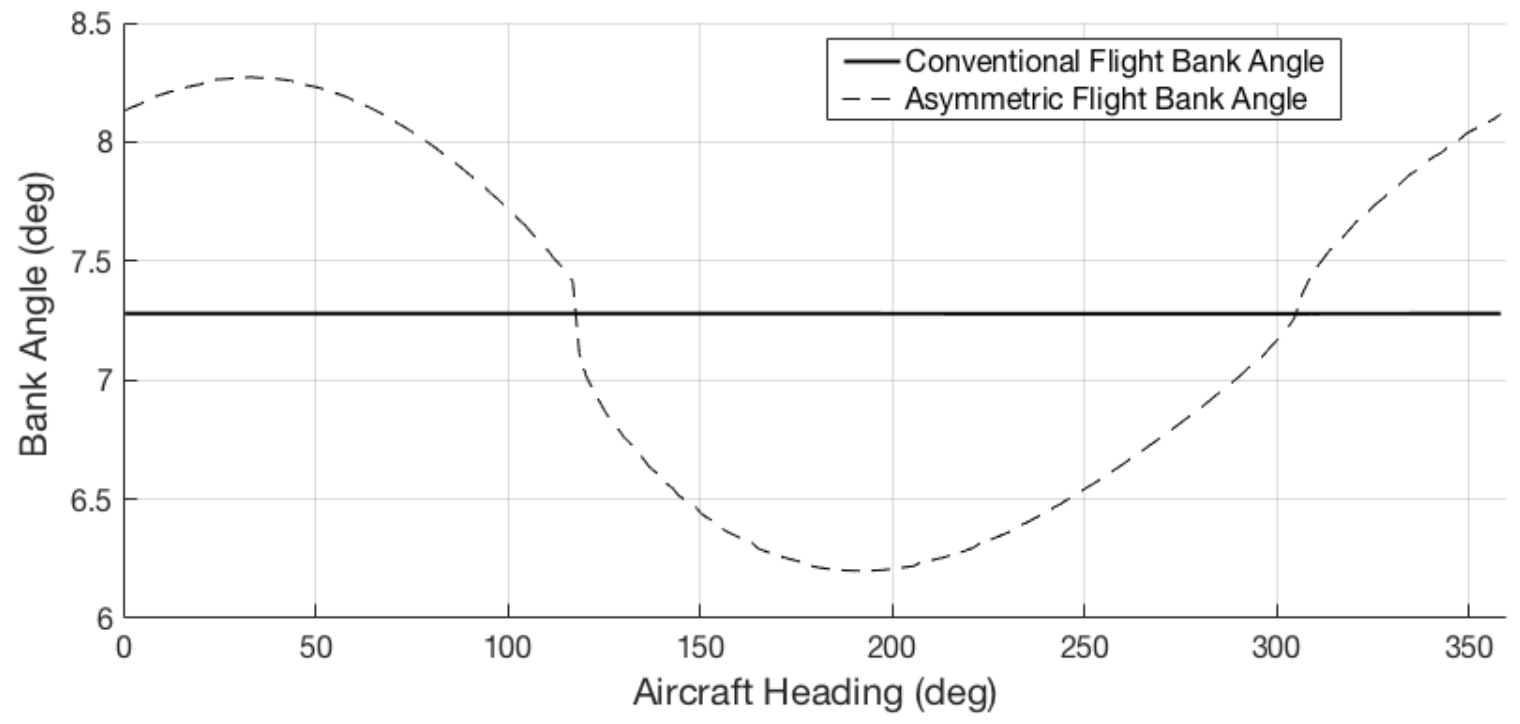

Figure A.1: Time history of bank angles for a sample circular orbit on the summer solstice in San Luis Obispo, CA. 
Similarly, a history of solar incidence angle during this selected circular orbit is shown in Fig. A.2. Of note is that asymmetric flight results in a lower solar incidence angle, to varying degrees, for every point in the orbit.

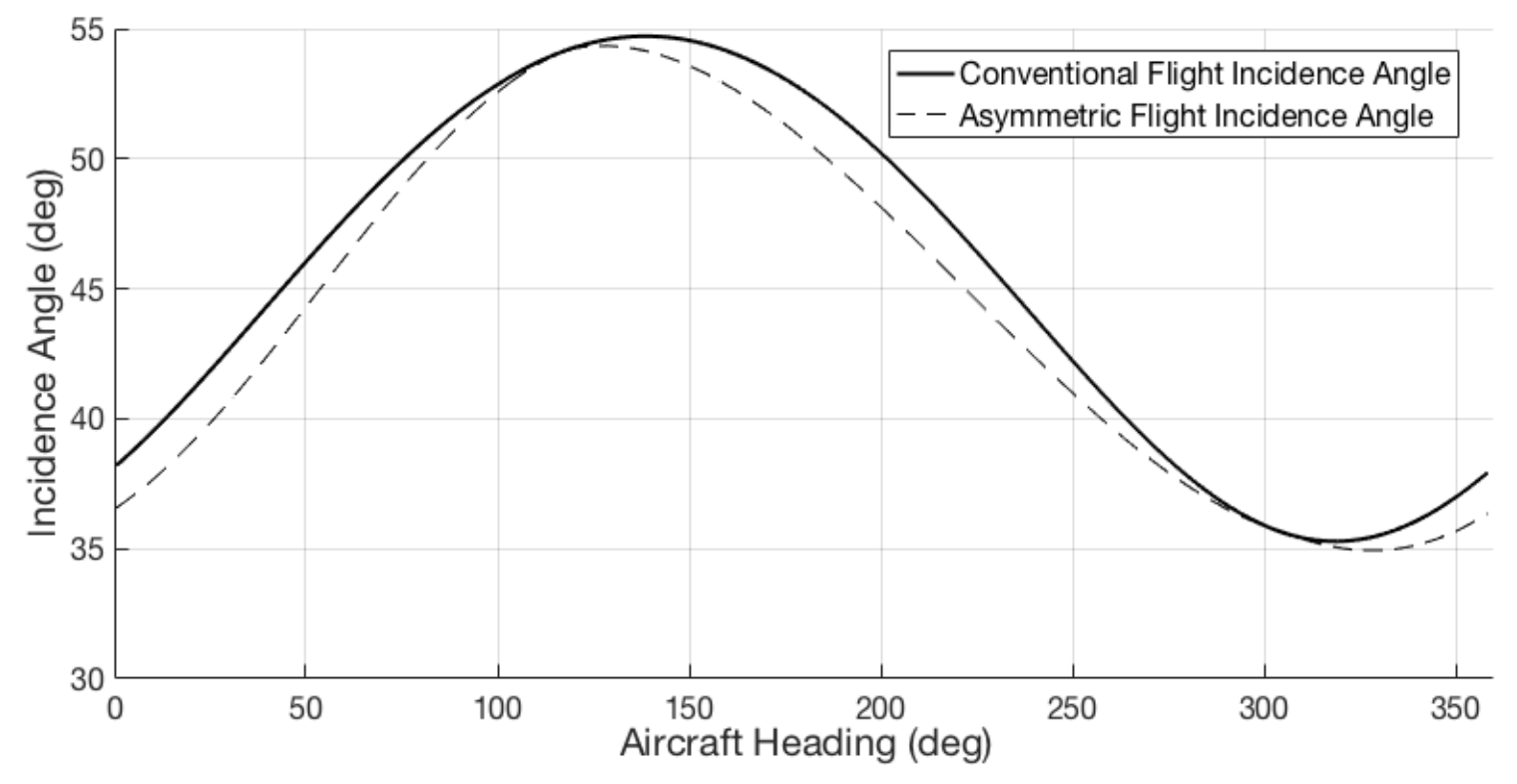

Figure A.2: Time history of solar incidence angles for a sample circular orbit on the summer solstice in San Luis Obispo, CA.

Finally, a history of net power is shown for both conventional and asymmetric flight on the same circular orbit in Fig. A.3. Once again, it is shown that asymmetric flight provides better net power throughout the orbit, varying with position along that orbit.

Some history plots for a sample elliptical orbit are also included and are for an ellipse offset of 0 degrees, with a major axis of $400 \mathrm{ft}$ and minor axis of $200 \mathrm{ft}$ with the same sun location as previous. It is shown in Fig. A.4 how asymmetric flight is used to bias the aircraft's bank angle towards the sun for best net power as compared with the bank angle required for coordinated conventional flight. It is interesting to note that in this specific case, the most asymmetric bank angle is seen around the maximum and minimum conventional bank angle values. 


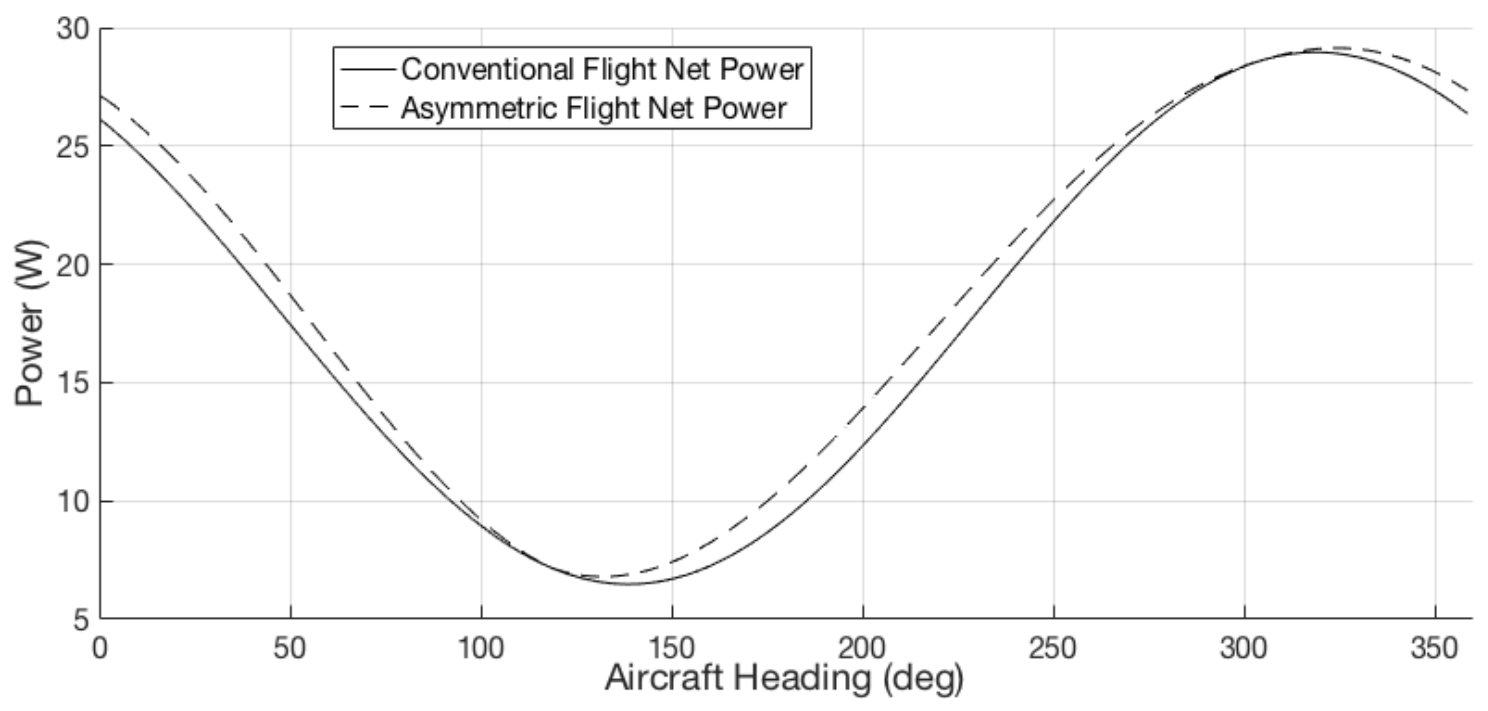

Figure A.3: Time history of net power for a sample circular orbit on the summer solstice in San Luis Obispo, CA.

A history of solar incidence angles throughout the same elliptical orbit in Fig. A.5 shows the resulting improvement in incidence angle due to manipulation of bank angle on a point-by-point basis throughout the mission. Since lower solar incidence angle is better for energy collection, improvement in incidence angle is seen almost across the board for asymmetric vs. conventional flight.

Finally, a time history of net power for both conventional and asymmetric flight is shown in Fig. A.6. Better improvements in net power are seen at different points throughout the orbit; this non-uniform improvement over conventional flight is to be expected from an elliptical orbit, where bank angle varies considerably due to its shape.

The same data is also shown for the land survey grids, again with the sun in the east at an elevation angle of 45 degrees on the summer solstice in San Luis Obispo, CA. The sample grid used is constructed identically to those used in this paper and the first leg is oriented to the north. The x-axis now shows elapsed time, as it is less practical to show aircraft heading in a mission where heading is not swept smoothly through 360 degrees. 


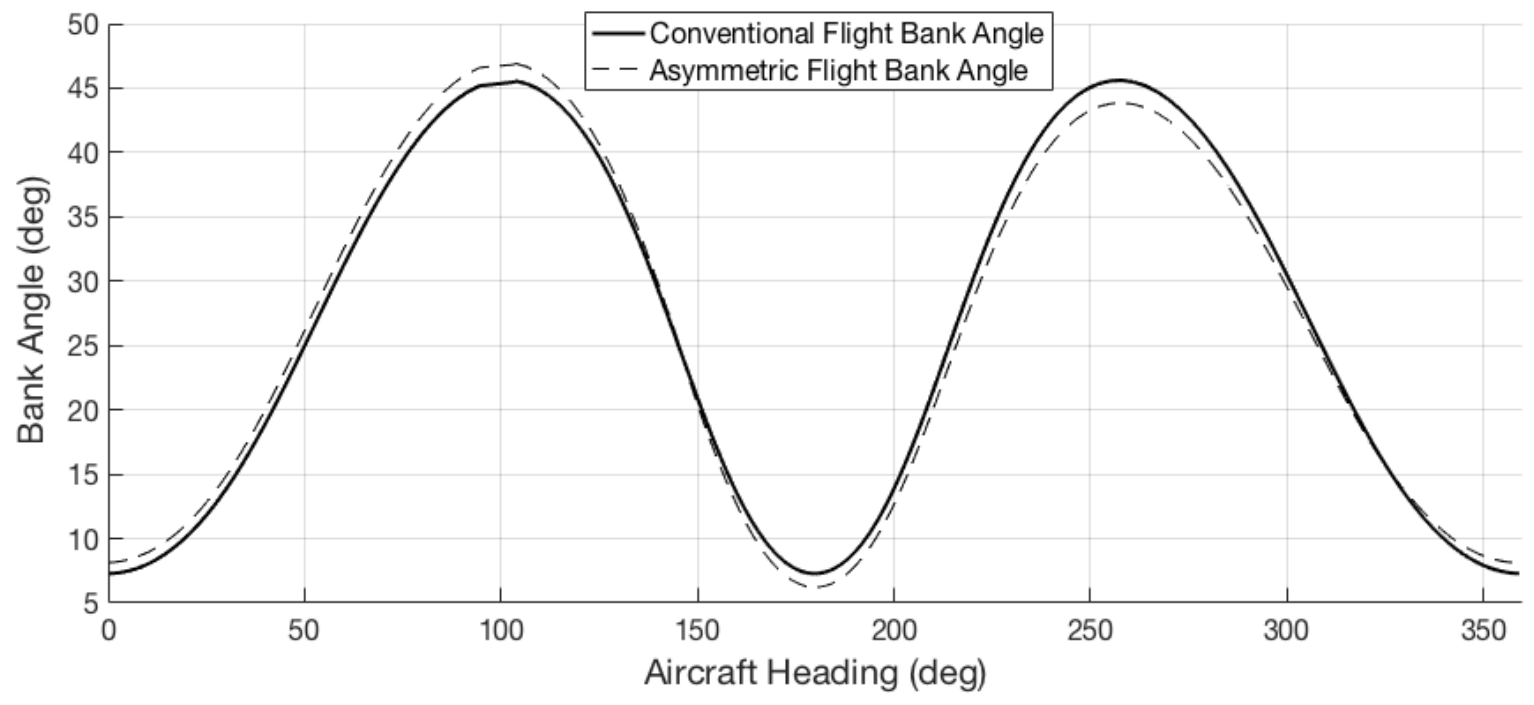

Figure A.4: Time history of bank angles for a sample elliptical orbit on the summer solstice in San Luis Obispo, CA.

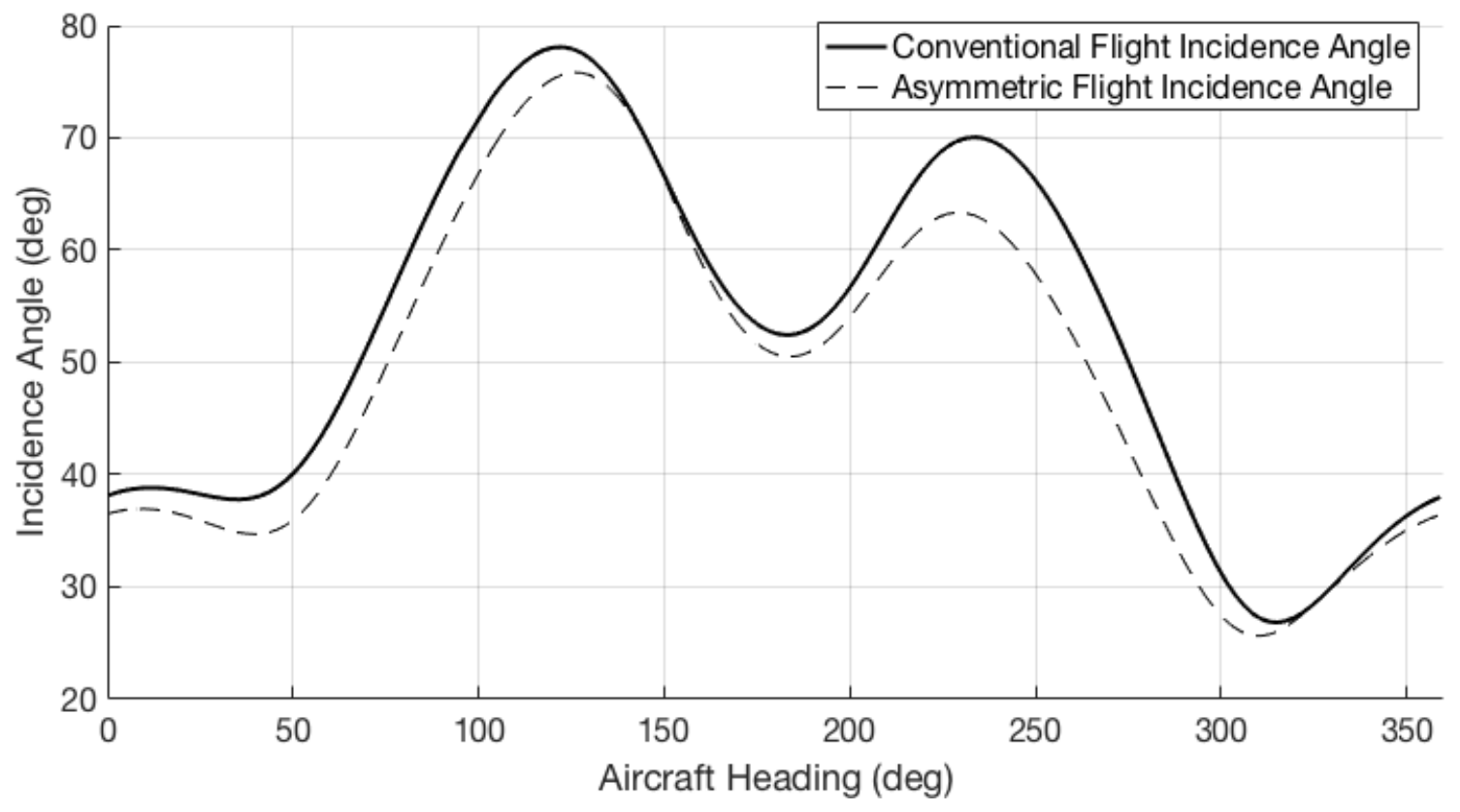

Figure A.5: Time history of solar incidence angles for a sample elliptical orbit on the summer solstice in San Luis Obispo, CA. 


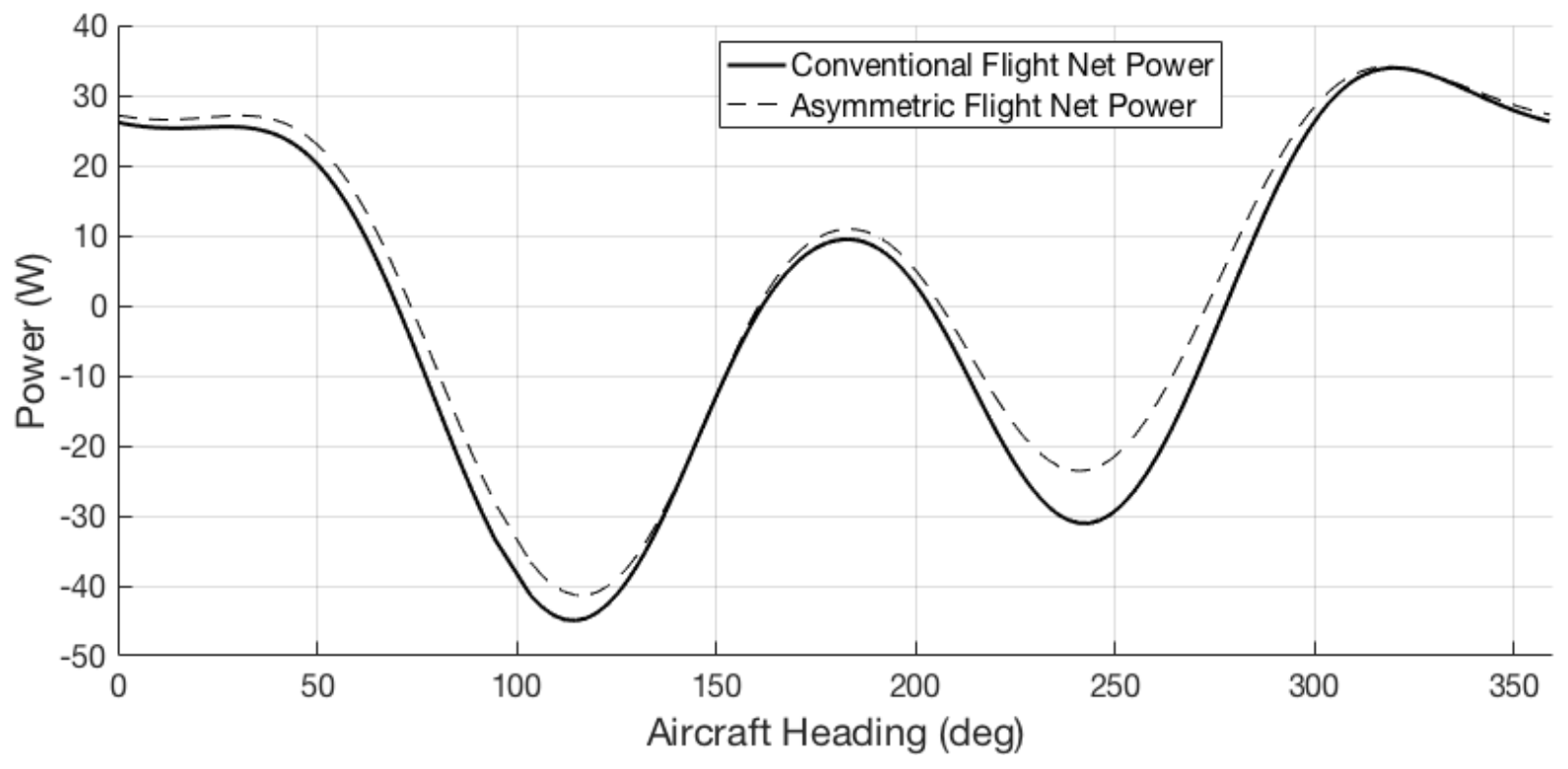

Figure A.6: Time history of net power for a sample elliptical orbit on the summer solstice in San Luis Obispo, CA.

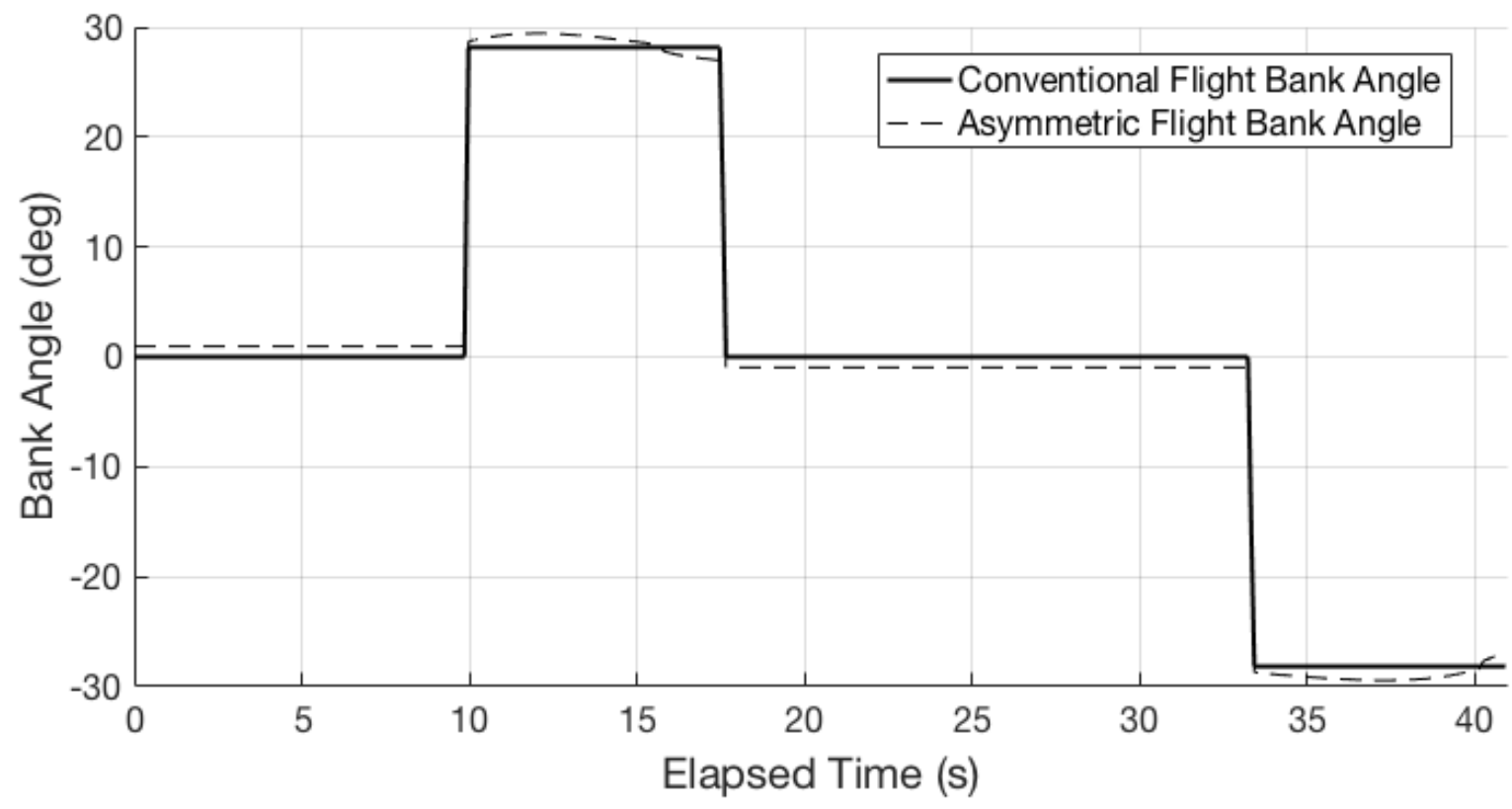

Figure A.7: Time history of bank angle for a sample grid flight on the summer solstice in San Luis Obispo, CA. 


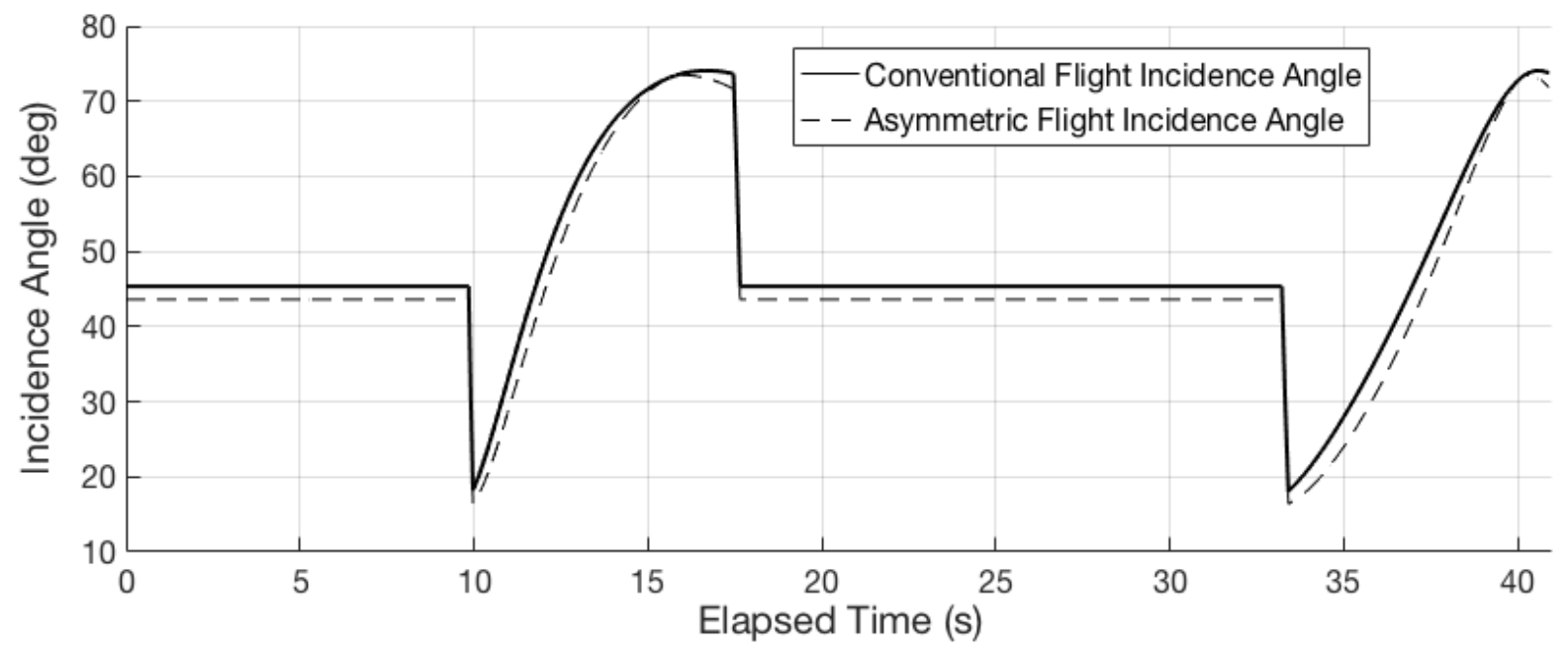

Figure A.8: Time history of incidence angle for a sample grid flight on the summer solstice in San Luis Obispo, CA.

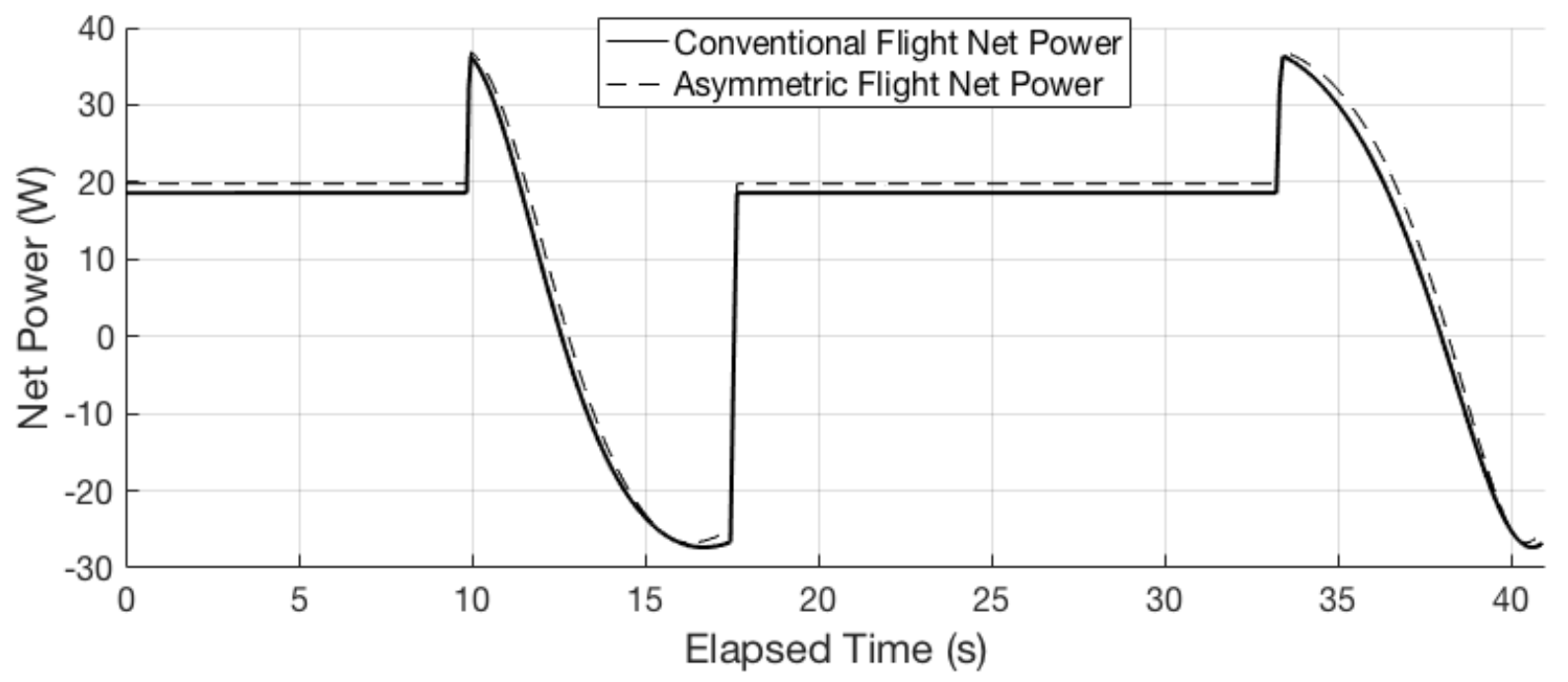

Figure A.9: Time history of net power for a sample grid flight on the summer solstice in San Luis Obispo, CA. 


\section{Appendix B}

\section{MATLAB CODE}

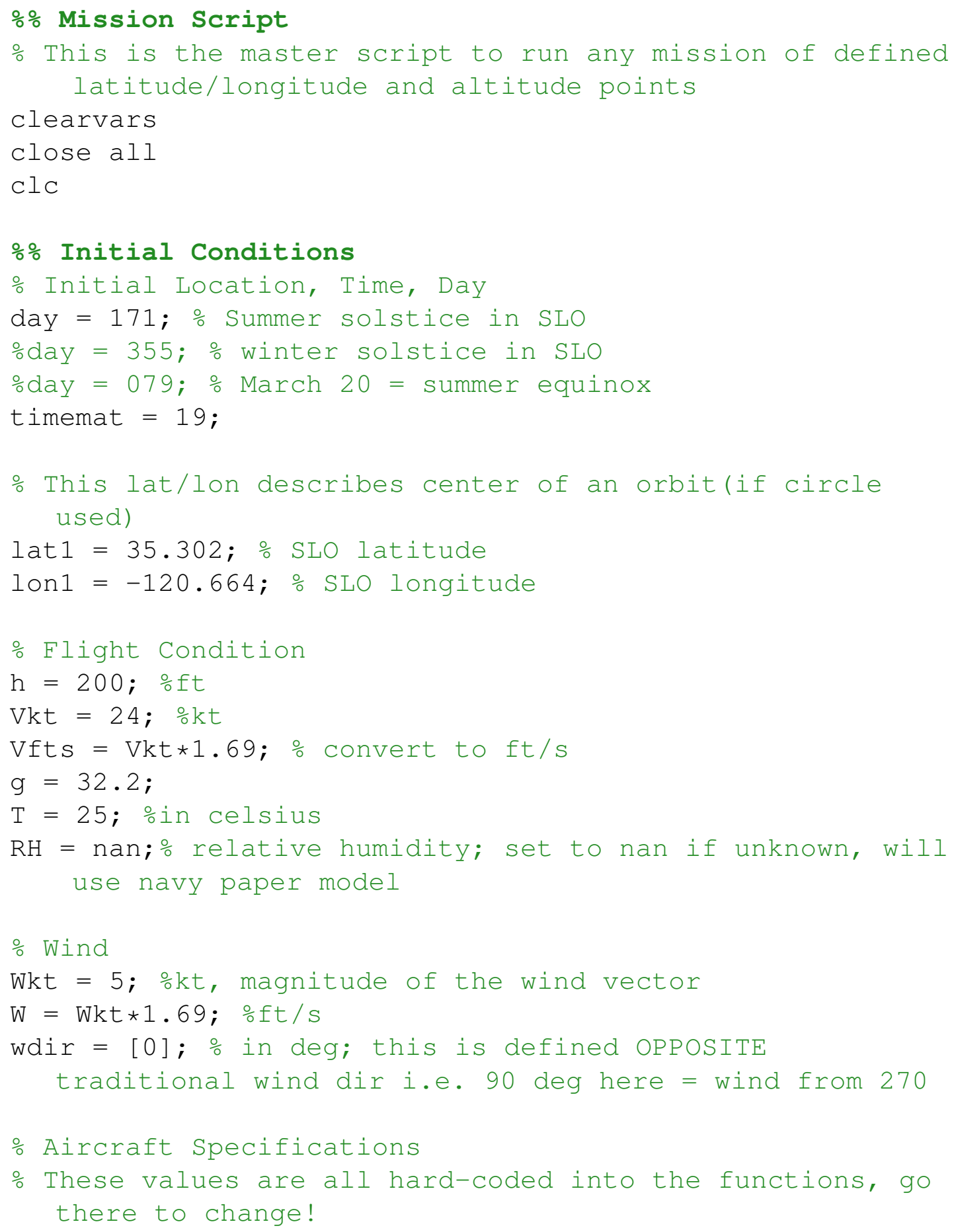




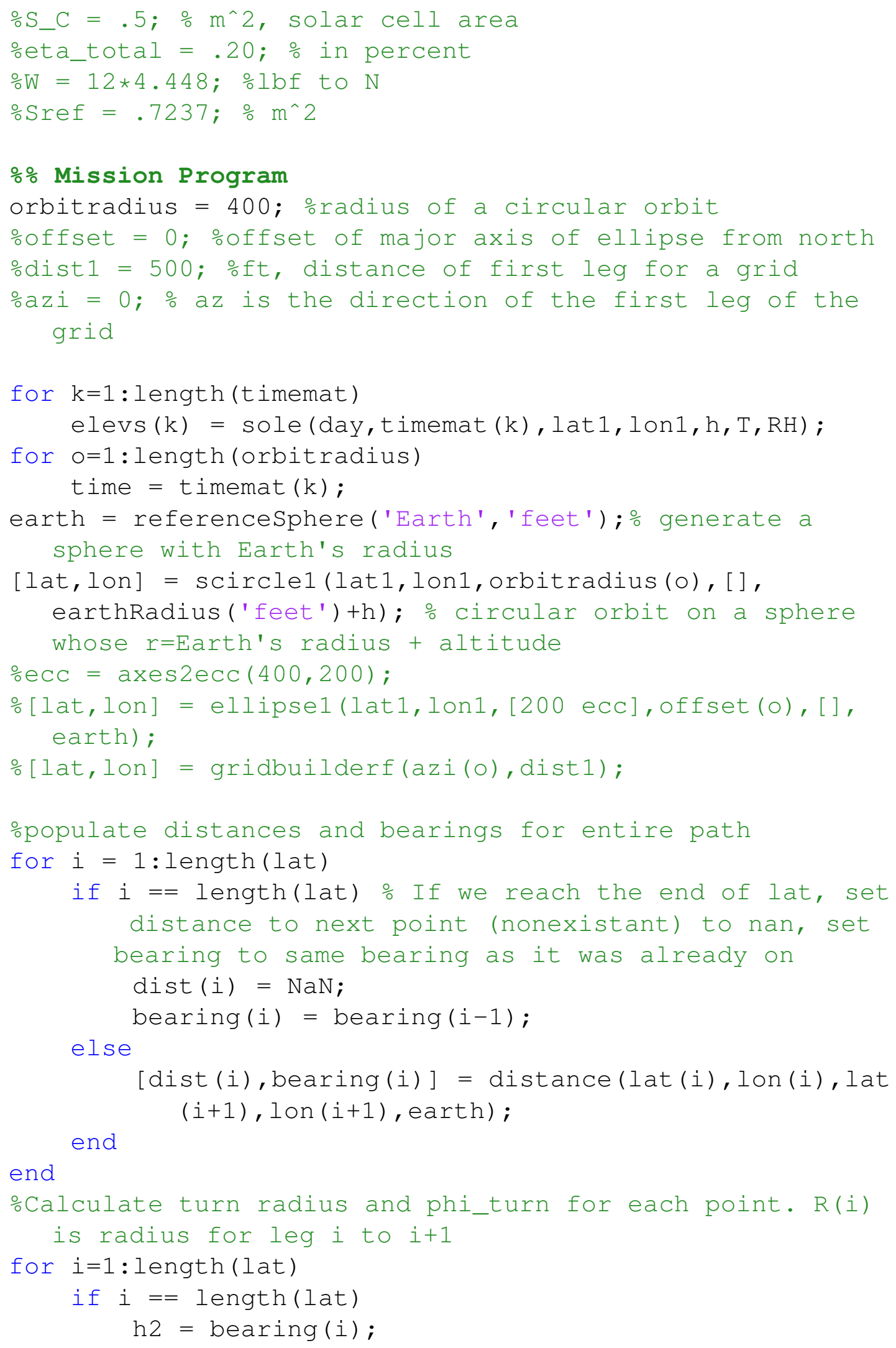




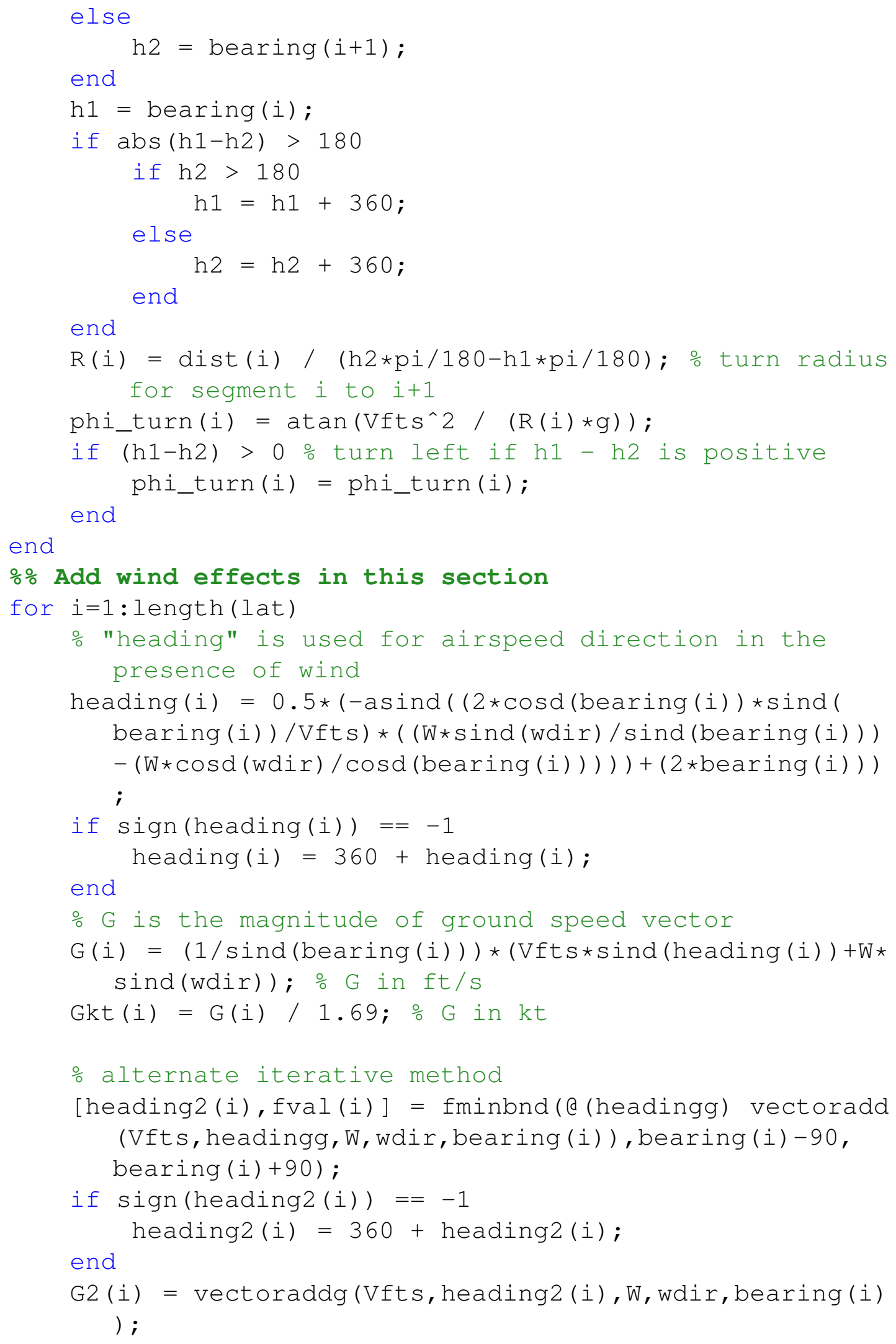




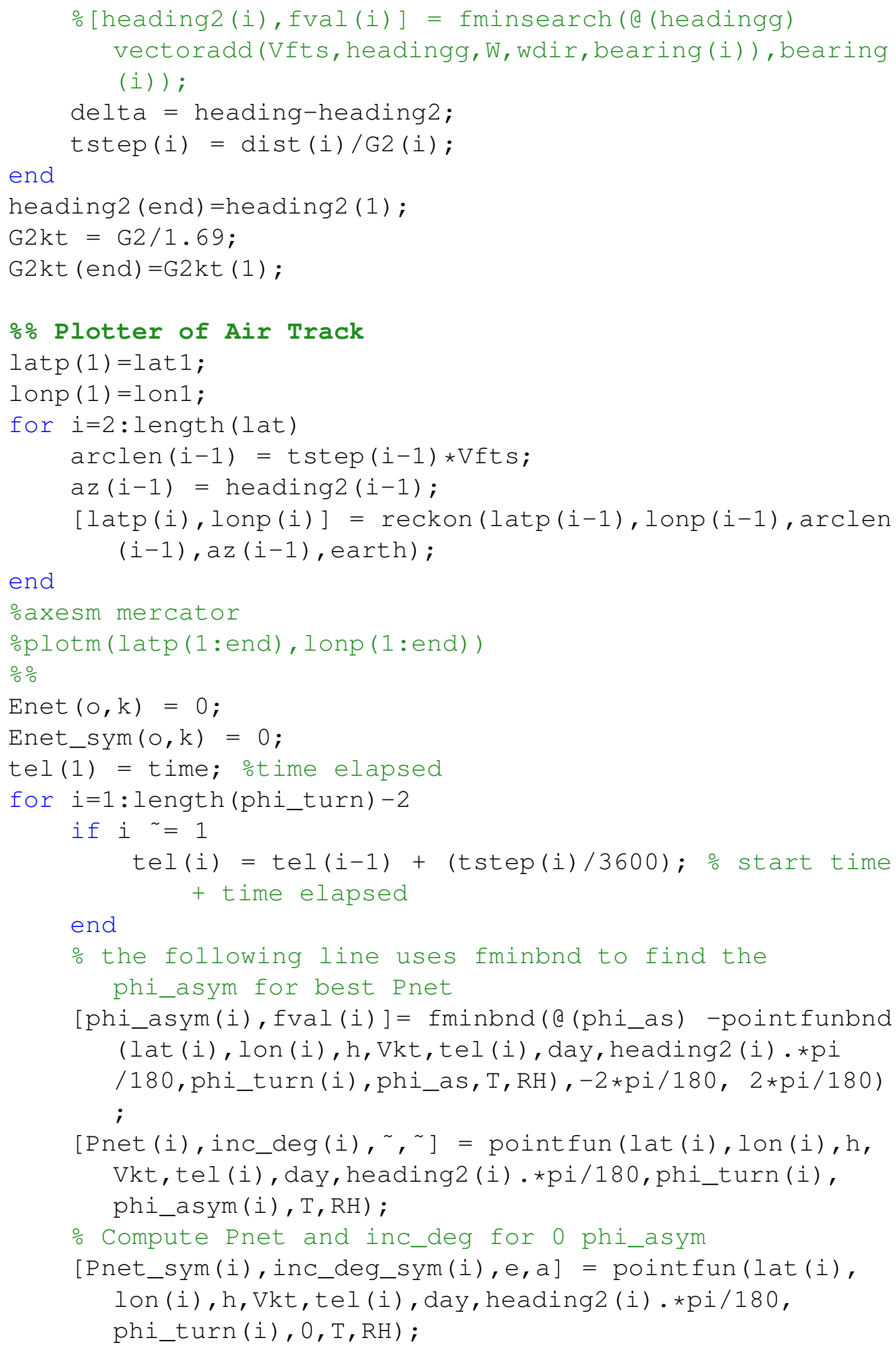




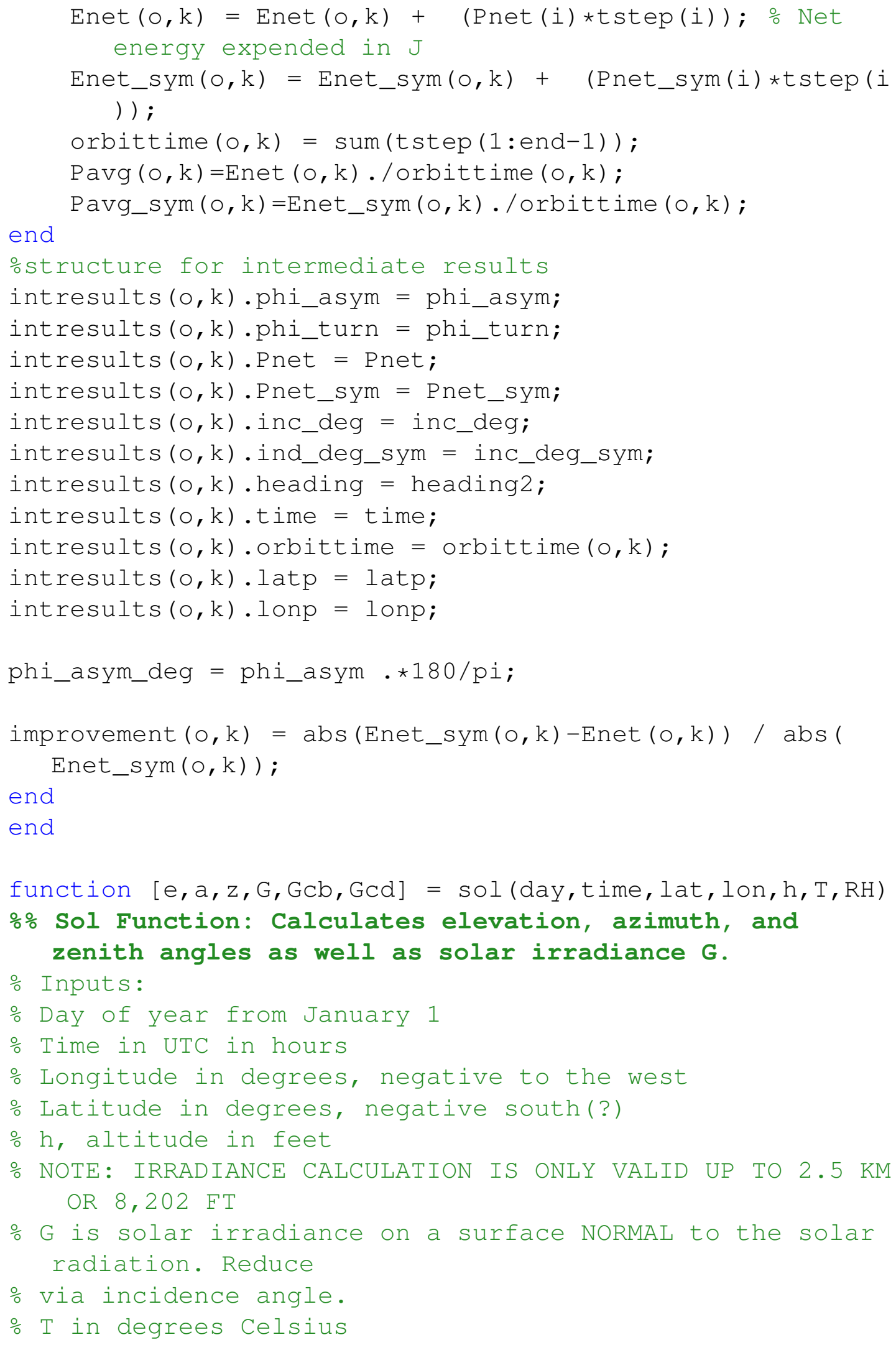




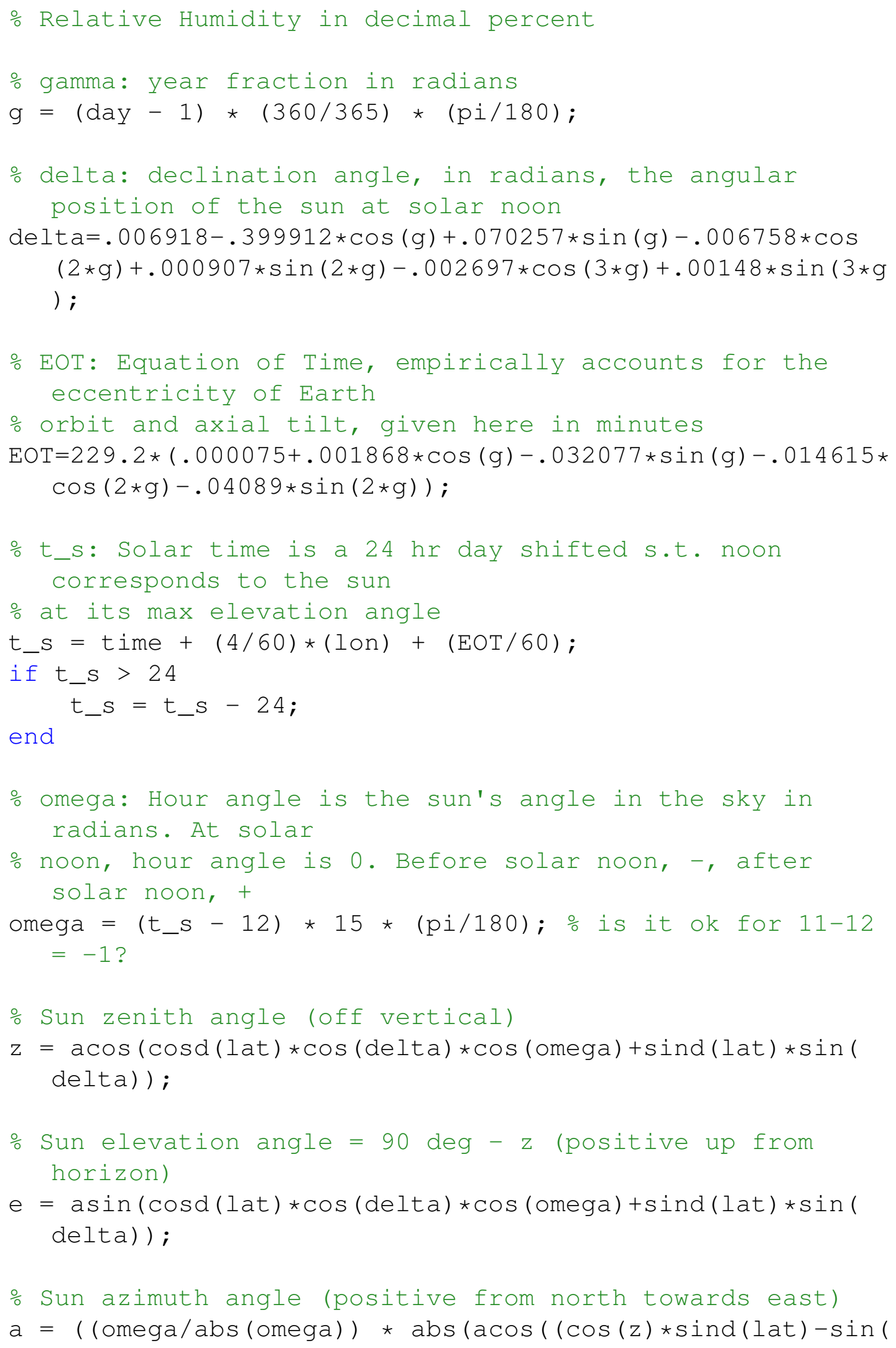




$$
\operatorname{delta})) /(\sin (z) \star \cos ((\operatorname{lat})))))+\mathrm{pi} \text {; }
$$

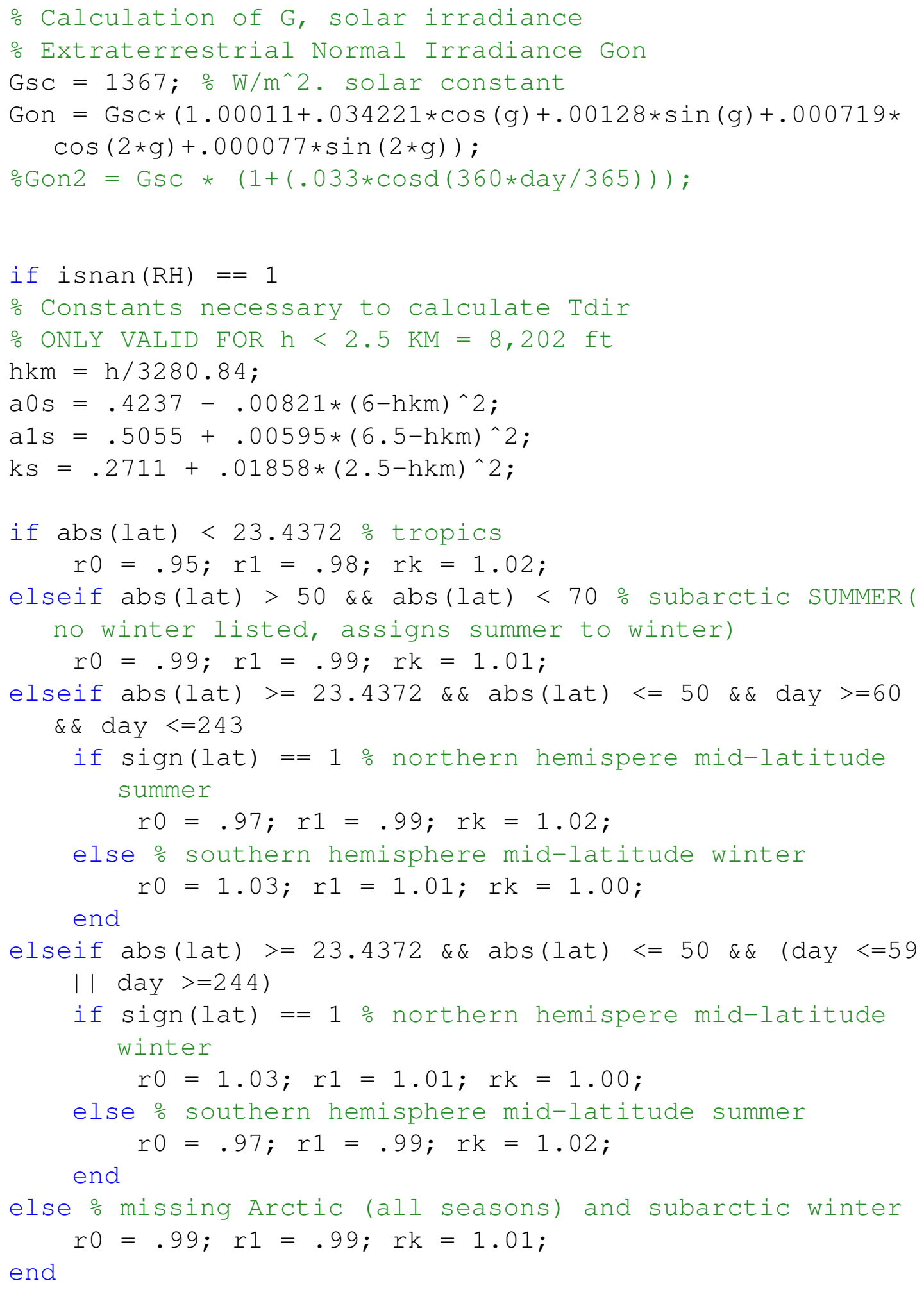




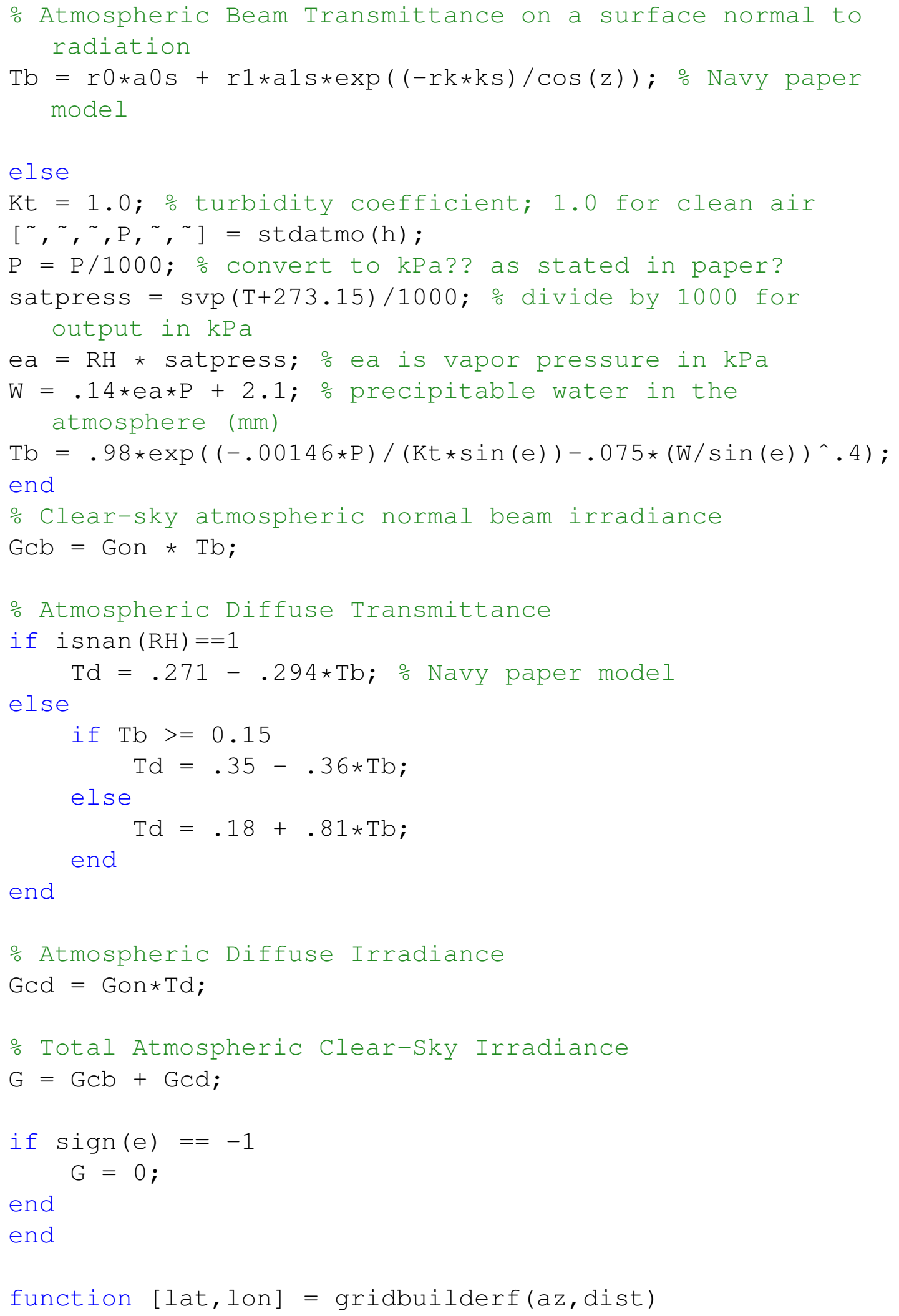




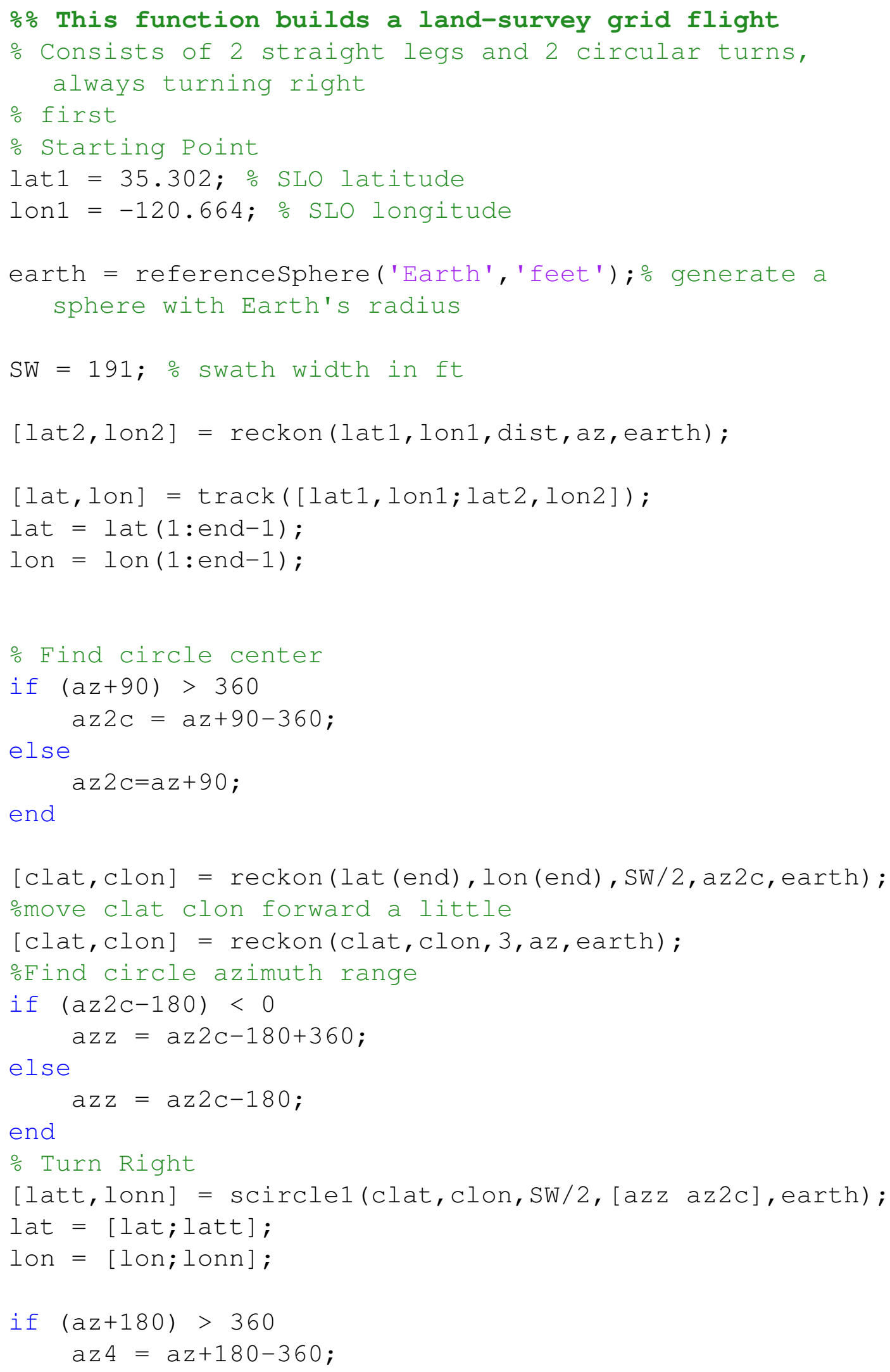




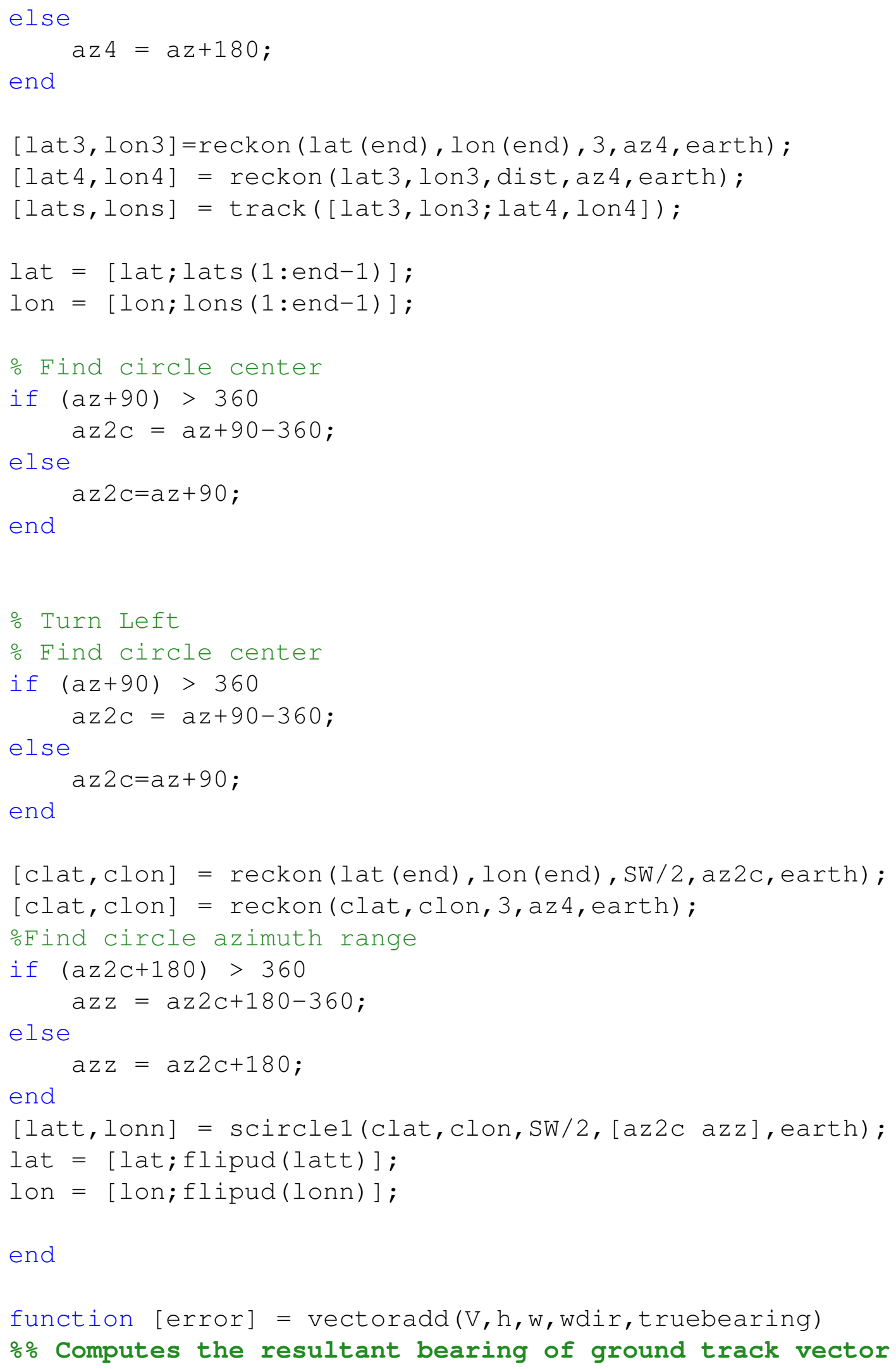




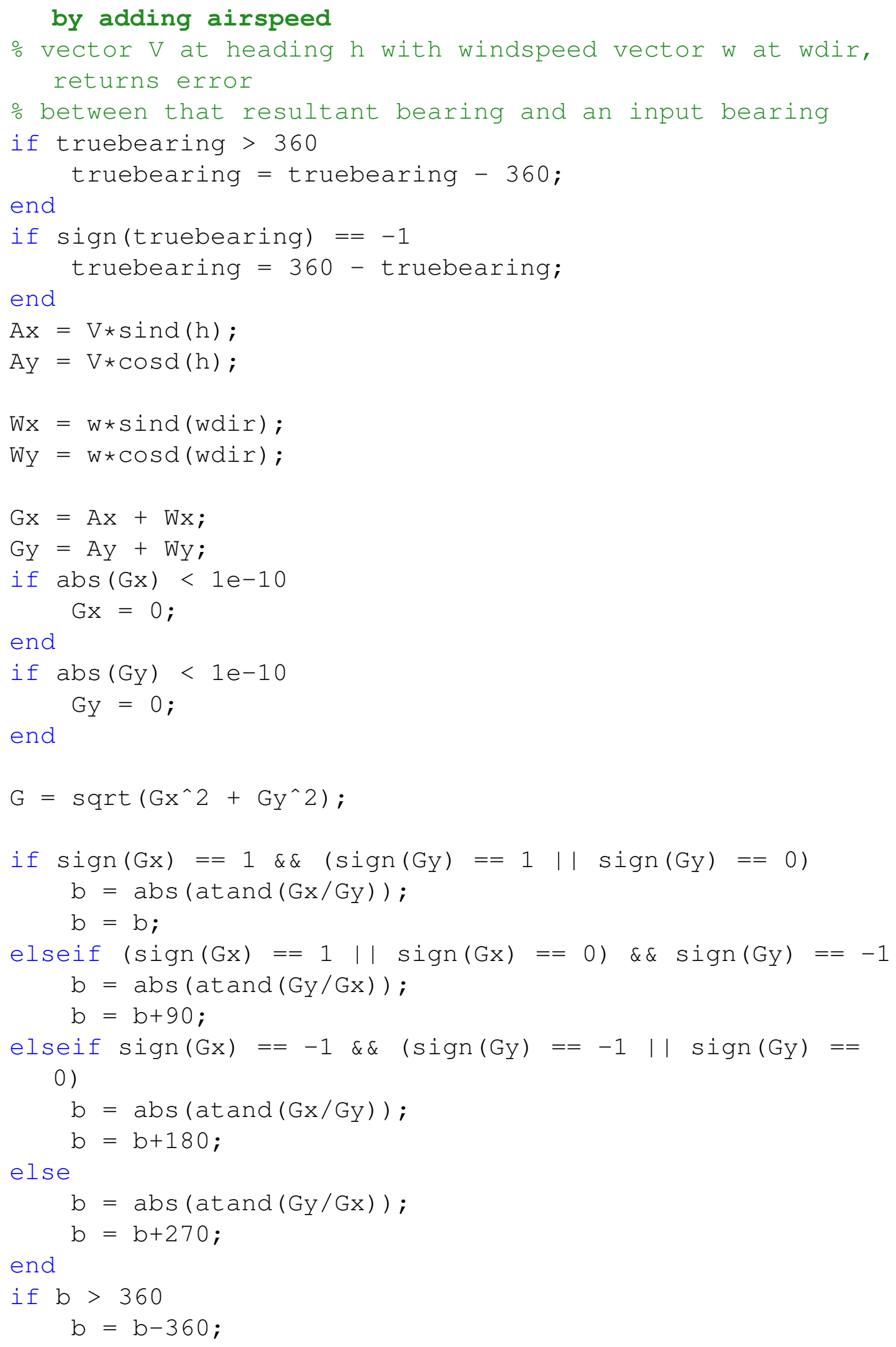




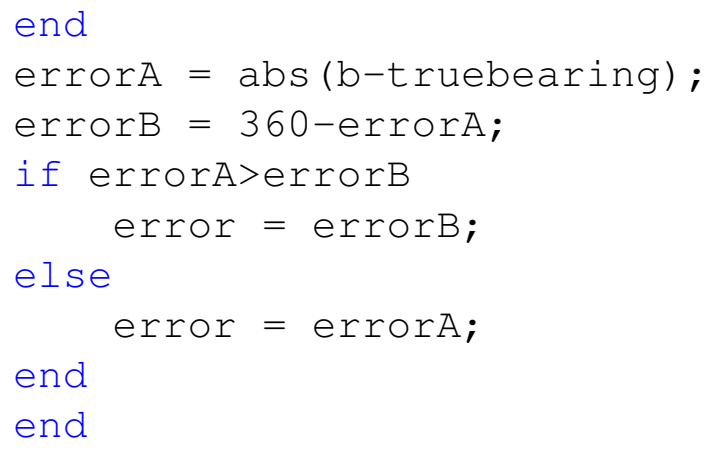




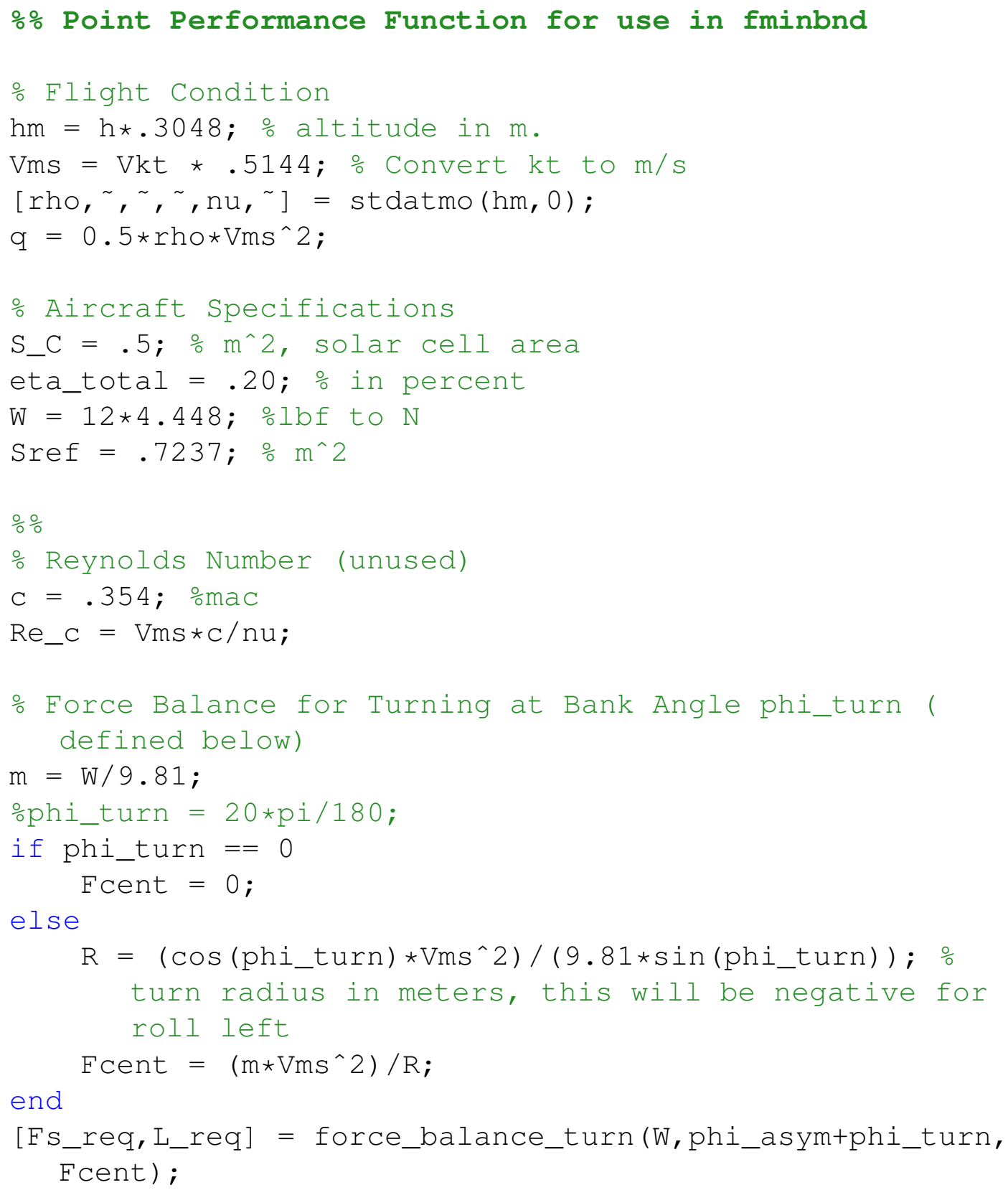




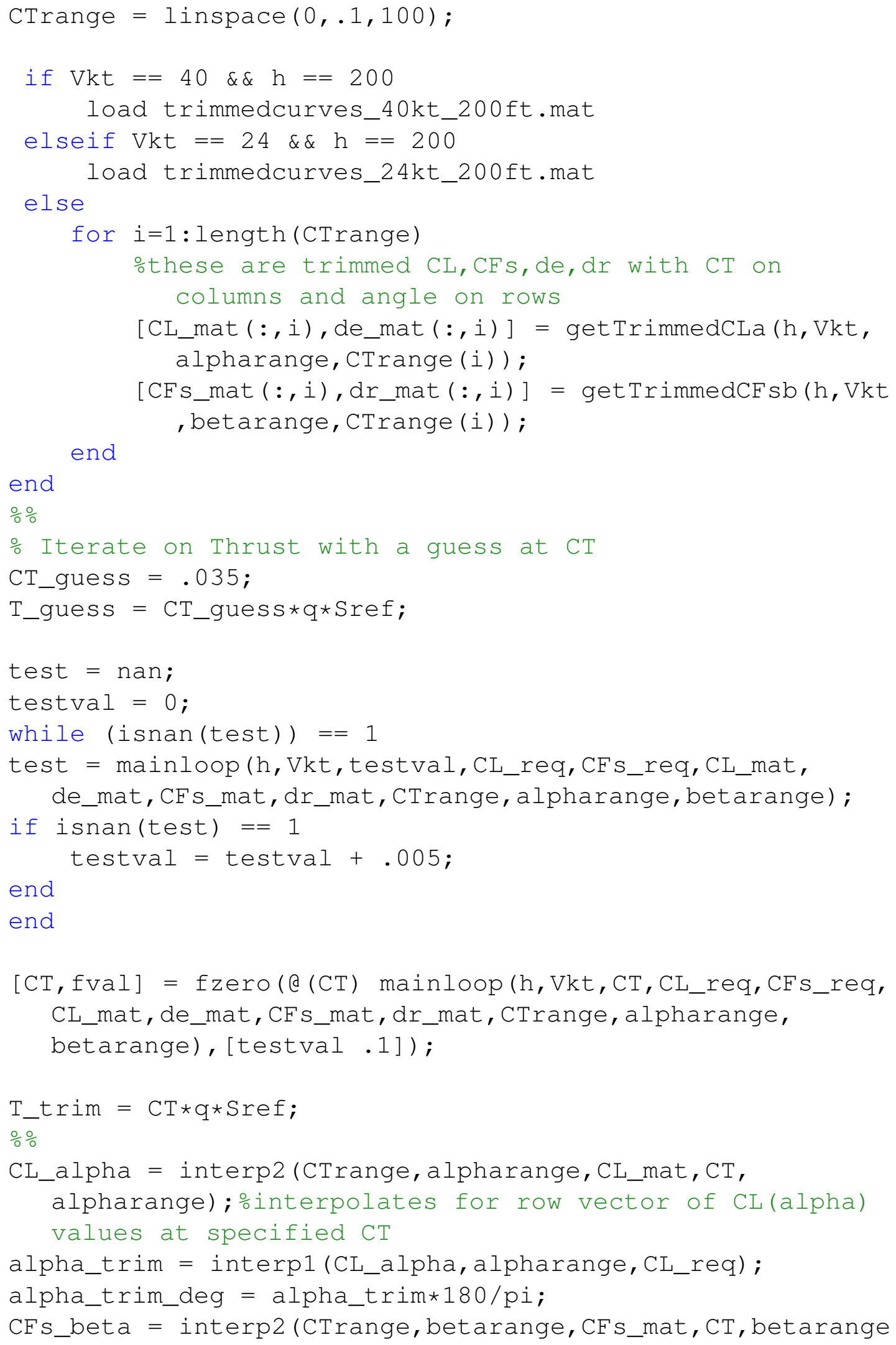


);

beta_trim = interp1 (CFs_beta, betarange,CFs_req); $\circ$ deleted pchip here to disable extrapolation

if abs (beta_trim) $<1 e-14$

beta_trim=0;

end

beta_trim_deg $=$ beta_trim * 180/pi;

de $=$ interpl (CL_alpha, de_mat $(:, 1)$, CL_req);

de_deg $=$ de $\star 180 / \mathrm{pi}$;

$d r=$ interp1 (CFs_beta,dr_mat $\left.(:, 1), C F s \_r e q\right) ; \%$ deleted

pchip here to disable extrapolation

if $\operatorname{abs}(\mathrm{dr})<1 e-13$

$d r=0$;

end

dr_deg $=\mathrm{dr} \star 180 / \mathrm{pi}$;

\% Pull drag values with final alpha, beta, de, dr

$\left[C D \_t o t, C D \_w, C D \_b, C D \_h t, C D \_v t\right]=$ dragbuild $(h, V k t$,

beta_trim, alpha_trim, de, dr);

P_required $=\mathrm{CD} \_t$ ot $*\left(.5 * r h o * \mathrm{Vms}^{\wedge} 2\right) *$ Sref $* \mathrm{Vms}$; $\%$ Preq in Watts

\section{$\circ$ Sun}

$[e, a, \sim, G]=\operatorname{sol}($ day, time, lat, lon, h, $\mathrm{T}, \mathrm{RH})$;

$\circ \mathrm{e}=45 * \mathrm{pi} / 180$;

$\circ \mathrm{a}=90 * \mathrm{pi} / 180$;

o Note: if inc is 90, it is likely that the sun is behind the solar panel

[inc] = incidence (e, a,phi_asym+phi_turn,alpha_trim,psi+ beta_trim); $\%$ theta must $=$ alpha in this model

inc_deg $=$ inc $\star 180 / p i$;

P_available $=G$ * cos $($ inc $) * S \_C *$ eta_total; $\%$ in Watts

응 Net Power

Pnet $=$ P_available - P_required;

end

function [Pnet, inc_deg, e, a, P_available,P_required] = pointfun(lat, lon, h, Vkt, time, day,psi,phi_turn,phi_asym, $\mathrm{T}, \mathrm{RH}$ )

응 Point Performance Function

o Input lat/lon in degrees, $h$ in ft, time in hours, heading(psi) in radians 


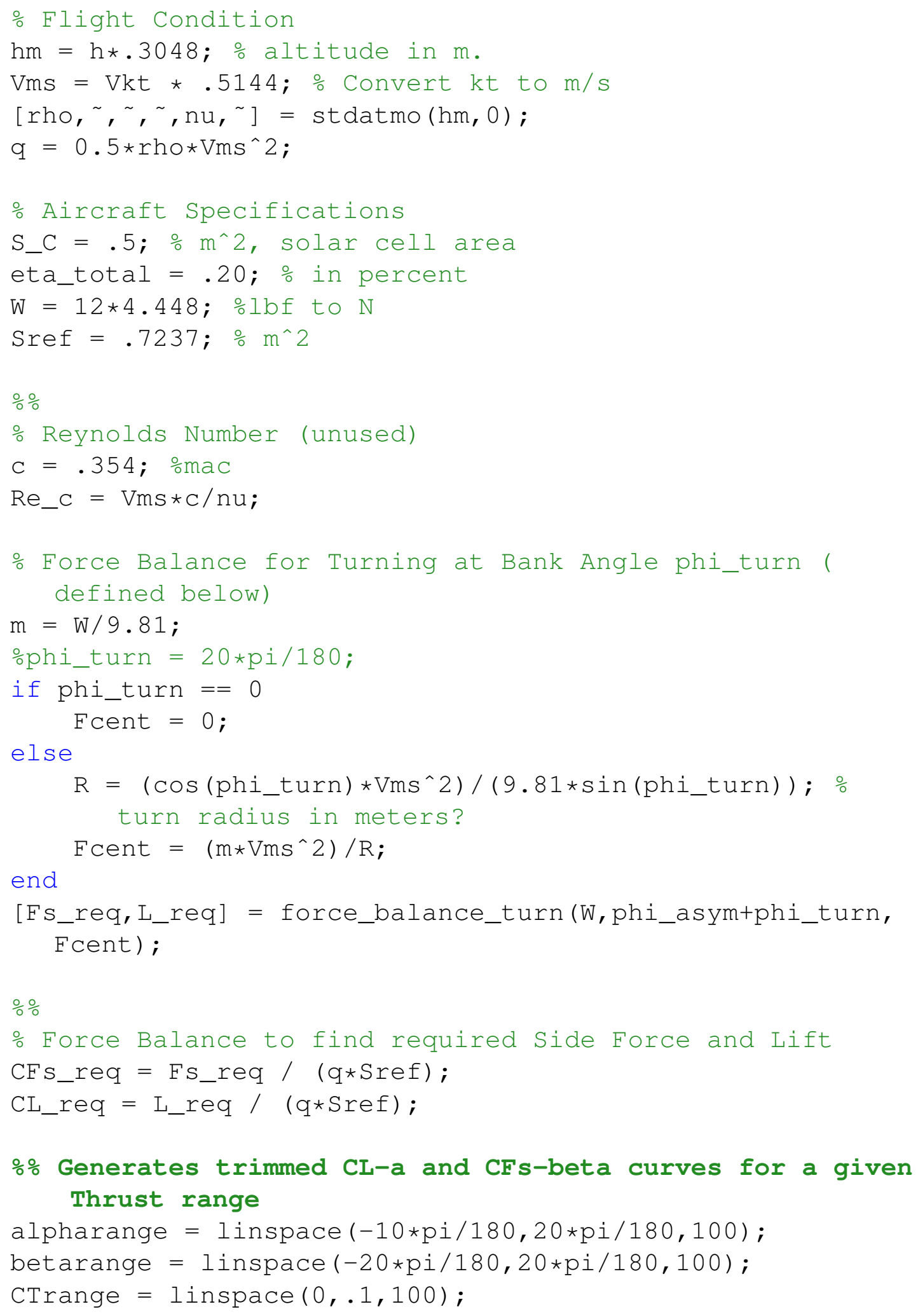




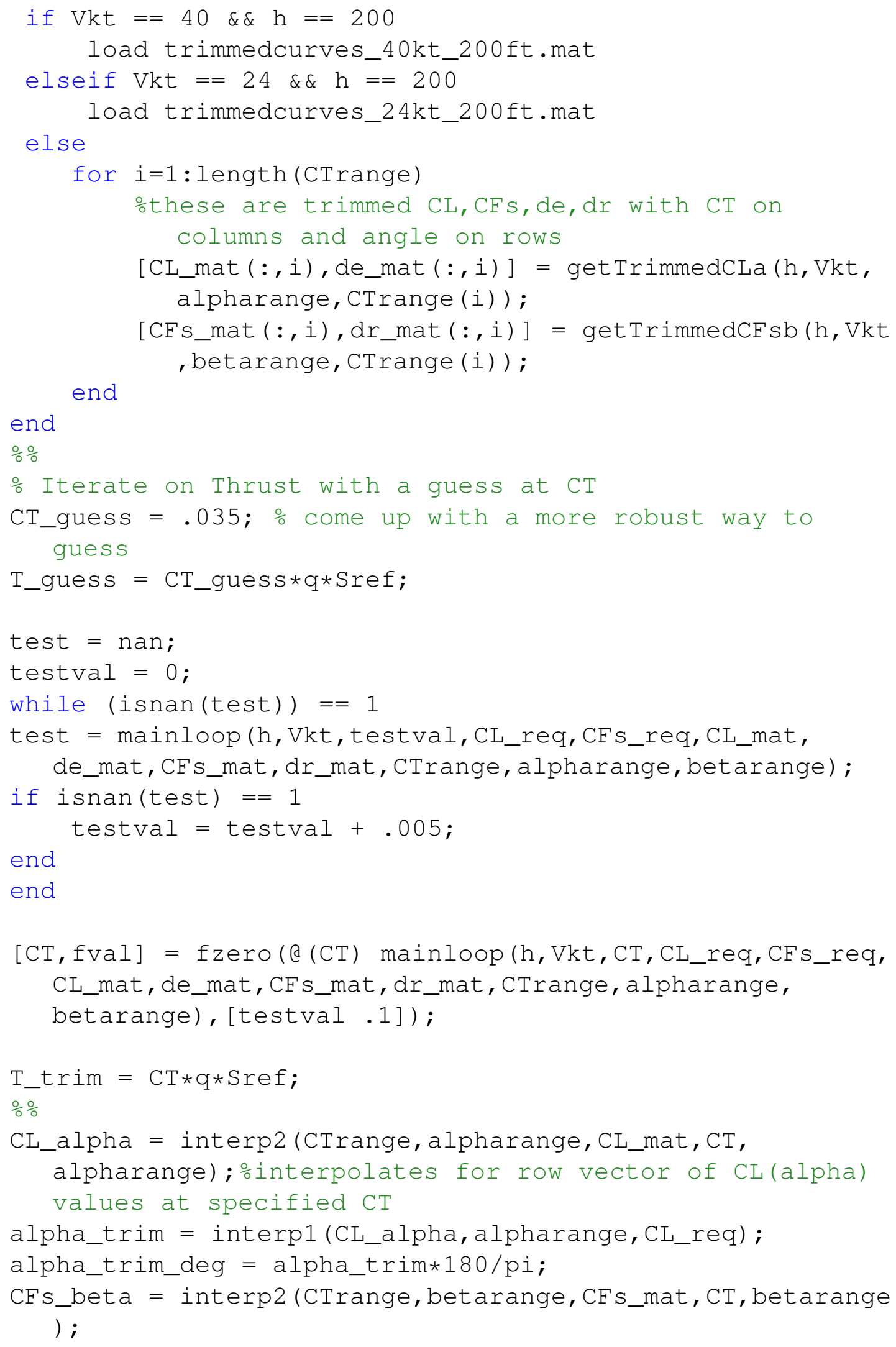




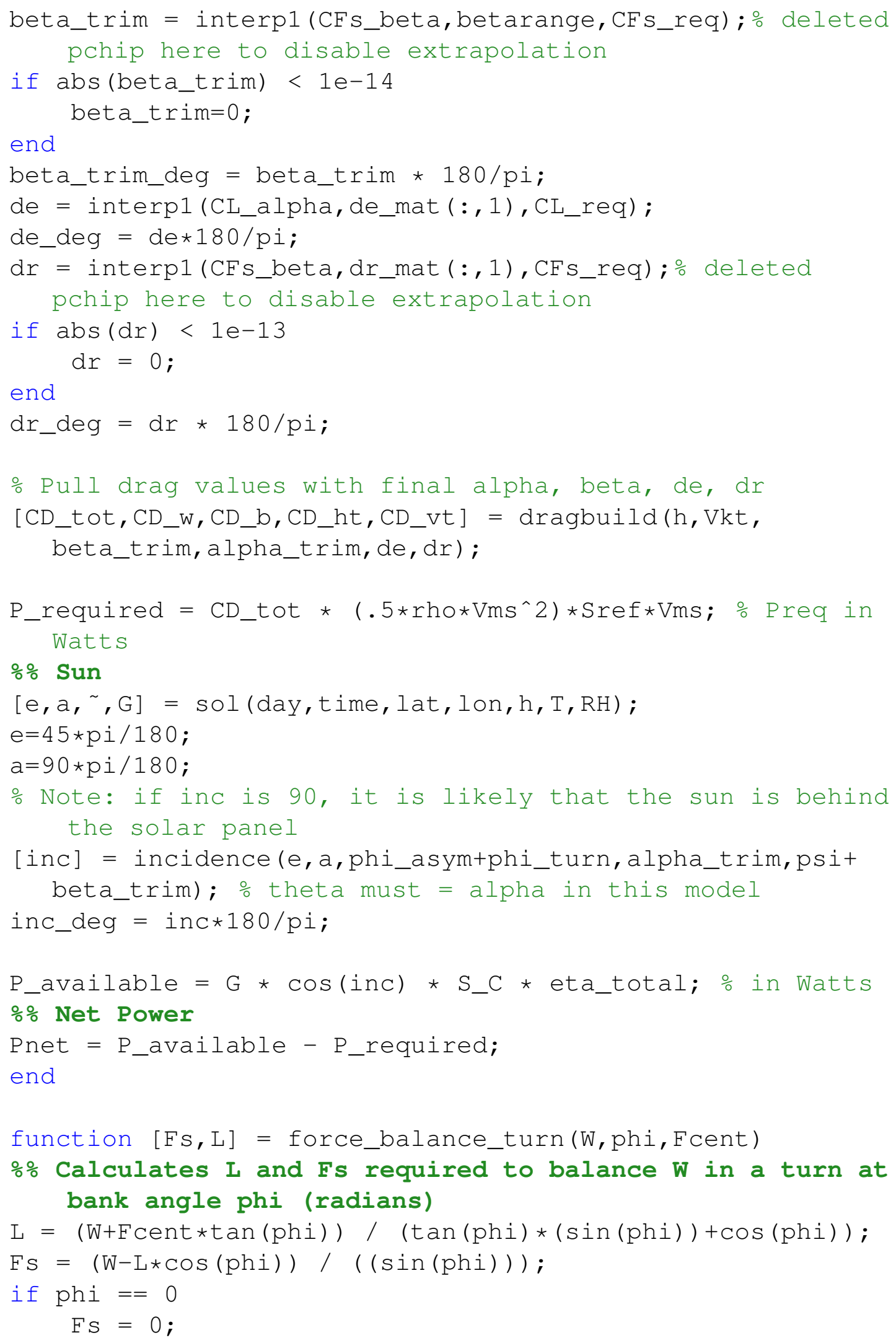




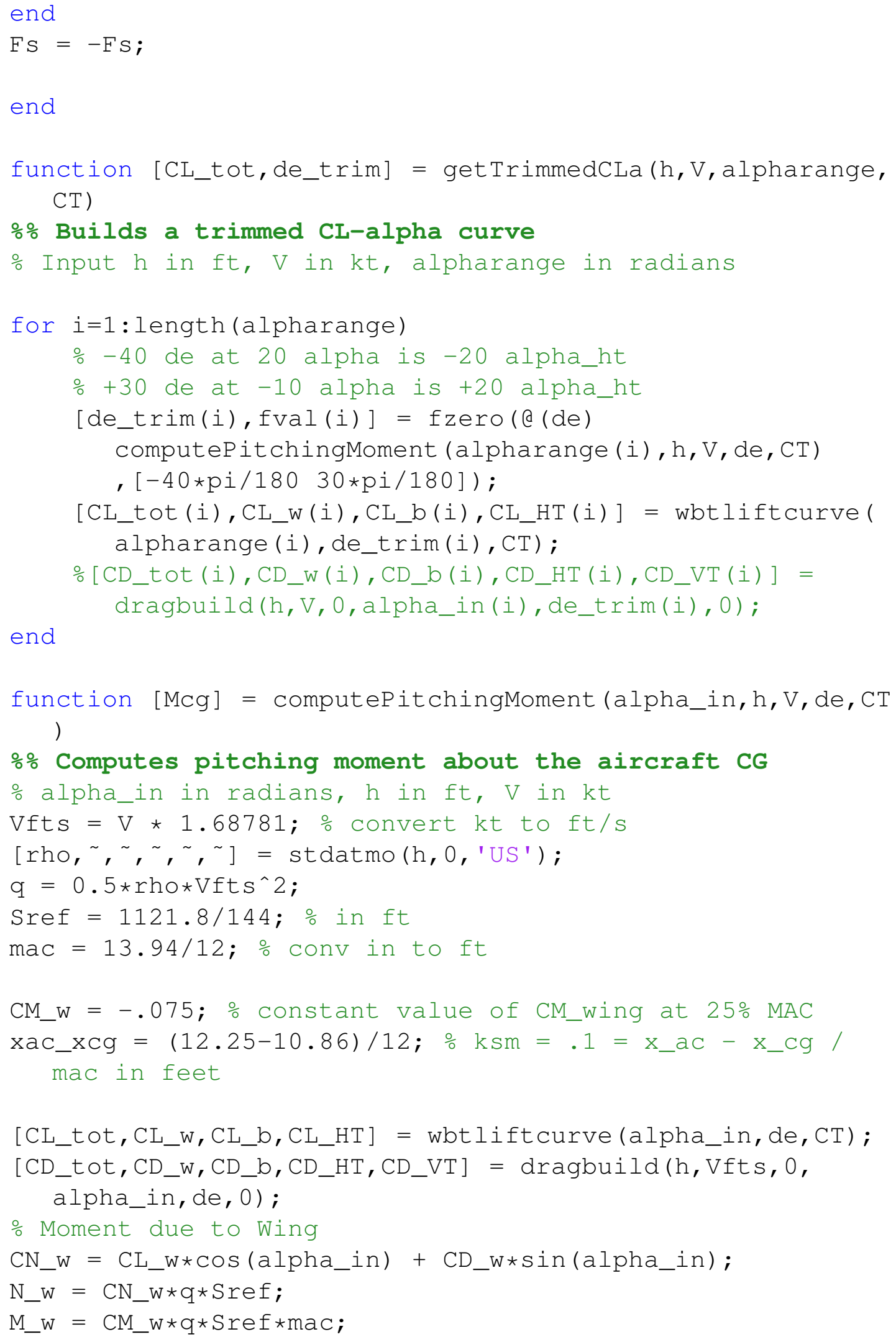




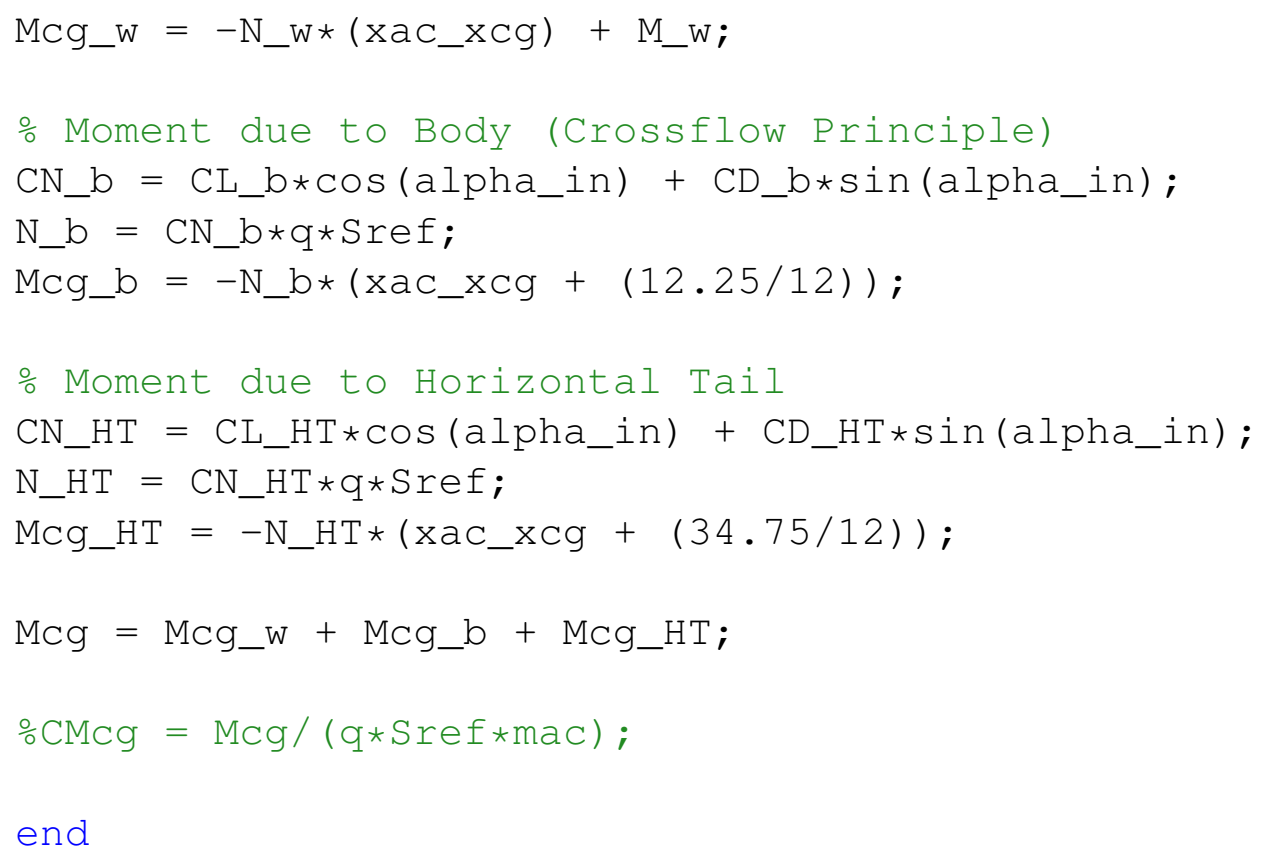




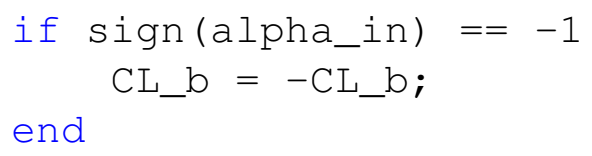




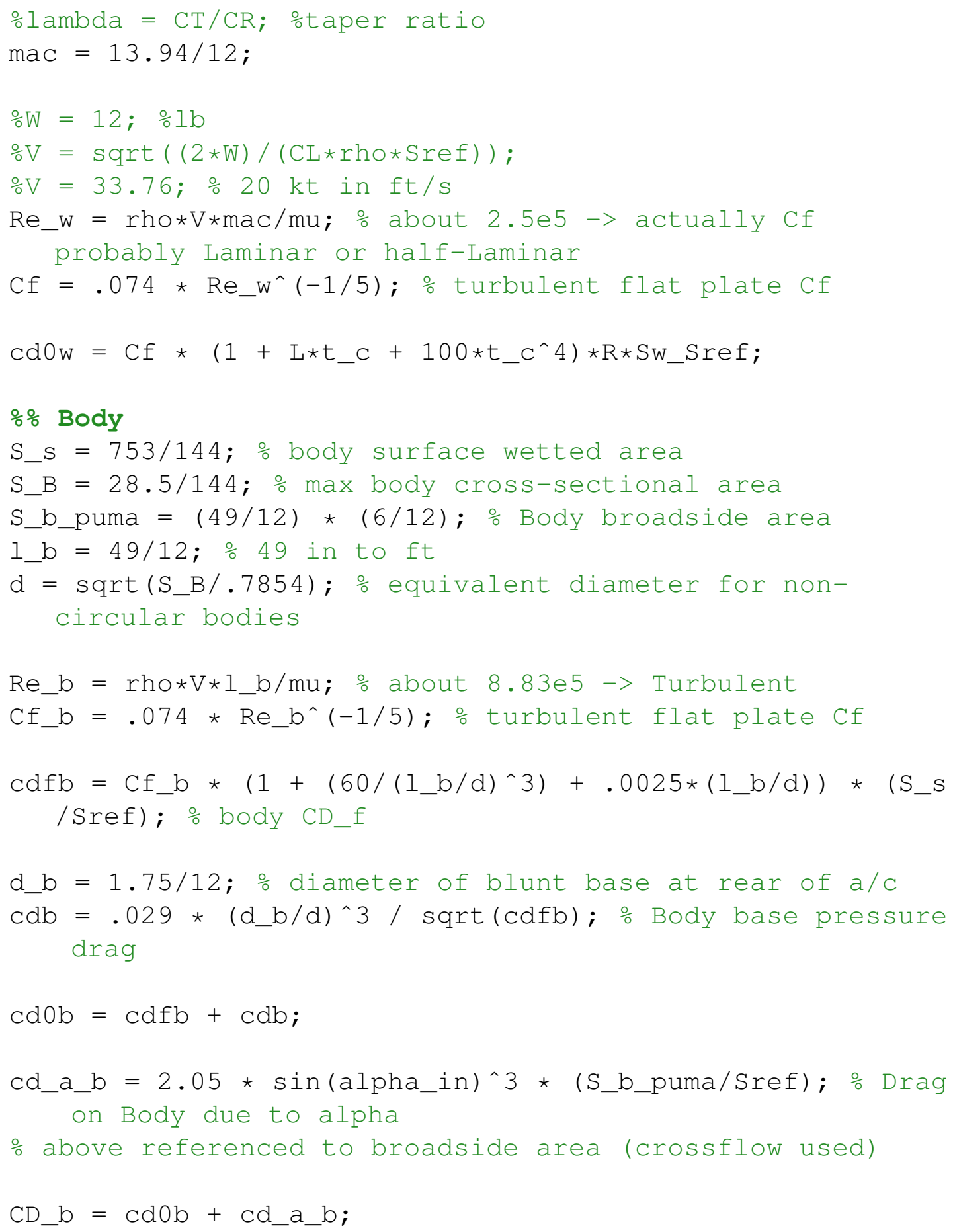




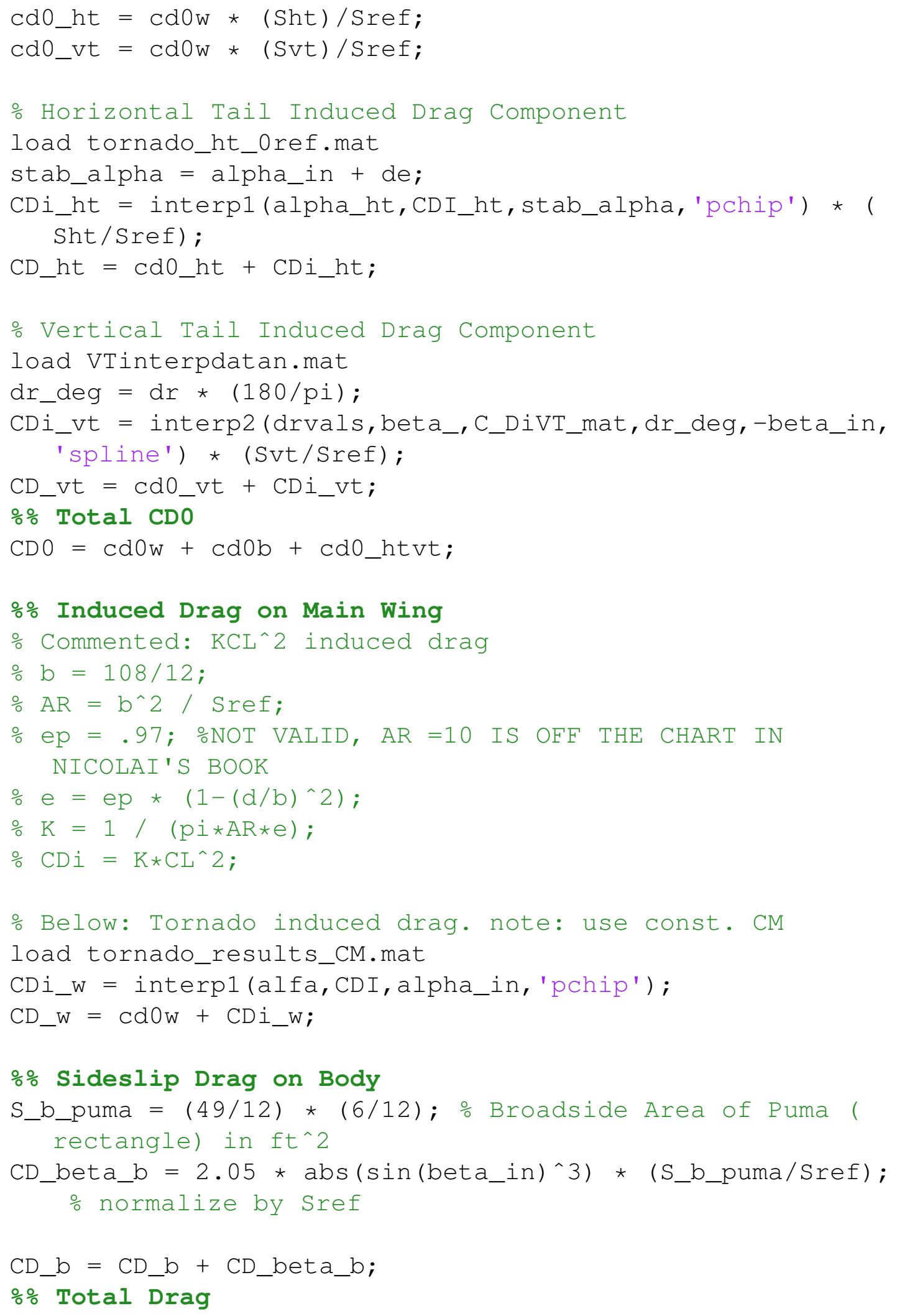




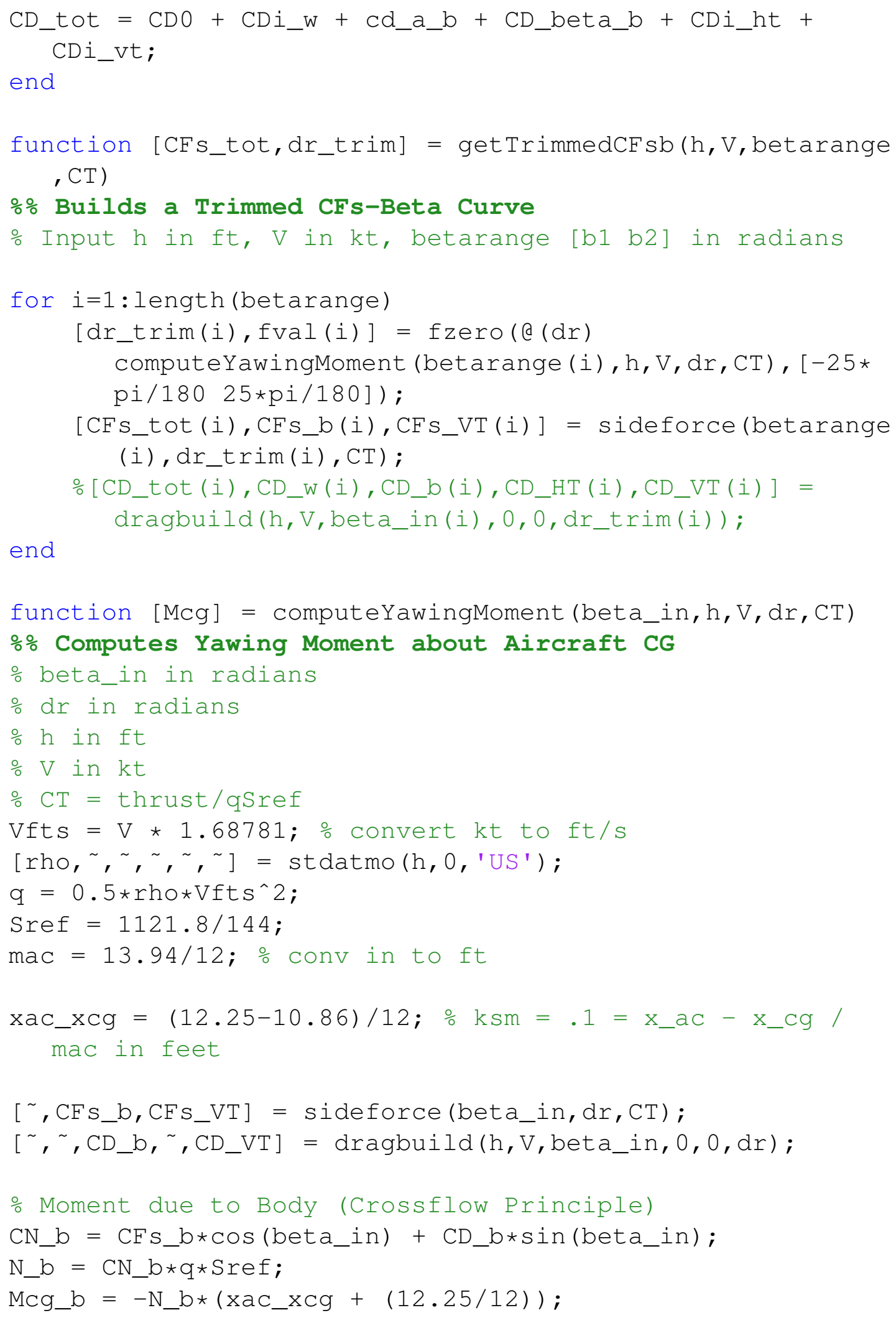




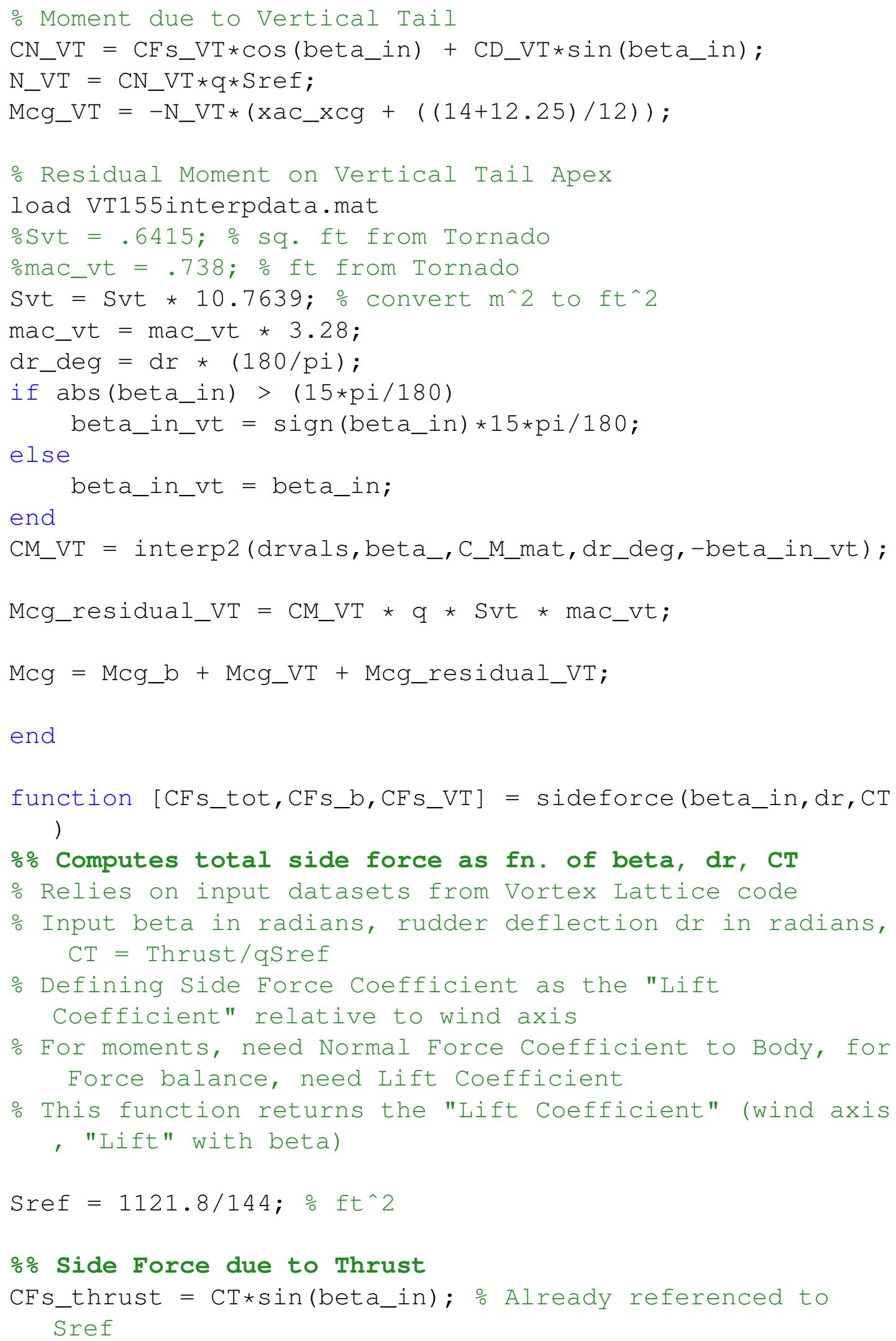




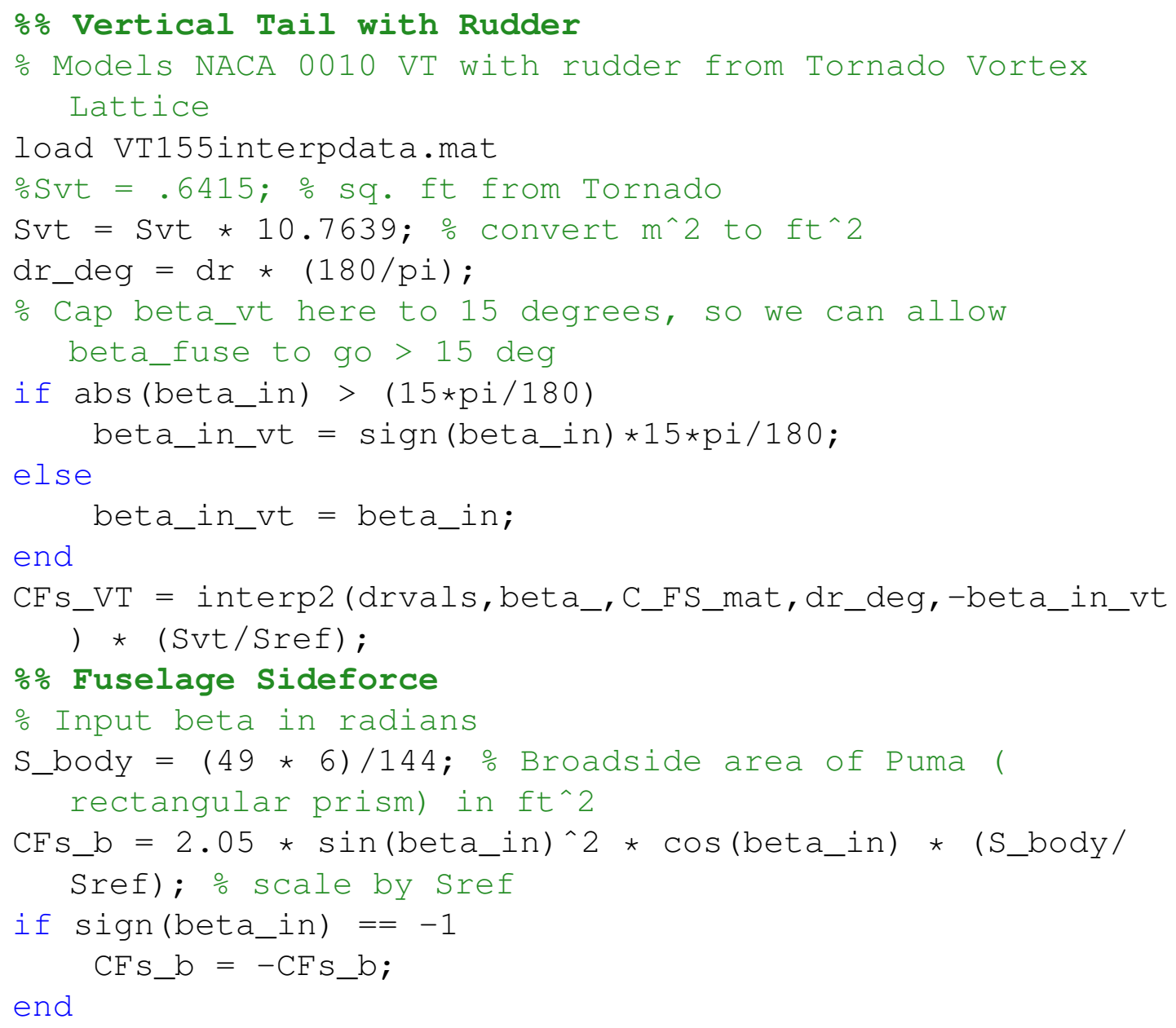




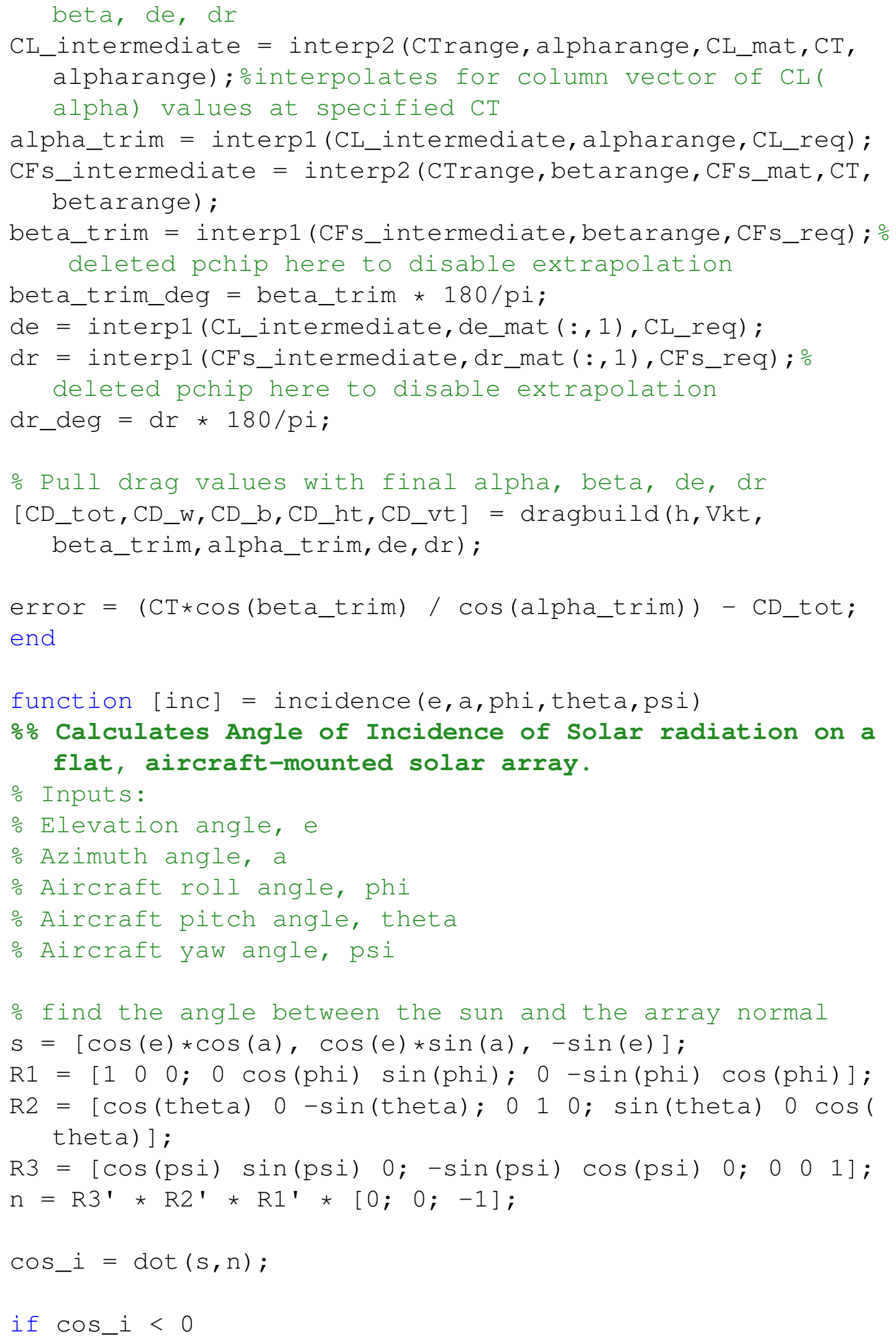




$$
\begin{aligned}
& \text { cos_i }=0 ; \\
& \text { end } \\
& \text { inc }=\text { acos (cos_i }) \text {; } \\
& \text { end }
\end{aligned}
$$

